\title{
A LAW OF LARGE NUMBERS FOR FAST PRICE ADJUSTMENT
}

\author{
H. JEROME KEISLER
}

\begin{abstract}
The purpose of this paper is to prove a law of large numbers for certain Markov processes involving large sets of weakly interacting particles. Consider a large finite set $A$ of "particles" which move about in $m$-dimensional Euclidean space $R^{m}$. The particles interact with each other indirectly by means of an auxiliary quantity $p$ in $d$-dimensional Euclidean space $R^{d}$. At each time $t$, a particle $a \in A$ is randomly selected and randomly jumps to a new location in $R^{m}$ with a distribution depending on $p$ and its old location. At the same time, the value of $p$ changes to a new value depending on these same arguments. The parameter $p$ moves by a small amount at each time but moves fast compared to the average position of the particles.

Under appropriate hypotheses on the rules of motion, we shall prove the following law of large numbers. For sufficiently large $A$, the value of $p$ will be close to its expected value with large probability, and the average position of the particles will be close to its expected value with large probability.

The work was motivated by the problem of modelling the adjustment of prices in mathematical economics, where the particles $a \in A$ are agents in an exchange economy, the position of $a$ at time $t$ is the commodity vector held by agent $a$ at time $t$, and $p$ is the price vector at time $t$.
\end{abstract}

\section{INTRODUCTION}

The purpose of this paper is to prove a law of large numbers for certain Markov processes involving large sets of weakly interacting particles. The work was motivated by the problem of modelling the adjustment of prices in mathematical economics, but the results are proved in a general mathematical context which is capable of other interpretations.

The economic problem is to describe the movement of prices toward an equilibrium price in an exchange economy, particularly after a sudden change in economic conditions. Intuitively, the price adjustment process should be very fast, so that few people will trade before the price gets close to the equilibrium value. The traditional model of this process, called tâtonnement, has a fictitious auctioneer. The auctioneer announces a price vector, then the traders announce their demands without actually trading, and then the auctioneer adjusts the price, continuing the process until an equilibrium is reached. The results in this paper give a more realistic probabilistic model where the auctioneer is replaced by a central warehouse and some trade occurs even when the price is out of equilibrium. The individuals who are able to respond quickly to a change in

Received by the editors July 13, 1987 and, in revised form, April 2, 1990.

1980 Mathematics Subject Classification (1985 Revision). Primary 03H10, 60B12, 60J10, 90A 16. 
the environment may reap large gains, but the information from these trades is used by the central warehouse to rapidly adjust the prices. The main result is a law of large numbers stating that with large probability the price path and the economic environment will be close to their expected values.

Consider a large finite set $A$ of "particles" which move about in $m$-dimensional Euclidean space $R^{m}$. The particles interact with each other indirectly by means of an auxiliary quantity $p$ in $d$-dimensional Euclidean space $R^{d}$, as follows. Time is divided into discrete intervals of length $\Delta t, T=\{n \cdot \Delta t: n \in$ $\mathbf{N}\}$. Each particle $a \in A$ has an initial position $X_{0}(a) \in R^{m}$ and $p$ has an initial value $p_{0} \in R^{d}$. At each time $t \in T$, two independent random choices are made. A particle $a=\omega_{A}(t) \in A$ and an element $\omega_{\Gamma}(t)$ of another finite set $\Gamma$ are selected, with all selections independent over time and of the same probability. (Think of a particle being chosen and a \#( $\Gamma)$-sided die being thrown.) The particle $a=\omega_{A}(t)$ which was selected jumps to a new location in $R^{m}$ depending on its old location, the element $\omega_{\Gamma}(t)$, and the value of $p$. At the same time, the value of $p$ changes to a new value depending on these same arguments. The other particles do not move at time $t$. For convenience we set $\Delta t$ equal to the probability that a single particle is chosen at time $t$.

At each time $t$, the parameter $p$ moves by a distance which is large compared to $1 / \#(A)$, where $\#(A)$ is the cardinality of $A$, but small compared to 1 . Thus $p(\omega, t)$ moves by a small amount at each time but moves fast compared to the average position of the particles. The speed of motion of $p$ will depend on a parameter $\delta$ of the form $\delta=\#(A)^{-l}$, where $0<l<1$. The value $p(\omega, t)$ and the position $X(\omega, a, t)$ of particle $a$ at time $t$ are given by the following difference equations involving two functions $f$ and $g$ and the number $\delta$ :

$$
\begin{array}{cc}
\Delta p(\omega, t)=f\left(\omega_{\Gamma}(t), X(\omega, a, t), p(\omega, t)\right) \cdot \delta, & \text { where } a=\omega_{A}(t), \\
\Delta X(\omega, a, t)= \begin{cases}0 & \text { if } a \neq \omega_{A}(t), \\
g\left(\omega_{\Gamma}(t), X(\omega, a, t), p(\omega, t)\right) & \text { if } a=\omega_{A}(t) .\end{cases}
\end{array}
$$

In the original motivating problem in economics, the particles $a \in A$ are agents in an exchange economy, the position $X(\omega, a, t)$ is the commodity vector held by agent $a$ at time $t$, and the value $p(\omega, t)$ is the price vector at time $t$. At each time $t$, the agent $a=\omega_{A}(t)$ selected at time $t$ makes an exchange with a "central warehouse" and obtains a new commodity vector. On the basis of this exchange, the price vector $p(\omega, t)$ is adjusted by an amount of order $\delta$. The function $f$ is the excess demand function, and the function $g$ and parameter $\delta$ determine a rule for adjusting the price. The set $\Gamma$ allows for additional random arguments which can affect the exchange at time $t$.

We shall prove the following law of large numbers. Assume that the functions $f$ and $g$ are uniformly Lipschitz in $x$ and $p$ and linearly bounded in $x$, and $f$ is exponentially stable (in a sense described in $\S 1$ ). Let $l$ be a constant between 0 and 1 . Let the set $A$ grow but keep fixed $l$, the functions $f$ and $g$, a bound for the initial value $p(0)$ and initial positions $X(a, 0)$, and the finite set $\Gamma$. Then for sufficiently large $A$ and for $\delta=\#(A)^{-l}$, the value of $p(\omega, t)$ will be close to its expected value with large probability, and for each linearly bounded Lipschitz function $\varphi$ on $R^{m}$, the average value of $\varphi(X(\omega, a, t))$ over $A$ will be close to its expected value with large probability.

To handle the positions $X(\omega, a, t)$ for all $a \in A$ at once, the measure $b_{X}$ 
on $R^{m}$ determined by a function $X$ from $A$ into $R^{m}$ is treated as an element of a certain Banach space $\mathbf{B}$, which we call the state space. We then obtain a pair $p(\omega, t) \in R^{d}$ and $b(\omega, t) \in \mathbf{B}$, where $p(\omega, t)$ moves fast and $b(\omega, t)$ moves slowly with $t . b(\omega, t)$ is the distribution of the positions of $a \in A$ at time $t$. Our results will show that for large $A, b(\omega, t)$ will be close to its expected value in the state space $\mathbf{B}$ with large probability.

In order to simplify the bookkeeping and to describe the behavior of the paths $p(\omega, t)$ and $b(\omega, t)$, we shall pass to a nonstandard setting. An important tool in this setting is the Loeb measure, which is a countably additive probability measure generated from the counting measure on a hyperfinite set in a natural way. Our law of large numbers (Theorems 5.4-5.6) then says that for a hyperfinite set $A$ of particles, $p(\omega, t)$ and $b(\omega, t)$ will be infinitely close to their expected values with probability one in the Loeb measure. The parameter $\delta=\#(A)^{-l}$ will be infinitesimal. A complementary result (Theorem 5.7) describes the expected paths of $p(\omega, t)$ and $b(\omega, t)$ in terms of a system of differential equations. For infinitesimal times $t \in T$, the expected value $p(\omega, t)$ will be infinitely close to the solution of a standard differential equation speeded up by the infinite factor $1 / \delta$. Moreover, the expected value of $b(\omega, t)$ will be infinitely close to the solution of a standard differential equation in the state space $\mathbf{B}$, and for noninfinitesimal times $t$ the expected value of $p(\omega, t)$ will be infinitely close to a continuous function of the expected value of $b(\omega, t)$.

In $\S 1$ we establish an inequality on the growth of solutions of difference equations of a type which will arise in the body of the paper. These difference equations have a fast and slow component and small persistent disturbances. In $\S 2$ we define the central notion of an exchange process, introduce the state space, and show that certain auxiliary functions on the state space are well behaved. We then reformulate the original problem in terms of stochastic difference equations on the state space. In $\S 3$ we consider differential equations for the expected price and commodity distribution and prove that the solution exists. $\S 4$ contains the heart of the proof and makes use of nonstandard methods. The main results of this paper are in $\S 5$. Our law of large numbers is stated first in nonstandard terms as Theorem 5.4, and then in an equivalent standard form as Theorem 5.5.

Throughout this paper we shall use words from economics in our mathematical definitions for their mnemonic value, even though the paper is completely independent of notions from mathematical economics. Thus $A$ will be called the set agents, $P=R^{d}$ the price space, $C=R^{m}$ the commodity space, $f$ the price adjustment function, $\delta$ the price adjustment speed, $g$ the excess demand function, and the stochastic process $(p, X)$ the exchange process.

The phenomenon studied in this paper involves weakly interacting particles in the context of a dynamical system with fast and slow components. Probabilistic models with weakly interacting particles, where the behavior of a single particle is influenced by the large scale behavior of the whole set of particles, appear in [EK, Ku], and more explicitly in Oelschlager [O]. In our terms, the processes studied in [O] have the particles without the prices. There is a more extensive literature in probability theory on locally interacting particles, where the behavior of a single particle is influenced by its neighbors (see, for example, Liggett [L]). Dynamical systems with fast and slow components arise in the theory of 
singular perturbations (see, for example, [W, OM]). Nonstandard analysis has been exploited extensively in the area because of its power in handling different orders of infinity (see, e.g., [VDB]).

The existence and characterization of equilibria has long been a central object of study in mathematical economics. Economies with a large number of small traders are of particular interest. The main approaches to such economies are weak convergence of a sequence of large finite economies (Hildenbrand [Hi]), measure-theoretic methods where the economy has a continuum of traders $\mathrm{Au}$ mann $[\mathrm{Au}])$, or nonstandard economies with a hyperfinite set of traders and the Loeb measure (Brown and Robinson [BR]). The latter approach is particularly suited to our problem, because we need to consider relationships between several orders of infinity.

In this article we assume that a price equilibrium exists and study how one gets to the equilibrium. There is a variety of other approaches to this problem in the literature. Deterministic models for price adjustment given by differential or difference equations are surveyed in [Ha]. Another deterministic approach is in Smale [Sm]. Radner [Ra] surveys stochastic models for price adjustment. Gale [C] has introduced price adjustment models where the agents behave like locally interacting particles; there is no central warehouse, and the agents randomly meet and trade with their neighbors. The approach in this paper differs from the others in that the agents trade at random times, and the price and commodity vectors change together but with the price changing on a faster time scale.

For background in the use of nonstandard analysis in probability theory see [AFHL] or [SB]. The application of our result to mathematical economics was sketched in [K2] and will be presented in more detail in [K3].

Thanks are due to several colleagues for helpful discussions while this work was in progress, including Robert Anderson, Don Brown, Nigel Cutland, Ken Judd, Tom Kurtz, Jim Kuelbs, Lucinda Lewis, Peter Loeb, and Keith Stroyan. This research was supported in part by the National Science Foundation and the Vilas Trust Fund.

\section{AN INEQUALITY ON DIFFERENCE EQUATIONS}

In this section we shall obtain exponential bounds on the growth of solutions of difference equations which have fast and slow components and small persistent disturbances which are added at each time. In the remaining parts of the paper, we shall encounter equations of this kind where the small disturbances are random. In Theorem 1.8 we show that if the fast component is exponentially stable in the simplified equation where the slow component is held constant and the distrubances are suppressed, then in the full equation both components grow slowly.

Exponential bounds on the growth of difference (or differential) equations with small persistent disturbances are well known from the literature (see, e.g., [CL]). In the literature on perturbation theory (see, e.g., [W] or [OM]), one often studies the growth of solutions of differential equations with small parameters and/or fast and slow components. Equations with fast and slow components and small persistent disturbances are less common in the literature, but will be needed in this paper.

To prepare for our specific applications, we work with difference equations 
with values in a normed linear space rather than the more familiar context of differential equations with finitely many real variables. At the end of the section we reformulate the main result in a nonstandard setting which will be needed in $\S 5$.

Throughout this section we shall let $B$ and $C$ be normed linear spaces over the reals. The norm of an element $x$ of $B$ or $C$ is denoted by $\|x\|$. Let $\Delta t$ be a positive real number and let

$$
T=\{n \cdot \Delta t: n \in \mathbf{N} \text { and } 0<n\}
$$

be the set of all positive integer multiples of $\Delta t$. Thus the set $T$ depends on the parameter $\Delta t$. If $X: T \rightarrow B$, we denote the differences of $X$ by

$$
\Delta X(t)=X(t+\Delta t)-X(t) .
$$

We shall consider difference equations of the form

$$
X(0)=x_{0}, \quad \Delta X(t)=F(t, X(t)) \cdot \Delta t .
$$

To simplify notation, we shall first consider equations which have an equilibrium at zero, that is, $F(t, 0)=0$ for all $t$. At the end of this section we shall deal with the general case by making a change of variables.

Definition 1.1. Let $F$ be a function from $T \times B$ into $B . F$ is uniformly Lipschitz with parameter $c$ if for all $t, x$, and $y$,

$$
\|F(t, x)-F(t, y)\| \leq c \cdot\|x-y\| .
$$

The following lemma is a form of Gronwall's inequality.

Lemma 1.2. Let $F$ be a uniformly Lipschitz function with parameter a such that $F(t, 0)=0$ for all $t$. Let $J: T \rightarrow B$ be a function with $J(0)=0$, and let $X$ be the function from $T$ into $B$ given by the difference equation

$$
\Delta X(t)=F(t, X(t)) \cdot \Delta t+\Delta J(t) .
$$

Then for all $t \in T$,

$$
\|X(t)\| \leq e^{a t} \cdot[\|X(0)\|+\max \{\|J(s)\|: s \leq t\}]
$$

and

$$
\|X(t)-X(0)\| \leq e^{a t} \cdot \max \{\|J(s)\|: s \leq t\}+\left(e^{a t}-1\right) \cdot\|X(0)\| .
$$

Proof. Let $Z(0)=0$, and define $Z(t)$ by

$$
\Delta Z(t)=F(t, X(t)) \cdot \Delta t .
$$

Then $X(t)=Z(t)+X(0)+J(t)$. Let $H(t)=\|X(0)\|+\max \{\|J(s)\|: s \leq t\}$. Fix $t \in T$. We show by induction that for all $s \leq t$,

$$
\|Z(s)\| \leq\left(e^{a s}-1\right) \cdot H(t)
$$

and

$$
\|X(s)\| \leq e^{a s} \cdot H(t) .
$$


Both (1) and (2) are trivial for $s=0$. Assume that $s<t$ and that (1) and (2) hold for $s$. We first prove (1) for $s+\Delta t$, with the following string of inequalities:

$$
\begin{aligned}
\|Z(s+\Delta t)\| & =\|Z(s)+\Delta Z(s)\| \leq\|Z(s)\|+\|\Delta Z(s)\| \\
& \leq\left(e^{a s}-1\right) \cdot H(t)+a \cdot\|X(s)\| \cdot \Delta t \\
& \leq\left(e^{a s}-1\right) \cdot H(t)+a \cdot e^{a s} \cdot H(t) \cdot \Delta t \\
& =H(t) \cdot\left[e^{a s}(1+a \Delta t)-1\right] \leq H(t)\left[e^{a(s+\Delta t)}-1\right] .
\end{aligned}
$$

We now prove (2) for $s+\Delta t$. From the definition of $Z(s)$,

$$
X(s)=Z(s)+J(s)+X(0) .
$$

Then using (1) for $u=s+\Delta t$,

$$
\begin{aligned}
\|X(u)\| & =\|Z(u)+J(u)+X(0)\| \leq\|Z(u)\|+\|J(u)\|+\|X(0)\| \\
& \leq\left(e^{a u}-1\right) \cdot H(t)+H(t)=e^{a u} \cdot H(t) .
\end{aligned}
$$

This completes the induction and proves the first part of the lemma. The second part is proved from (1). We have

$$
\begin{aligned}
\|X(t)-X(0)\| & =\|Z(t)+J(t)\| \leq\|Z(t)\|+\|J(t)\| \\
& \leq\left(e^{a t}-1\right) \cdot H(t)+\max \{\|J(s)\|: s \leq t\} \\
& =\left(e^{a t}-1\right) \cdot\|X(0)\|+e^{a t} \cdot \max \{\|J(s)\|: s \leq t\} .
\end{aligned}
$$

This completes the proof.

We shall use the above lemma in the following form which is obtained by a change of variables.

Corollary 1.3. Suppose $F: T \times B \rightarrow B$ is uniformly Lipschitz with parameter $a$. Let $Z$ and $X$ be given by the difference equations

$$
\Delta Z(t)=F(t, Z(t)) \cdot \Delta t, \quad \Delta X(t)=F(t, X(t)) \cdot \Delta t+\Delta J(t) .
$$

If $t \leq u$ in $T$ and $\|J(s)-J(t)\| \leq \alpha$ for all $t \leq s \leq u$, then

$$
\|X(u)-Z(u)\| \leq e^{a(u-t)} \cdot(\|X(t)-Z(t)\|+\alpha)
$$

and

$$
\begin{aligned}
& \|(X(u)-Z(u))-(X(t)-Z(t))\| \\
& \quad \leq e^{a(u-t)} \cdot \alpha+\left(e^{a(u-t)}-1\right) \cdot\|X(t)-Z(t)\| .
\end{aligned}
$$

In what follows, we think of the function $J(t)$ as a small disturbance, and think of 0 as the undisturbed solution and $X(t)$ as a disturbed solution.

We now introduce the notion of a $\delta$-exponentially stable function $F$ and prove a stability lemma.

Definition 1.4. Let $\delta>0$. A function $F: T \times B \rightarrow B$ is said to be $\delta$ exponentially stable with parameters $b$ and $c$ (at zero) if $F(t, 0)=0$ for all $t$, and whenever $s \in T$ and $\|x\| \leq b$, we have

$$
\|X(s+c)\| \leq(\|x\|+\delta) / 4,
$$

where $X(u)$ for $u \geq s$ is given by the difference equation

$$
X(s)=x, \quad \Delta X(u)=F(u, X(u)) \cdot \Delta t .
$$


Proposition 1.5. Let $a, b$, and $c$ be positive real numbers. Then for every positive $\delta \leq b$, the following holds. Let $s \in T$ and let $X(t), s \leq t$, be given by the difference equation

$$
X(s)=x, \quad \Delta X(t)=F(t, X(t)) \cdot \Delta t+\Delta J(t)
$$

in a normed linear space $B$ where:

(a) $F$ is uniformly Lipschitz with parameter $a$;

(b) $F$ is $\delta$-exponentially stable with parameters $b$ and $c$; and

(c) $\|x\| \leq b$.

If $\|J(t)-J(u)\| \cdot e^{a c} \leq \delta / 4$ whenever $t, u \in[s, s+c]$, then

$$
\|X(s+c)\| \leq(\|x\|+\delta) / 2 .
$$

Proof. Let $Z(u)$ be given by the difference equation

$$
Z(s)=x, \quad \Delta Z(u)=F(u, Z(u)) \cdot \Delta t .
$$

By $\delta$-exponential stability $\|Z(s+c)\| \leq(\|x\|+\delta) / 4$. Let $\alpha=\delta /\left(4 e^{a c}\right)$. By Corollary 1.3 , we have

$$
\|X(s+c)-Z(s+c)\| \leq \alpha \cdot e^{a c} .
$$

Thus

$$
\begin{aligned}
\|X(s+c)\| & \leq(\|x\|+\delta) / 4+\alpha \cdot e^{2 a c} \\
& \leq(\|x\|+\delta) / 4+\delta / 4 \leq(\|x\|+\delta) / 2 .
\end{aligned}
$$

We shall now consider pairs of difference equations of the form

$$
\Delta X(t)=F(t, X(t), Y(t)) \cdot \Delta t, \quad \Delta Y(t)=G(t, X(t), Y(t)) \cdot \varepsilon \cdot \Delta t,
$$

where $\varepsilon$ is small. Intuitively, $X(t)$ will change infinitely fast compared to $Y(t)$. $X(t)$ takes values in the normed linear space $B$ and $Y(t)$ takes values in the normed linear space $C$. $F$ maps $T \times B \times C$ into $B$, and $G$ maps $T \times B \times C$ into $C$. By a change of variables we may transform the pair of equations into a pair of equations which has an equilibrium at zero, that is, $F(t, 0,0)=0$ and $G(t, 0,0)=0$ for all $t$. We shall concentrate on that case.

We first introduce a notion of exponential stability of a function $F(t, x, y)$ for small $y$.

Definition 1.6. We shall call a function $F: T \times B \times C \rightarrow B \quad \delta$-exponentially stable for small $y$ with parameters $b$ and $c$ if for all $s \in T, F(s, 0,0)=0$ and for all $x \in B$ and $y \in C$ with $\|x\| \leq b$ and $\|y\| \leq b$, we have

$$
\|W(s+c)-X(s+c)\| \leq(\|x\|+\delta) / 4,
$$

where $W(u)$ and $X(u)$ are given for $u \geq s$ by the difference equations

$$
\begin{aligned}
W(s) & =0, & \Delta W(u) & =F(u, W(u), y) \cdot \Delta t, \\
X(s) & =x, & \Delta X(u) & =F(u, X(u), y) \cdot \Delta t .
\end{aligned}
$$

For example, if $F(t, x, y)=H(t, x)$, where $H$ does not depend on $y$ and $H$ is $\delta$-exponentially stable, then $F$ is $\delta$-exponentially stable for small $y$ with the same parameters. If $F(t, x, y)$ is $\delta$-exponentially stable for small $y$ then the function $F(t, x, 0)$ is $\delta$-exponentially stable with the same parameters. We shall need the following modification of Proposition 1.5. 
Corollary 1.7. Let $a, b$, and $c$ be positive real numbers. Then for every positive $\delta \leq b$, the following holds. Let $s \in T$ and let $W(t)$ and $X(t)$ for $t \geq s$ be given by the difference equations

$$
\begin{gathered}
W(s)=0, \quad \Delta W(t)=F(t, W(t), y) \cdot \Delta t, \\
X(s)=x, \quad \Delta X(t)=F(t, X(t), y) \cdot \Delta t+\Delta J(t)
\end{gathered}
$$

is a normed linear space $B$ where:

(a) $F$ is uniformly Lipschitz with parameter $a$;

(b) $F$ is $\delta$-exponentially stable for small $y$ with parameters $b$ and $c$;

(c) $\|x\| \leq b$ and $\|y\| \leq b$; and

(d) $\|J(t)-J(s)\| \cdot e^{a c} \leq \delta / 4$ whenever $s \leq t \leq s+c$.

Then

$$
\|W(s+c)-X(s+c)\| \leq(\|x\|+\delta) / 2 .
$$

The proof is the same as the proof of Proposition 1.5.

Theorem 1.8. For all positive real numbers $a, b, c$, and $d$ there exist positive constants $A, \beta$, and $\lambda$ depending only on $a, b, c$, and $d$ such that the following holds. Let $0<\varepsilon \leq \delta \leq \lambda$. Let $\Delta t$ evenly divide $c$ and let $T$ be the set of multiples of $\Delta t$. Let $X$ and $Y$ be functions from $T$ into normed linear spaces $B$ and $C$ which satisfy the difference equations

$$
\begin{aligned}
& \Delta X(t)=F(t, X(t), Y(t)) \cdot \Delta t+\Delta J(t), \\
& \Delta Y(t)=\varepsilon \cdot[G(t, X(t), Y(t)) \cdot \Delta t+\Delta K(t)],
\end{aligned}
$$

where $F(t, 0,0)=0$ and $G(t, 0,0)=0$ for all $t$. Assume that

(a) $\|X(0)\| \leq \delta$ and $\|Y(0)\| \leq \delta$;

(b) $F$ and $G$ are uniformly Lipschitz in $(x, y)$ with parameters $a$ and $d$ respectively;

(c) $F$ is $\delta$-exponentially stable for small $y$ with parameters $b$ and $c$; and

(d) $\|J(t)-J(s)\| \leq \delta$ and $\|K(t)-K(s)\| \leq \delta$ whenever $|s-t| \leq c$.

Let $r$ be the solution of the equation $\delta \cdot A \cdot e^{\beta r \varepsilon}=b$. Then for all $t \leq r$,

(i) $\|X(t)\| \leq \delta \cdot A \cdot e^{\beta t \varepsilon}$ and

(ii) $\|Y(t)\| \leq \delta \cdot A \cdot e^{\beta t \varepsilon}$.

To prepare for the proof of Theorem 1.8 we prove a series of intermediate lemmas. For each of the lemmas, we assume that $0<\varepsilon \leq \delta$, that $X$ and $Y$ are solutions of the given difference equations, and that hypotheses (a)-(d) hold. The constants $A, \beta$, and $\lambda$ will not be used in the lemmas, and will be defined after the lemmas are proved. For convenience, we assume that $\delta \leq 1$. We shall consider an arbitrary element $t \in T$ which is held fixed, and consider values $u \in T$ such that $u \geq t$. We let $x=\|X(t)\|$ and $y=\|Y(t)\|$. Our first lemma gives a preliminary bound for $X(u)$.

Lemma 1.9. If $t \leq u \leq t+c$ in $T$, then

$$
\|X(u)\| \leq \exp [(a+\varepsilon \cdot d) \cdot c] \cdot[x+y+\delta+\varepsilon \cdot \delta] .
$$

This is proved by applying Corollary 1.3 to the pair $(X(t), Y(t))$.

We now obtain a bound for $Y(u)$. 
Lemma 1.10. Let $t \leq u \leq t+c$ in $T$ and suppose that $\|X(v)\| \leq M$ for all $t \leq v \leq u$. Then

$$
\|Y(u)\| \leq e^{\varepsilon d c} \cdot[y+\varepsilon \cdot(\delta+d \cdot M \cdot c)]
$$

and

$$
\|Y(u)-Y(t)\| \leq\left[e^{\varepsilon d c}-1\right] \cdot y+e^{\varepsilon d c} \cdot \varepsilon \cdot[\delta+d \cdot M \cdot c] .
$$

Proof. $Y$ satisfies the difference equation

$$
\Delta Y(u)=\varepsilon \cdot G(u, 0, Y(u)) \cdot \Delta t+\Delta I(u),
$$

where

$$
\Delta I(u)=\varepsilon \cdot \Delta K(u)+\varepsilon \cdot[G(u, X(u), Y(u))-G(u, 0, Y(u))] \cdot \Delta t .
$$

For each $u$,

$$
\|G(u, X(u), Y(u))-G(u, 0, Y(u))\| \leq d \cdot M .
$$

Therefore $\|I(u)-I(t)\| \leq \varepsilon \cdot(\delta+d \cdot M \cdot c)$. The result now follows from Corollary 1.3 .

Lemma 1.11. There is a constant $D$ depending only on $a, b, c$, and $d$ such that whenever $t \leq u \leq t+c$ in $T$,

$$
\|Y(u)-Y(t)\| \leq \varepsilon \cdot D \cdot[x+y+\delta] .
$$

Proof. Substitute the bound for $\|X(u)\|$ from Lemma 1.9 for $M$ in Lemma 1.10. We use hypothesis (d). To deal with the first term in the estimate for $\|Y(u)-Y(t)\|$ in Lemma 1.10, use the fact that since $\varepsilon \leq 1$,

$$
e^{c d \varepsilon}-1 \leq c \cdot d \cdot \varepsilon \cdot e^{c d \bar{\varepsilon}} \leq c \cdot d \cdot \varepsilon \cdot e^{c d} .
$$

We thus obtain

$$
D=e^{c d} \cdot[1+2 \cdot c \cdot d \cdot \exp (c \cdot(a+d))]
$$

Our next task is to obtain a better bound for $X(t+c)$. Define the function $Z(u), u \geq t$, by the difference equation

$$
Z(t)=0, \quad \Delta Z(u)=F(u, Z(u), Y(t)) \cdot \Delta t .
$$

Lemma 1.12. Let $E=4 \cdot a \cdot c \cdot e^{a c}$ be constant, and suppose $E \cdot y \leq b$. Then $\|Z(t+c)\| \leq E \cdot y / 2$.

Proof. The difference equation for $Z(u)$ may be written as

$$
\Delta Z(u)=F(u, Z(u), 0) \cdot \Delta t+\Delta H(u),
$$

where

$$
\Delta H(u)=[F(u, Z(u), Y(t))-F(u, Z(u), 0)] \cdot \Delta t .
$$

By the Lipschitz condition for $F, \Delta H(u) \leq a \cdot y \cdot \Delta t$, so $H$ has the property that $\|H(u)-H(s)\| \leq a \cdot y \cdot c$ whenever $|s-u| \leq c$. We have $a \cdot y \cdot c \cdot e^{a c}=E \cdot y / 4$. The result follows by Proposition 1.5 with $\delta=E \cdot y$.

Lemma 1.13. Let $D$ be the constant from Lemma 1.11. Let

$$
\sigma=4 \cdot e^{a c} \cdot[\delta+a \cdot c \cdot D \cdot \varepsilon \cdot(x+y+\delta)] .
$$


Suppose $x \leq b, \sigma \leq b$, and $y \leq b$. Then

$$
\|X(t+c)-Z(t+c)\| \leq(x+\sigma) / 2 .
$$

Proof. For $u \geq t, X(u)$ satisfies the difference equation

$$
\Delta X(u)=F(u, X(u), Y(t)) \cdot \Delta t+\Delta I(u),
$$

where $I(t)=0$ and

$$
\Delta I(u)=\Delta J(u)+[F(u, X(u), Y(u))-F(u, X(u), Y(t))] \cdot \Delta t .
$$

Thus in view of Lemma 1.11, for all $t \leq u \leq t+c$,

$$
\|I(u)-I(t)\| \leq \delta+a \cdot c \cdot\|Y(u)-Y(t)\| \leq \varphi,
$$

where $\varphi=\delta+a \cdot c \cdot D \cdot \varepsilon \cdot(x+y+\delta)$. Then $\sigma=4 \cdot e^{a c} \cdot \varphi$. The result now follows by Corollary 1.7 .

We now combine Lemmas 1.12 and 1.13 to get a bound for $\|X(t+c)\|$.

Lemma 1.14. Let $E$ be as in Lemma 1.12 and $\sigma$ be as in Lemma 1.13. Suppose $x, y, E \cdot y$, and $\sigma$ are all $\leq b$. Then

$$
\|X(t+c)\| \leq(x+E \cdot y+\sigma) / 2 .
$$

We are now ready to prove Theorem 1.8. We shall prove by induction on $t$ that, for appropriate constants $A_{1}, A_{2}, A_{3}, \beta$, and $\lambda$,

$$
\|X(t)\| \leq \delta \cdot A_{1} \cdot e^{\beta t \varepsilon}
$$

and

$$
\|Y(t)\| \leq \delta \cdot A_{2} \cdot e^{\beta t \varepsilon}
$$

whenever $\varepsilon \leq \delta \leq \lambda$ and $t \leq r$, where $\delta \cdot A_{3} \cdot e^{\beta r \varepsilon}=b$. During the course of the proof we shall keep track of the properties of $A_{1}, A_{2}, A_{3}, \beta$, and $\lambda$ which are needed. Our plan is to first prove (1) and (2) for all $t \leq c$, and then prove that if (1) and (2) hold for all $s<t+c$ then they hold for $t+c$. Since we have assumed that $\delta \leq 1$ in the lemmas, we require that $\lambda \leq 1$.

Suppose that $t \leq c$. By Lemma 1.9 with $(0, c)$ in place of $(t, s)$,

$$
\begin{aligned}
\|X(t)\| & \leq \exp [(a+\varepsilon \cdot d) \cdot c] \cdot[\|X(0)\|+\|Y(0)\|+\delta+\varepsilon \cdot \delta] \\
& \leq 4 \cdot \delta \cdot e^{(a+d) \cdot c} .
\end{aligned}
$$

We stipulate that

$$
A_{1} \geq 4 \cdot e^{(a+d) \cdot c} .
$$

Then (1) holds for $t \leq c$.

By Lemma 1.10 with $(0, t)$ in place of $(t, s)$,

$$
\begin{aligned}
\|Y(t)\| & \leq e^{t d \varepsilon} \cdot[\|Y(0)\|+\varepsilon \cdot(\delta+d \cdot M \cdot c)] \\
& \leq e^{t d \varepsilon} \cdot\left[\delta+\varepsilon \cdot\left(\delta+c \cdot d \cdot 4 \cdot \delta \cdot e^{(a+d) \cdot c}\right)\right] \\
& =\delta \cdot\left[1+\varepsilon \cdot\left(1+4 \cdot c \cdot d \cdot e^{(a+d)^{*} c}\right)\right] \cdot e^{t d \varepsilon} \\
& \leq \delta \cdot\left[2+4 \cdot c \cdot d \cdot e^{(a+d) \cdot c}\right] \cdot e^{t d \varepsilon} .
\end{aligned}
$$

We stipulate that

$$
A_{2} \geq 2+4 \cdot c \cdot d \cdot e^{(a+d) \cdot c}
$$


and

$$
\beta \geq d
$$

Then (2) holds for $t \leq c$.

Now assume that $u=t+c$ in $T$, that $t \leq r$, and that (1) and (2) hold for all $s<u$ in $T$. We shall prove that (1) and (2) hold for $u$. We adopt the notation for $x, y, E$, and $\sigma$ from the lemmas. Thus $x=\|X(t)\|$ and $y=\|Y(t)\|$.

In order to apply Lemma 1.14 , we must insure that $x, y, E \cdot y$, and $\sigma$ are $\leq b$. By assumption, $x \leq \delta \cdot A_{1} \cdot e^{\beta t \varepsilon}$ and $y \leq \delta \cdot A_{2} \cdot e^{\beta t \varepsilon}$. We stipulate that

$$
A_{1} \leq A_{3}, \quad A_{2} \leq A_{3} .
$$

Since $t \leq r, x \leq b$ and $y \leq b$. We recall that $E=4 \cdot a \cdot c \cdot e^{a c}$ and stipulate that

$$
E \cdot A_{2} \leq A_{3}
$$

so that $E \cdot y \leq b$. Recall that

$$
\sigma=4 \cdot e^{a c} \cdot[\delta+a \cdot c \cdot D \cdot \varepsilon \cdot(x+y+\delta)],
$$

where $D$ is a constant depending only on $a, b, c$, and $d$. Since $x, y \leq b$ and $\varepsilon \leq \delta$,

$$
\begin{aligned}
\sigma & \leq 4 \cdot e^{a c} \cdot[\delta+a \cdot c \cdot D \cdot \varepsilon \cdot(2 \cdot b+\delta)] \\
& \leq 4 \cdot e^{a c} \cdot \delta \cdot[1+a \cdot c \cdot D \cdot(2 \cdot b+\delta)] .
\end{aligned}
$$

We stipulate that $\lambda$ is small enough so that

$$
4 \cdot e^{a c} \cdot \lambda \cdot[1+a \cdot c \cdot D \cdot(2 \cdot b+\lambda)] \leq b .
$$

Then since $\delta \leq \lambda$, we have $\sigma \leq b$. By Lemma 1.14,

$$
\begin{aligned}
\|X(u)\| \leq & (x+E \cdot y+\sigma) / 2 \\
\leq & \delta \cdot A_{1} \cdot e^{\beta t \varepsilon} / 2+4 \cdot a \cdot c \cdot e^{a c} \cdot \delta \cdot A_{2} \cdot e^{\beta t \varepsilon} \\
& +4 \cdot e^{a c}[\delta+a \cdot c \cdot D \cdot \varepsilon \cdot(x+y+\delta)] \\
\leq & \delta \cdot e^{\beta t \varepsilon} \cdot\left[A_{1} / 2+4 \cdot A_{2} \cdot a \cdot c \cdot e^{a c}+4 \cdot \varepsilon \cdot a \cdot c \cdot D \cdot e^{a c} \cdot\left(A_{1}+A_{2}\right)\right] \\
& +\delta \cdot 4 \cdot\left(e^{a c}+a \cdot c \cdot D \cdot \varepsilon\right) \\
\leq & \delta \cdot e^{\beta t \varepsilon} \cdot\left[A_{1} / 2+4 \cdot A_{2} \cdot a \cdot c \cdot e^{a c}+e^{a c}\right. \\
& \left.\quad+4 \cdot \varepsilon \cdot a \cdot c \cdot D \cdot\left(e^{a c} \cdot\left(A_{1}+A_{2}\right)+1\right)\right] .
\end{aligned}
$$

We stipulate that

$$
A_{2} \cdot a \cdot c \cdot e^{a c}+e^{a c} \leq A_{1} / 4
$$

and

$$
4 \cdot a \cdot D \cdot\left(e^{a c} \cdot\left(A_{1}+A_{2}\right)+1\right) / A_{1} \leq \beta
$$

Then

$$
\|X(u)\| \leq \delta \cdot A_{1} \cdot e^{\beta t \varepsilon} \cdot(1+\varepsilon \cdot c \cdot \beta) \leq \delta \cdot A_{1} \cdot e^{\beta u \varepsilon} .
$$

Thus (1) holds for $u$.

We shall now apply Lemma 1.10. Since (1) holds for all $s \leq u$, we have

$$
\|X(s)\| \leq \delta \cdot A_{1} \cdot e^{\beta t \varepsilon} \cdot e^{\beta c \varepsilon}=M
$$


for all $s \leq u$. By Lemma 1.10

$$
\begin{aligned}
\|Y(u)\| & \leq e^{c d \varepsilon} \cdot[y+\varepsilon \cdot(\delta+M \cdot d \cdot c)] \\
& \leq e^{c d \varepsilon} \cdot\left[\delta \cdot A_{2} \cdot e^{\beta t \varepsilon}+\varepsilon \cdot\left(\delta+\delta \cdot A_{1} \cdot e^{\beta t \varepsilon} \cdot e^{\beta c \varepsilon} \cdot c \cdot d\right)\right] \\
& \leq \delta \cdot A_{2} \cdot e^{\beta t \varepsilon} \cdot e^{c d \varepsilon} \cdot\left[1+\varepsilon \cdot e^{\beta c \varepsilon} \cdot\left(1+\left(A_{1} / A_{2}\right) \cdot c \cdot d\right)\right] \\
& \leq \delta \cdot A_{2} \cdot e^{\beta t \varepsilon} \cdot e^{(c d+Q) \varepsilon},
\end{aligned}
$$

where $Q=e^{\beta c \varepsilon} \cdot\left(1+\left(A_{1} / A_{2}\right) \cdot c \cdot d\right)$. We must insure that $c \cdot d+Q \leq \beta \cdot c$. We thus want

$$
d+e^{\beta c \lambda} \cdot\left(1 / c+\left(A_{1} / A_{2}\right) \cdot d\right) \leq \beta .
$$

This can be accomplished by first setting a bound on $e^{\beta c \lambda}$, say $e^{\beta c \lambda} \leq 2$, then choosing $\beta$ sufficiently large, and then choosing $\lambda$ sufficiently small. We therefore stipulate that

$$
d+2 \cdot\left(1 / c+\left(A_{1} / A_{2}\right) \cdot d\right) \leq \beta
$$

and

$$
e^{\beta c \lambda} \leq 2 \text {. }
$$

It then follows that $\|Y(u)\| \leq \delta \cdot A_{2} \cdot e^{\beta t \varepsilon} \cdot e^{\beta c \varepsilon}$, so (2) holds for $u$.

By induction, if stipulations (3)-(12) hold then (1) and (2) hold for all $t \leq r$. By working in the right order, constants $A_{2}, A_{1}, A_{3}, \beta$, and $\lambda$ may be chosen depending only on $a, b, c$, and $d$ which satisfy (3)-(12). First choose $A_{2}$ large enough to satisfy (4). Then choose $A_{1}$ large enough to satisfy (3) and (9), then $A_{3}$ large enough to satisfy (6) and (7), then $\beta$ large enough to satisfy (5), (10), and (11), and finally choose $\lambda \leq 1$ small enough to satisfy (8) and (12). The proof is complete.

All of the results of this section still hold with no change in the proofs if time is cut off at some finite value $t_{1}$, that is, the sets $[0, \infty)$ and $T$ are everywhere replaced by $\left[0, t_{1}\right)$ and $T \cap\left[0, t_{1}\right)$.

We now reformulate Theorem 1.8 in a way which will be more convenient in $\S 4$. In Theorem 1.16, we modify the conditions on $F$ and $G$, and state the result in a nonstandard setting. The nonstandard setting will be needed in $\S 5$, and also allows us to simplify the statement of the result because it frees us from the need to give names for various bounds. Up to this point we have considered small perturbations of difference equations which have a zero solution, because of the hypothesis that $F(t, 0,0)=G(t, 0,0)=0$. Now we drop this hypothesis and consider the case of a small perturbation of an arbitrary solution. To keep things simple, we restrict Theorem 1.16 to the autonomous case where $F$ and $G$ do not depend on $t$, but the proof will use Theorem 1.8 in the nonautonomous case. The direct analogue of the notion of exponential stability near zero is a rather complicated notion of exponential stability near a path which depends on $t$. Instead, we shall introduce a simpler notion of exponential stability near a point. The cost of this simpler notion of exponential stability will be an additional hypothesis in Theorem 1.16 that the function $G$ is linearly bounded.

Definition 1.15. We shall call a function $F: B \times C \rightarrow B$ exponentially stable near a point $\left(x_{0}, y_{0}\right) \in B \times C$ with parameters $b$ and $c$ if, for all $x$ and $y$ 
with $\left\|x-x_{0}\right\| \leq b$ and $\left\|y-y_{0}\right\| \leq b$, we have

$$
\|W(c)-X(c)\| \leq\left\|x-x_{0}\right\| / 4,
$$

where $W(u)$ and $X(u)$ are given by the difference equations

$$
\begin{array}{cl}
W(0)=x_{0}, & \Delta W(u)=F(W(u), y) \cdot \Delta t, \\
X(0)=x, & \Delta X(u)=F(X(u), y) \cdot \Delta t .
\end{array}
$$

If $D \subseteq C$ and the above holds for all $y \in D$, we call $F$ exponentially stable for $D$ near $\left(x_{0}, y_{0}\right)$.

Theorem 1.16. Suppose $\varepsilon, \Delta t$, and $\Delta t / \varepsilon$ are positive infinitesimal, and let $T$ be an initial segment of the set of positive hyperinteger multiples $t$ of $\Delta t$. Let $X, X^{\prime}$ be functions from $T$ into ${ }^{*} B$, and $Y, Y^{\prime}$ be functions from $T$ into ${ }^{*} C$, which satisfy the difference equations

$$
\begin{aligned}
& \Delta X(t)=F(X(t), Y(t)) \cdot \Delta t+\Delta J(t), \\
& \Delta Y(t)=\varepsilon \cdot[G(X(t), Y(t)) \cdot \Delta t+\Delta K(t)], \\
& \Delta X^{\prime}(t)=F\left(X^{\prime}(t), Y^{\prime}(t)\right) \cdot \Delta t \\
& \Delta Y^{\prime}(t)=\varepsilon \cdot G\left(X^{\prime}(t), Y^{\prime}(t)\right) \cdot \Delta t
\end{aligned}
$$

Assume that

(a) $\left\|X(0)-X^{\prime}(0)\right\| \approx 0$ and $\left\|Y(0)-Y^{\prime}(0)\right\| \approx 0$;

(b) $F:{ }^{*} B \times{ }^{*} C \rightarrow{ }^{*} B$ and $G:{ }^{*} B \times{ }^{*} C \rightarrow{ }^{*} C$ are internal and uniformly Lipschitz with finite Lipschitz bound;

(c) for each $t \in T, F$ is *-exponentially stable near the point $\left(X^{\prime}(t), Y^{\prime}(t)\right)$ with positive real parameters $b$ and $c$;

(d) $\|J(t)-J(s)\| \approx 0$ and $\|K(t)-K(s)\| \approx 0$ whenever $|s-t|$ is finite; and

(e) $\left\|Y^{\prime}(0)\right\|$ is finite, and there is a finite $d$ such that $\|G(x, y)\| \leq d \cdot(\|y\|+$ 1) for all $y \in{ }^{*} C$.

Then for all $t \in T$ such that $t \cdot \varepsilon$ is finite,

$$
\left\|X(t)-X^{\prime}(t)\right\| \approx 0 \text { and }\left\|Y(t)-Y^{\prime}(t)\right\| \approx 0 .
$$

Proof. We may assume without loss of generality that the set $\{t \cdot \varepsilon: t \in T\}$ has a finite bound $k$. We shall apply the transfer of Theorem 1.8. Make the following changes of variables:

$$
\begin{gathered}
\bar{X}(t)=X(t)-X^{\prime}(t), \quad \bar{Y}(t)=Y(t)-Y^{\prime}(t), \\
\bar{F}(t, x, y)=F\left(x+X^{\prime}(t), y+Y^{\prime}(t)\right)-F\left(X^{\prime}(t), Y^{\prime}(t)\right), \\
\bar{G}(t, x, y)=G\left(x+X^{\prime}(t), y+Y^{\prime}(t)\right)-G\left(X^{\prime}(t), Y^{\prime}(t)\right) .
\end{gathered}
$$

Then $\bar{F}(t, 0,0)=0, \bar{G}(t, 0,0)=0$, and

$$
\begin{gathered}
\Delta \bar{X}(t)=\bar{F}(t, \bar{X}(t), \bar{Y}(t)) \Delta t+\Delta J(t), \\
\Delta \bar{Y}(t)=\varepsilon \cdot[\bar{G}(t, \bar{X}(t), \bar{Y}(t)) \Delta t+\Delta K(t)] .
\end{gathered}
$$

With these new variables, hypotheses (a), (b), and (d) of Theorem 1.8 hold for any sufficiently large infinitesimal $\delta$. Using the extra hypothesis (e), we shall show that hypothesis (c) of Theorem 1.8 holds for sufficiently large infinitesimal $\delta$. 
Let $s \in T$ and $\|x\|,\|y\| \leq b$. We must show that

$$
\|\bar{W}(s+c)-\bar{Z}(s+c)\| \leq(\|x\|+\delta) / 4,
$$

where

$$
\begin{aligned}
\bar{W}(s) & =0, & \Delta \bar{W}(u) & =\bar{F}(u, \bar{W}(u), y) \cdot \Delta t, \\
\bar{Z}(s) & =x, & \Delta \bar{Z}(u) & =\bar{F}(u, \bar{Z}(u), y) \cdot \Delta t .
\end{aligned}
$$

Let

$$
\begin{array}{cr}
W(s)=X^{\prime}(s), \quad \Delta W(u)=F\left(W(u), y+Y^{\prime}(s)\right) \cdot \Delta t, \\
Z(s)=x+X^{\prime}(s), \quad \Delta Z(u)=F\left(Z(u), y+Y^{\prime}(s)\right) \cdot \Delta t .
\end{array}
$$

By hypothesis (c) of this theorem,

$$
\|W(s+c)-Z(s+c)\| \leq\|x\| / 4 .
$$

Now let $W^{\prime}(u)=\bar{W}(u)+X^{\prime}(u)$ and $Z^{\prime}(u)=\bar{Z}(u)+X^{\prime}(u)$. Then

$$
\bar{W}(s+c)-\bar{Z}(s+c)=W^{\prime}(s+c)-Z^{\prime}(s+c) .
$$

Unraveling the definitions, we see that $W^{\prime}$ and $Z^{\prime}$ satisfy the difference equations

$$
\begin{array}{cr}
W^{\prime}(s)=X^{\prime}(s), & \Delta W(u)=F\left(W^{\prime}(u), y+Y^{\prime}(u)\right) \cdot \Delta t \\
Z^{\prime}(s)=x+X^{\prime}(s), & \Delta Z(u)=F\left(Z^{\prime}(u), y+Y^{\prime}(u)\right) \cdot \Delta t
\end{array}
$$

Let

$$
\begin{aligned}
\Delta H(u) & =\left[F\left(W^{\prime}(u), y+Y^{\prime}(u)\right)-F\left(W^{\prime}(u), y+Y^{\prime}(s)\right)\right] \cdot \Delta t \\
\Delta I(u) & =\left[F\left(Z^{\prime}(u), y+Y^{\prime}(u)\right)-F\left(Z^{\prime}(u), y+Y^{\prime}(s)\right)\right] \cdot \Delta t
\end{aligned}
$$

Then

$$
\begin{gathered}
\Delta W^{\prime}(u)=F\left(W^{\prime}(u), y+Y^{\prime}(s)\right) \cdot \Delta t+\Delta H(u), \\
\Delta Z^{\prime}(u)=F\left(Z^{\prime}(u), y+Y^{\prime}(s)\right) \cdot \Delta t+\Delta I(u) .
\end{gathered}
$$

We shall use Corollary 1.3 to get bounds on $\left\|W(s+c)-W^{\prime}(s+c)\right\|$ and $\left\|Z(s+c)-Z^{\prime}(s+c)\right\|$. Using (e), it follows by induction on $u$ that

$$
\left\|Y^{\prime}(u)\right\| \leq\left\|Y^{\prime}(0)\right\| \cdot e^{2 \varepsilon u d} \leq\left\|Y^{\prime}(0)\right\| \cdot e^{2 k d} .
$$

Then using (e) again, whenever $u \in[s, s+c]$,

$$
\left\|Y^{\prime}(u)-Y^{\prime}(s)\right\| \leq 2 \varepsilon c d \cdot\left\|Y^{\prime}(0)\right\| \cdot e^{2 k d} .
$$

Thus if $a$ is the Lipschitz bound for $F$, for all $u, v \in[s, s+c]$ we have

$$
\|H(u)-H(v)\| \leq j \cdot \varepsilon, \quad\|I(u)-I(v)\| \leq j \cdot \varepsilon,
$$

where $j=2 a c^{2} d \cdot\left\|Y^{\prime}(0)\right\| \cdot e^{2 k d}$ is finite. By Corollary 1.3,

$$
\left\|W(s+c)-W^{\prime}(s+c)\right\| \leq e^{a c} \cdot j \cdot \varepsilon,
$$

and similarly for $Z$. Therefore for any infinitesimal $\delta$ such that $\delta \geq 8 e^{a c} \cdot j \cdot \varepsilon$, we have

$$
\begin{aligned}
& \|\bar{W}(s+c)-\bar{Z}(s+c)\| \leq\|W(s+c)-Z(s+c)\| \\
& \quad+\left\|W(s+c)-W^{\prime}(s+c)\right\|+\left\|Z(s+c)-Z^{\prime}(s+c)\right\| \\
& \quad \leq(\|x\|+\delta) / 4
\end{aligned}
$$


This proves hypothesis (c) of Theorem 1.8. The desired conclusion now follows from Theorem 1.8.

The following corollary is obtained from Theorem 1.16 by a change in the time scale, replacing $t$ by $t \cdot \varepsilon$ and $K$ by $K \cdot \varepsilon$.

Theorem 1.17. Suppose $\varepsilon, \Delta t$, and $\Delta t / \varepsilon$ are positive infinitesimal, and let $T$ be an initial segment of the set of multiples $t$ of $\Delta t$. Let $X, X^{\prime}$ be functions from $T$ into ${ }^{*} B$, and $Y, Y^{\prime}$ be functions from $T$ into ${ }^{*} C$, which satisfy the difference equations

$$
\begin{aligned}
& \Delta X(t)=F(X(t), Y(t)) \cdot \Delta t / \varepsilon+\Delta J(t), \\
& \Delta Y(t)=G(X(t), Y(t)) \cdot \Delta t+\Delta K(t), \\
& \Delta X^{\prime}(t)=F\left(X^{\prime}(t), Y^{\prime}(t)\right) \cdot \Delta t / \varepsilon \\
& \Delta Y^{\prime}(t)=G\left(X^{\prime}(t), Y^{\prime}(t)\right) \cdot \Delta t .
\end{aligned}
$$

Assume that

(a) $\left\|X(0)-X^{\prime}(0)\right\| \approx 0$ and $\left\|Y(0)-Y^{\prime}(0)\right\| \approx 0$;

(b) $F:{ }^{*} B \times{ }^{*} C \rightarrow{ }^{*} B$ and $G:{ }^{*} B \times{ }^{*} C \rightarrow{ }^{*} C$ are internal and uniformly Lipschitz with finite Lipschitz bound;

(c) for each $t \in T, F$ is *-exponentially stable near the point $\left(X^{\prime}(t), Y^{\prime}(t)\right)$ with positive real parameters;

(d) $\|J(t)-J(u)\| \approx 0$ and $\|K(t)-K(u)\| / \varepsilon \approx 0$ whenever $t, u \in T$ and $|t-u| \leq \varepsilon ;$ and

(e) $\left\|Y^{\prime}(0)\right\|$ is finite, and there is a finite $d$ such that $\|G(x, y)\| \leq d(\|y\|+1)$ for all $y \in{ }^{*} C$.

Then for all finite $t \in T$,

$$
\left\|X(t)-X^{\prime}(t)\right\| \approx 0 \text { and }\left\|Y(t)-Y^{\prime}(t)\right\| \approx 0 .
$$

Before proceeding to the next section we shall state one more inequality which will be needed later. This result, Bernstein's inequality, is probabilistic in nature and is not related to the rest of this section. The statement below and a proof are in [K1].

Bernstein's inequality 1.18. Let $\Omega$ be a probability space, let $\mathbf{F}_{t}, t \in T$, be an increasing family of $\sigma$-algebras on $\Omega$, let $\alpha, K$, and $\sigma$ be positive real numbers with $\alpha<1$, and let $M(\omega, t)$ be a martingale on $\Omega$ with respect to $\mathbf{F}_{t}$ such that

(i) $M(\omega, 0)=0$ for all $\omega$;

(ii) $|\Delta M(\omega, t)| \leq K$ for all $\omega$ and $t$; and

(iii) $E\left[(\Delta M(\cdot, t))^{2} \mid \mathbf{F}_{t}\right](\omega) \leq \sigma^{2} \cdot \Delta t$ for all $\omega$ and $t$.

Then whenever $t \in T$ and

$$
0<z \leq \sigma \cdot \log (2-\alpha) \cdot \sqrt{t} / K
$$

we have

$$
P\left[\max _{s<t}|M(\omega, s)| \geq z \cdot \sigma \cdot \sqrt{t}\right] \leq 2 \cdot e^{-\alpha \cdot z^{2} / 2} .
$$

In the above inequality, $\Delta M$ denotes the change in $M$ from times $t$ to $t+\Delta t$

$$
\Delta M(\omega, t)=M(\omega, t+\Delta t)-M(\omega, t)
$$




\section{Finite EXChANGE PROCESSES}

Let $d$ and $m$ be positive integers, which will remain fixed throughout this paper. We shall call the Euclidean space $P=R^{d}$ the price space, and the Euclidean space $C=R^{m}$ the commodity space. For $x \in P$ or $R^{m}$, let $|x|$ be the supremum of the absolute values of the coordinates of $x$.

We shall now introduce a discrete time Markov process which we call a finite exchange process.

Let $A$ and $\Gamma$ be finite sets. We call $A$ the set of agents and $\Gamma$ the set of choices. To simplify notation, we shall write $A$ instead of $\#(A)$ for the number of elements of the set $A$. Let $A^{\prime}=A \cup\{0\}$, where $\circ$ is not an element of $A$. Let $0<\Delta t \leq 1 / A$ and let $T=\{K \cdot \Delta t: K \in N\}$ be a discrete time line. Give $A^{\prime}$ the measure where $\circ$ has measure $1-A \cdot \Delta t$ and each $a \in A$ has measure $\Delta t$. Consider the sample space $\Omega=\left(A^{\prime} \times \Gamma\right)^{T}$ with the product measure. Each $\omega \in \Omega$ has the form $\omega=\left(\omega_{\Gamma}, \omega_{A}\right)$, where $\omega_{\Gamma} \in \Gamma^{T}$ and $\omega_{A} \in A^{\prime T} . \omega_{A}(t+\Delta t)=a$ means that the agent $a$ is chosen to move at time $t$, and $\omega_{\Gamma}(t+\Delta t)$ determines where $a$ moves.

Let $f$ and $g$ be functions

$$
f: \Gamma \times P \times C \rightarrow P, \quad g: \Gamma \times P \times C \rightarrow C .
$$

We call $f$ the price adjustment function and $g$ the excess demand function.

Let $\delta$ be a positive real number which remains fixed. We call $\delta$ the price adjustment parameter.

We define two stochastic processes $p: \Omega \times T \rightarrow P$ and $X: \Omega \times A \times T \rightarrow C$. We call $p(\omega, t)$ the price vector at time $t$, and $X(\omega, a, t)$ the commodity vector of agent $a$ at time $t$. The initial values are $p_{0}=p(\omega, 0)$ and $X_{0}(a)=$ $X(\omega, a, 0)$, where $p_{0} \in P$ and $X_{0}(a) \in C$ for each $a \in A$. The changes in $p$ and $X$ from time $t$ to $t+\Delta t$ are

$$
\begin{gathered}
\Delta p(\omega, t)= \begin{cases}0 & \text { if } \omega_{A}(t+\Delta t)=\circ, \\
\delta \cdot f\left(\omega_{\Gamma}(t+\Delta t), p(\omega, t), X(\cdot, a, t)\right) & \text { if } \omega_{A}(t+\Delta t)=a,\end{cases} \\
\Delta X(\omega, a, t)= \begin{cases}0 & \text { if } \omega_{A}(t+\Delta t) \neq a, \\
g\left(\omega_{\Gamma}(t+\Delta t), p(\omega, t), X(\omega, a, t)\right) & \text { if } \omega_{A}(t+\Delta t)=a .\end{cases}
\end{gathered}
$$

Let $\mathbf{F}_{t}$ denote the algebra of subsets of $\Omega$ determined by the equivalence relation $\omega\left|t=\omega^{\prime}\right| t$. For each $t$, this equivalence relation has only finitely many equivalence classes. For each $a$ and $t, p(\cdot, t)$ and $X(\cdot, a, t)$ are $\mathbf{F}_{t^{-}}$ measurable. Moreover, the pair $(p, X)$ is a Markov process with respect to $\mathbf{F}_{t}$.

The function $f$ is uniformly Lipschitz on $P \times C$ with bound $k$ if for all $x, y \in C, p, q \in P$, and $\gamma \in \Gamma$,

$$
|f(\gamma, p, x)-f(\gamma, q, y)| \leq k \cdot(|x-y|+|p-q|) .
$$

$k$ is a linear bound of $f$ on $C$ if for all $x \in C, p \in P$, and $\gamma \in \Gamma$,

$$
|f(\gamma, p, x)| \leq k \cdot(1+|x|) .
$$

Definition. The stochastic process $(p, X)$ described in the preceding paragraphs is called a finite exchange process with Lipschitz bound $k$ if the functions $f$ and $g$ are uniformly Lipschitz on $P \times C$ with bound $k$ and linearly bounded by $k$ on $C$. 
A finite exchange process $(p, X)$ is determined by the sets $A$ and $\Gamma$, the numbers $\Delta t, d, m$, and $\delta$, the functions $f$ and $g$, and the initial values $p_{0}$ and $X_{0}(\cdot)$.

For each function $Y: A \rightarrow C$, let $b_{Y}$ be the probability measure over $C$ induced by $Y$ and the counting measure over $A$, that is, each point $x \in C$ has weight $\#\left(Y^{-1}\{x\}\right) / A$.

The aim of this paper is to prove a law of large numbers of the following type for exchange processes. Given $l \in(0,1)$ and $\varepsilon, t_{1}>0$, if $A$ is sufficiently large and $\delta=A^{-l}$, then with probability at least $1-\varepsilon$ the price $p(\omega, t)$ and commodity distribution $b_{X(\omega, \bullet, t)}$ are within $\varepsilon$ of their expected values for all times $t<t_{1}$.

In order to formulate the result precisely, we shall introduce the state space and represent the commodity distribution $b_{X(\omega, \cdot, t)}$ as an element of the state space. $\S \S 3$ and 4 contain technical lemmas which will be needed for our main theorem, which will be proved in $\S 5$. In $\S \S 4$ and 5 we work in a nonstandard setting so that we can keep track of the relation between several small quantities involved in the theorem.

The state space $\mathbf{B}$ will be a particular Banach space that depends on the commodity dimension $m$. Recall that $C$ is the Euclidean space $C=R^{m}$.

Let $\mathbf{L}$ be the linear space of all functions $\varphi: C \rightarrow R$ which are uniformly Lipschitz and linearly bounded on $C$. Denote the Lipschitz bound by

$$
k_{\varphi}=\sup \{|\varphi(x)-\varphi(y)| /|x-y|: x \neq y \text { in } C\},
$$

and the linear bound by

$$
\||\varphi|\|=\sup \{|\varphi(x)| /(1+|x|): x \in C\} .
$$

Let $\|\varphi\|$ be the maximum of $k_{\varphi}$ and $\|\varphi\| \|$.

The state space $\mathbf{B}$ is defined as the dual space of the linear space $(\mathbf{L},\|\|)$. Then for $b \in \mathbf{B}$,

$$
\|b\|=\sup \{|b(\varphi)|: \varphi \in \mathbf{L},\|\varphi\| \leq 1\}
$$

is finite, and $(\mathbf{B},\|\|)$ is a Banach space. For $b \in \mathbf{B}$, let

$$
\|b \mid\|=\sup \{|b(\varphi)|:|\|\varphi \mid\| \leq 1\} .
$$

Thus $\|b\| \leq\||| b\| \mid \leq \infty$.

Let $\mathbf{M}$ be the set of all Borel probability measures $b$ on $C$ such that $\int|x| d b<\infty$, and for each $b \in \mathbf{M}$ and $\varphi \in \mathbf{L}$ let $b(\varphi)=\int \varphi d b$. Then each $b \in \mathbf{M}$ is a linear functional on $\mathbf{L}$. Each $b \in \mathbf{M}$ is also bounded, because if $\|\varphi\| \leq k$ then $|\varphi| \leq k \cdot(1+|x|)$ and therefore

$$
|b(\varphi)| \leq k \int 1+|x| d b .
$$

Thus $\mathbf{M}$ is a subset of the state space $\mathbf{B}$.

Whenever we refer to topological concepts or Lipschitz conditions involving the spaces $\mathbf{M}$ or $\mathbf{B}$, they will be understood to be in the sense of the \|\| -norm.

Let $E_{A}$ and $E_{\Gamma}$ denote the expected values with respect to the sets $A$ and $\Gamma$ with the counting measure. Let $E_{\Omega}$ be the expected value with respect to the set $\Omega$ with the previously described product measure. We shall use the notation $E_{\Omega}\left[\cdots \mid \mathbf{F}_{t}\right](\omega)$ for conditional expectation on $\Omega$ with respect to $\mathbf{F}_{t}$. If $f$ is an 
$\mathbf{F}_{t}$-measurable function from $\Omega$ into $\mathbf{B}$, the expected value $E_{\Omega}[f(\cdot)]$ is just a finite sum of elements of $\mathbf{B}$,

$$
E_{\Omega}[f(\cdot)]=\sum\left[f(\gamma) \cdot P(\gamma): \gamma \text { is an equivalence class of } \mathbf{F}_{t}\right] .
$$

Any function $Y: A \rightarrow C$ determines a measure $b_{Y} \in \mathbf{M}$ defined by $b_{Y}(\varphi)=$ $\sum_{a \in A} \varphi(Y(a)) / A=E_{A}[\varphi(Y(\cdot))]$.

Given a finite exchange process $(p, X)$, we define the process $B: \Omega \times T \rightarrow$ $\mathbf{M}$ by $B(\omega, t)=b_{X(\omega, \cdot, t)}$ and call $B(\omega, t)$ the commodity distribution, or commodity state, at time $t$. The value of $B(\omega, 0)$ does not depend on $\omega$ and is denoted by $B_{0}$. Thus $B(\omega, 0)=B_{0}=b_{X_{0}(\cdot)}$.

Our aim in this section is to prove the following theorem.

Theorem 2.1. In a finite exchange process $(p, X)$, the price vector $p(\omega, t)$ and commodity state $B(\omega, t)$ satisfy a pair of stochastic difference equations

$$
\begin{gathered}
E_{\Omega}\left[\Delta p(\cdot, t) \mid \mathbf{F}_{t}\right](\omega)=F(p(\omega, t), B(\omega, t)) \cdot \Delta t / \varepsilon, \\
E_{\Omega}\left[\Delta B(\cdot, t) \mid \mathbf{F}_{t}\right](\omega)=G(p(\omega, t), B(\omega, t)) \cdot \Delta t,
\end{gathered}
$$

where $\varepsilon=1 /(A \cdot \delta)$ and $F: P \times \mathbf{M} \rightarrow P$ and $G: P \times \mathbf{M} \rightarrow \mathbf{B}$ are functions which are Lipschitz on $P$ and $\mathbf{M}$ with respect to the \|\|$-$ metric and depend only on the functions $f$ and $g$ (not on $A, \delta, \Delta t$, or the initial values).

Notice that if the price adjustment parameter $\delta$ is small, then $\Delta t / \varepsilon$ is small because $\Delta t / \varepsilon=A \cdot \Delta t \cdot \delta \leq \delta$. Moreover, if $\varepsilon$ is also small then $\Delta t / \varepsilon$ is large compared to $\Delta t$.

Before beginning the proof, we digress to give a preview of how we plan to use Theorem 2.1. The processes $p(\omega, t)$ and $B(\omega, t)$ may be written in the form

$$
\begin{array}{cc}
p(\omega, 0)=p_{0}, & \Delta p(\omega, t)=F(p(\omega, t), B(\omega, t)) \cdot \Delta t / \varepsilon+\Delta M(\omega, t), \\
B(\omega, 0)=B_{0}, & \Delta B(\omega, t)=G(p(\omega, t), B(\omega, t)) \cdot \Delta t+\Delta N(\omega, t),
\end{array}
$$

where $M$ and $N$ are martingales with $M(\omega, 0)=0$ and $N(\omega, 0)=0$. We then consider the corresponding deterministic difference equations obtained by leaving out the terms $\Delta M$ and $\Delta N$,

$$
\begin{gathered}
p_{1}(0)=p_{0}, \quad \Delta p_{1}(t)=F\left(p_{1}(t), B_{1}(t)\right) \cdot \Delta t / \varepsilon, \\
B_{1}(0)=B_{0}, \quad \Delta B_{1}(t)=G\left(p_{1}(t), B_{1}(t)\right) \cdot \Delta t .
\end{gathered}
$$

Beginning in $\S 4$, we shall pass from a finite exchange process to a starfinite exchange process, that is, the transfer of the notion of a finite exchange process in the sense of nonstandard analysis. We shall take the set $A$ to be hyperfinite and take $\delta$ and $\varepsilon$ to be infinitesimal. Thus the process $p(\omega, t)$ will move at a rate which is infinitely fast compared to $B(\omega, t)$. Our aim will be to apply Theorem 1.17 to show that for some positive real time $t_{1}$ we have

$$
p(\omega, t) \approx p_{1}(t), \quad B(\omega, t) \approx B_{1}(t) \text { for all } t<t_{1}
$$

with Loeb probability one. Hypothesis (a) of 1.17 holds because $(p, B)$ and $\left(p_{1}, B_{1}\right)$ have the same starting point. In the present section we shall prove hypotheses (b) and (e) of 1.17, that the functions $F$ and $G$ are Lipschitz and $G$ is linearly bounded. In $\S 4$ we shall use Bernstein's Inequality to prove 
hypothesis (d) of 1.17 , that $M(t) \approx M(u)$ and $N(t) / \varepsilon \approx N(u) / \varepsilon$ whenever $t$ is within $\varepsilon$ of $u$. Then only the exponential stability hypothesis (c) of 1.17 is needed to get the desired conclusion. We tie things together in $\S 5$. The main result, Theorem 5.4 , is that if the function $F$ is exponentially stable near $\left(p_{1}(t), B_{1}(t)\right)$ with real parameters whenever $\mathrm{st}(t)<t_{1}$, then

$$
p(\omega, t) \approx p_{1}(t), \quad B(\omega, t) \approx B_{1}(t) \quad \text { whenever st }(t)<t_{1}
$$

with Loeb probability one. It will follow (Theorem 5.6) that with Loeb probability one, $p(\omega, t)$ and $B(\omega, t)$ are infinitely close to their expected values whenever $\operatorname{st}(t)<t_{1}$. This is a law of large numbers for the processes $p$ and $B$. It shows that for each real $\alpha>0$ there exists an integer $n$ such that for any finite $A$ with more than $n$ elements, the finite exchange process has the property that with probability at least $1-\alpha, p(\omega, t)$ and $B(\omega, t)$ are within $\alpha$ of their expected values for all $t<t_{1}-\alpha$.

This concludes our preview. We now begin a series of lemmas leading to the proof of Theorem 2.1.

Assumption. We assume throughout this section that $f: \Gamma \times P \times C \rightarrow P$ and $g: \Gamma \times P \times C \rightarrow C$ are uniformly Lipschitz on $P \times C$ with bound $k$ and linearly bounded on $C$ with bound $K$.

Definition. Let $\mathbf{B}_{+}$be the set of all nonnegative definite elements of $\mathbf{B}$, that is,

$$
\mathbf{B}_{+}=\{b \in \mathbf{B}: b(\varphi) \geq 0 \text { whenever } \varphi \geq 0\} \text {. }
$$

The following lemma is easily checked.

Lemma 2.2. (i) $\mathbf{M}$ is a convex subset of $\mathbf{B}_{+}$.

(ii) $\mathbf{B}_{+}$is convex and closed in $(\mathbf{B},\|\|)$.

It will be convenient to define the functions $F$ and $G$ on $P \times \mathbf{B}$ instead of on $P \times \mathbf{M}$, and prove that they are Lipschitz on $P \times \mathbf{B}_{+}$.

Let abs be the particular function $\operatorname{abs}(x)=1+|x|$. Thus abs $\in \mathbf{L}$, and $\|\mathrm{abs}\|=\|$ |abs $\|=1$.

Lemma 2.3. For all $b \in \mathbf{B}_{+},\|b\|=\|\mid b\| \|=b$ (abs).

Proof. We have $0 \leq b($ abs $) \leq\|b\| \leq\|b\| \|$. Let $0 \leq n<\|\mid b\| \| \leq \infty$. Then for some $\varphi \in \mathbf{L}$ with $\|\varphi\||\leq 1, n<| b(\varphi) \mid$. Let $\varphi^{+}=\max (\varphi, 0)$ and $\varphi^{-}=\min (\varphi, 0)$, so that $\varphi=\varphi^{+}+\varphi^{-}$and $\varphi^{-} \leq 0 \leq \varphi^{+}$. Then $\left\|\varphi^{-}\right\| \leq \leq 1$ and $\left\|\varphi^{+}\right\| \mid \leq 1$. Therefore

$$
b\left(\varphi^{-}\right) \leq 0 \leq b\left(\varphi^{+}\right) \text {and } b(\varphi)=b\left(\varphi^{-}\right)+b\left(\varphi^{+}\right) .
$$

It follows that

$$
|b(\varphi)| \leq \max \left(\left|b\left(\varphi^{-}\right)\right|,\left|b\left(\varphi^{+}\right)\right|\right) .
$$

Suppose $|b(\varphi)| \leq\left|b\left(\varphi^{+}\right)\right|$. Then $n<b\left(\varphi^{+}\right)$. Since $\left\|\left|\varphi^{+}\right|\right\| \leq 1$, we have abs $-\varphi^{+} \geq 0$, and hence

$$
0 \leq b\left(\mathrm{abs}-\varphi^{+}\right)=b(\mathrm{abs})-b\left(\varphi^{+}\right),
$$

so $b\left(\varphi^{+}\right) \leq b($ abs $)$ and $n<b($ abs $)$. Since this holds for all $n<\||| b\| \|$, we have $|\| b||| \leq b($ abs $)$. The case $|b(\varphi)| \leq\left|b\left(\varphi^{-}\right)\right|$is similar.

The next definition introduces an auxiliary function $\bar{f}$ which we shall use to define the Lipschitz function $F: P \times \mathbf{B}_{+} \rightarrow P$. 
Definition. For each $p \in P$, define $\bar{f}(p): C \rightarrow P$ by

$$
\bar{f}(p)(x)=E_{\Gamma}[f(\cdot, p, x)] .
$$

If $\gamma$ is chosen in $\Gamma$ and $p$ changes by $f(\gamma, p, x)$, then $\bar{f}(p)(x)$ is the expected change in $p$. The next lemma gives some properties of the function $\bar{f}$. It will be convenient to define the norms \|\| and $\left\||\||\right.$ on $\mathbf{L}^{d}$ in the same way as they were defined on $\mathbf{L}$. That is, for $\theta \in \mathbf{L}^{d}$,

$$
\begin{gathered}
k_{\theta}=\sup \{|\theta(x)-\theta(y)| /|x-y|: x \neq y \text { in } C\}, \\
\|\| \theta\|=\sup \{|\theta(x)| /(1+|x|): x \in C\}, \quad\| \theta \|=\max \left(k_{\theta},\||| \theta \mid\|\right) .
\end{gathered}
$$

Lemma 2.4. Let $p, q \in P$.

(i) $\bar{f}(p) \in \mathbf{L}^{d}$.

(ii) $\|\underline{f}(p)\| \leq k$.

(iii) $\|\bar{f}(p)\| \leq k$.

(iv) $|\bar{f}(p)(x)-\bar{f}(q)(y)| \leq k \cdot(|x-y|+|p-q|)$ for all $x, y \in C$.

(v) $\||\bar{f}(p)-\bar{f}(q) \|| \leq k \cdot|p-q|$.

Proof. We first prove (iv):

$$
\begin{aligned}
|\bar{f}(p)(x)-\bar{f}(q)(y)| & =\left|E_{\Gamma}[f(\cdot, p, x)-f(\cdot, q, y)]\right| \\
& \leq k \cdot(|x-y|+|p-q|) .
\end{aligned}
$$

This proves (iv). Taking $p=q$ in (iv), we have

$$
|\bar{f}(p)(x)-\bar{f}(p)(y)| \leq k \cdot|x-y|,
$$

so $\bar{f}(p)$ is Lipschitz on $C$ with bound $k$. Moreover, since $f$ has linear bound $k$ on $C$,

$$
|\bar{f}(p)(x)|=\left|E_{\Gamma}[f(\cdot, p, x)]\right| \leq k \cdot(1+|x|) .
$$

This shows that (i)-(iii) hold.

Taking $x=y$ in (iv), we have

$$
|(\bar{f}(p)-\bar{f}(q))(x)| \leq k \cdot|p-q| \leq k \cdot|p-q| \cdot(1+|x|),
$$

so (v) holds.

If $\theta=\left(\theta_{1}, \ldots, \theta_{d}\right) \in \mathbf{L}^{d}$, let $b(\theta)=\left(b\left(\theta_{1}\right), \ldots, b\left(\theta_{d}\right)\right)$.

Definition. We define the function $F: P \times \mathbf{B} \rightarrow R^{d}$ by $F(p, b)=b(\bar{f}(p))$. Note that $F$ is linear in $b$. In view of the next lemma, we call $F$ the expected price change function.

Lemma 2.5. In a finite exchange process, for all $t \in T$ and $\omega \in \Omega$ we have

$$
F(p(\omega, t), B(\omega, t)) \cdot \delta \cdot A \cdot \Delta t=E_{\Omega}\left[\Delta p(\cdot, t) \mid \mathbf{F}_{t}\right](\omega) .
$$

Proof. An agent $a \in A$ is chosen at time $t$, that is, $\omega_{A}(t+\Delta t)=a$, with probability $\Delta t$. If $a$ is chosen at time $t$, then the expected value of $\Delta p(\omega, t)$ is

$$
E_{\Gamma}[f(\cdot, p(\omega, t), X(\omega, a, t)) \cdot \delta]=\bar{f}(p(\omega, t))(X(\omega, a, t)) \cdot \delta
$$


Some $a \in A$ is chosen with probability $A \cdot \Delta t$. Averaging over $A$, we have

$$
\begin{aligned}
E_{\Omega}\left[\Delta p(\cdot, t) \mid \mathbf{F}_{t}\right](\omega) & =E_{A}[\bar{f}(p(\omega, t))(X(\omega, \cdot, t))] \cdot \delta \cdot A \cdot \Delta t \\
& =b_{X(\omega, \cdot, t)}[\bar{f}(p(\omega, t))] \cdot \delta \cdot A \cdot \Delta t \\
& =B(\omega, t)[\bar{f}(p(\omega, t))] \cdot \delta \cdot A \cdot \Delta t \\
& =F(p(\omega, t), B(\omega, t)) \cdot \delta \cdot A \cdot \Delta t .
\end{aligned}
$$

Definition. For each $j>0$, let $\mathbf{B}_{j}=\left\{b \in \mathbf{B}_{+}:\|b\| \leq j\right\}$. Since $\mathbf{B}_{+}$is closed in $\mathbf{B}, \mathbf{B}_{j}$ is closed in $\mathbf{B}$ for each $j$.

Lemma 2.6. (i) For all $b \in \mathbf{B}$ and $p \in P,|F(p, b)| \leq k \cdot\|b\|$.

(ii) For each $j>0, F$ is Lipschitz on the set $P \times \mathbf{B}_{j}$ with bound $j \cdot k$.

Proof. By Lemma 2.4(iii),

$$
|F(p, b)|=|b(\bar{f}(p))| \leq\|b\| \cdot\|\bar{f}(p)\| \leq\|b\| \cdot k .
$$

This proves (i). For (ii), let $p, q \in P$ and $b, c \in \mathbf{B}_{j}$. Then by Lemma 2.4,

$$
\begin{aligned}
|F(p, b)-F(q, c)| & =|b(\bar{f}(p))-c(\bar{f}(q))| \\
& \leq|b(\bar{f}(p)-\bar{f}(q))|+|(b-c)(\bar{f}(q))| \\
& \leq\|\| b|\|\cdot|\|\bar{f}(p)-\bar{f}(q) \mid\|+\|b-c\| \cdot\|\bar{f}(q)\| \\
& \leq\||| b|\|\cdot k \cdot|p-q|+\| b-c \| \cdot k \\
& \leq k \cdot(j \cdot|p-q|+\|b-c\|) \\
& \leq j \cdot k \cdot(|p-q|+\|b-c\|) .
\end{aligned}
$$

We next introduce an auxiliary function $\bar{g}$, which will be used to define the Lipschitz function $G: P \times \mathbf{B}_{+} \rightarrow \mathbf{B}$.

Definition. For each $\varphi \in \mathbf{L}$ and $p \in P$, define $\bar{g}(p, \varphi): C \rightarrow R$ by

$$
\bar{g}(p, \varphi)(x)=E_{\Gamma}[\varphi(x+g(\cdot, p, x))]-\varphi(x) .
$$

If $x$ changes by $g(\cdot, p, x)$, then $\bar{g}(p, \varphi)$ is the expected change in $\varphi(x)$.

Lemma 2.7. In a finite exchange process, for each $a, t$, and $\omega$ we have

$$
\begin{aligned}
& \bar{g}(p(\omega, t), \varphi)(X(\omega, a, t)) \cdot \Delta t \\
& \quad=E_{\Omega}\left[\varphi(X(\cdot, a, t+\Delta t))-\varphi(X(\cdot, a, t)) \mid \mathbf{F}_{t}\right](\omega) .
\end{aligned}
$$

Proof. The element $a$ is chosen at time $t$ with probability $\Delta t$. If $a$ is chosen, then the expected change in the value of $\varphi(X(\omega, a, t))$ from times $t$ to $t+\Delta t$ is $\bar{g}(p(\omega, t), \varphi)(X(\omega, a, t))$, and if $a$ is not chosen then $\varphi(X(\omega, a, t))$ remains unchanged.

Some properties of the function $\bar{g}$ are collected in the next lemma.

Lemma 2.8. Let $p, q \in P, x, y \in C$, and $\varphi, \theta \in \mathbf{L}$.

(i) $\bar{g}(p, \cdot)$ is a linear mapping from $\mathbf{L}$ into $\mathbf{L}$.

(ii) $\bar{g}(p, \mathbf{1})=0$, where $\mathbf{1}$ is the constant function with value 1 .

(iii) $\|\bar{g}(p, \varphi)\|\|\leq(k+2) \cdot\| \varphi \varphi \|$.

(iv) $\|\bar{g}(p, \varphi)\| \leq(k+2) \cdot\|\varphi\|$.

(v) $|\bar{g}(p, \varphi)(x)-\bar{g}(q, \varphi)(y)| \leq k_{\varphi} \cdot[k \cdot(|x-y|+|p-q|)+2 \cdot|x-y|]$.

(vi) ||$\left|\bar{g}(p, \varphi)-\bar{g}(q, \theta)\left\||| \leq(k+2) \cdot\left|\left\|\varphi-\theta|\||+k_{\varphi} \cdot k \cdot|p-q|\right.\right.\right.\right.$. 
Proof. (ii) is trivial. To prove (iii), let $x \in C$. Then

$$
\begin{aligned}
|\bar{g}(p, \varphi)(x)| & =\left|E_{\Gamma}[\varphi(x+g(\cdot, p, x))]-\varphi(x)\right| \\
& \leq\left|E_{\Gamma}[\varphi(x+g(\cdot, p, x))]\right|+|\varphi(x)| \\
& \leq|\| \varphi||| \cdot(2+2|x|+k \cdot(1+|x|)) \\
& \leq|\|\varphi \mid\| \cdot(k+2) \cdot(1+|x|),
\end{aligned}
$$

so (iii) holds. We prove (v) next and use (v) to prove (i), (iv), and (vi).

$$
\begin{aligned}
\mid \bar{g}(p & , \varphi)(x)-\bar{g}(q, \varphi)(y) \mid \\
& =\left|E_{\Gamma}[\varphi(x+g(\cdot, p, x))-\varphi(y+g(\cdot, q, y))]-(\varphi(x)-\varphi(y))\right| \\
& \leq\left|E_{\Gamma}[\varphi(x+g(\cdot, p, x))-\varphi(y+g(\cdot, q, y))]\right|+|\varphi(x)-\varphi(y)| \\
& \leq k_{\varphi} \cdot\left|E_{\Gamma}[(g(\cdot, p, x))-(g(\cdot, q, y))]\right|+2 \cdot k_{\varphi} \cdot|x-y| \\
& \leq k_{\varphi} \cdot[k \cdot(|x-y|+|p-q|)+2 \cdot|x-y|] .
\end{aligned}
$$

This proves (v). Applying (v) with $p=q$, we see that $\bar{g}(p, \varphi)$ has Lipschitz bound $k_{\varphi} \cdot(k+2)$, and in view of (iii), (iv) holds. Therefore, $\bar{g}(p, \varphi) \in L$, and (i) holds. Finally, we apply (i) $-(v)$ to prove (vi):

$$
\begin{aligned}
\| \mid \bar{g}(p & , \varphi)-\bar{g}(q, \theta)\|\| \\
& \leq\||| \bar{g}(p, \varphi)-\bar{g}(q, \varphi) \mid\|+\|\bar{g}(q, \varphi)-\bar{g}(q, \theta)\| \| \\
& \leq\||\bar{g}(p, \varphi)-\bar{g}(q, \varphi)\|\mid+\| \bar{g}(q, \varphi-\theta)\|\| \\
& \leq \sup \{|\bar{g}(p, \varphi)(x)-\bar{g}(q, \varphi)(x)|: x \in C\}+\|\mid \bar{g}(q, \varphi-\theta)\| \| \\
& \leq k_{\varphi} \cdot k \cdot|p-q|+(k+2) \cdot\|\varphi-\theta\| \| . \quad
\end{aligned}
$$

Definition. Let $G$ be the function $G: P \times \mathbf{B} \rightarrow \mathbf{B}$ defined by $G(p, b)(\varphi)=$ $b(\bar{g}(p, \varphi))$. In view of the next lemma, we call $G$ the expected state change function. Note that $G$ is inear in $b$.

Lemma 2.9. In a finite exchange process, for each $\omega \in \Omega$ and $t \in T$ we have

$$
G(p(\omega, t), B(\omega, t)) \cdot \Delta t=E_{\Omega}\left[B(\cdot, t+\Delta t)-B(\cdot, t) \mid \mathbf{F}_{t}\right](\omega) .
$$

Proof. Let $\varphi \in \mathbf{L}$. Then

$$
\begin{aligned}
& E_{\Omega}\left[B(\cdot, t+\Delta t)-B(\cdot, t) \mid \mathbf{F}_{t}\right](\omega)(\varphi) \\
& =E_{\Omega}\left[(B(\cdot, t+\Delta t)-B(\cdot, t))(\varphi) \mid \mathbf{F}_{t}\right](\omega) \\
& =E_{\Omega}\left[\left(b_{X(\cdot, \cdot, t+\Delta t)}-b_{X(\cdot, \cdot, t)}\right)(\varphi) \mid \mathbf{F}_{t}\right](\omega) \\
& =E_{\Omega}\left[E_{A}[\varphi(X(\cdot, \cdot, t+\Delta t))-\varphi(X(\cdot, \cdot, t))] \mid \mathbf{F}_{t}\right](\omega) \\
& =E_{A}\left[E_{\Omega}\left[\varphi(X(\cdot, \cdot, t+\Delta t))-\varphi(X(\cdot, \cdot, t)) \mid \mathbf{F}_{t}\right](\omega)\right] \\
& =E_{A}[\bar{g}(p(\omega, t), \varphi)(X(\omega, \cdot, t)) \cdot \Delta t] \\
& =b_{X(\omega, \cdot, t)}(\bar{g}(p(\omega, t), \varphi)) \cdot \Delta t \\
& =B(\omega, t)(\bar{g}(p(\omega, t), \varphi)) \cdot \Delta t \\
& =G(p(\omega, t), B(\omega, t)) \cdot \Delta t(\varphi) \text {. }
\end{aligned}
$$

The next lemma gives some properties of the function $G$.

Lemma 2.10. Let $b \in \mathbf{B}$ and $p \in P$.

(i) $G(p, b) \in \mathbf{B}$.

(ii) $\|G(p, b)\| \leq(k+2) \cdot\|b\|$. 
(iii) $\||G(p, b)\||\leq(k+2) \cdot\|\| b|\|$.

(iv) $G(p, b)(\mathbf{1})=0$.

(v) For each $j \geq 1, G$ is uniformly Lipschitz on $P \times \mathbf{B}_{j}$ with Lipschitz bound $j \cdot(k+2)$.

(vi) For all $\alpha \in[0,1]$, if $b \in \mathbf{M}$ then $b+\alpha \cdot G(p, b) \in \mathbf{M}$.

Proof. Since $\bar{g}(p, \varphi)$ is linear in $\varphi, G(p, b)(\varphi)$ is linear in $\varphi$. Let $\varphi \in \mathbf{L}$ and $\|\varphi\| \leq 1$. Then, by Lemma 2.8 (iv),

$$
\|\bar{g}(p, \varphi)\| \leq(k+2) \cdot\|\varphi\|
$$

and

$$
\begin{aligned}
|G(p, b)(\varphi)| & =|b(\bar{g}(p, \varphi))| \leq\|b\| \cdot\|\bar{g}(p, \varphi)\| \\
& \leq\|b\| \cdot(k+2) \cdot\|\varphi\| \leq(k+2) \cdot\|b\| .
\end{aligned}
$$

This proves (i) and (ii).

Now let $\|\mid \varphi\| \|=1$. By Lemma 2.8(iii),

$$
\begin{aligned}
|G(p, b)(\varphi)| & =|b(\bar{g}(p, \varphi))| \leq\||| b|||\cdot||\bar{g}(p, \varphi)|\| \mid \\
& \leq|||b||| \cdot(k+2) \cdot\||| \varphi||=(k+2) \cdot\||| b|| .
\end{aligned}
$$

This proves (iii). (iv) is immediate from Lemma 2.8 (ii).

To prove (v), let $j \geq 1, b, c \in \mathbf{B}_{j}$, and $p, q \in P$. Let $\varphi \in \mathbf{L}$ with $\|\varphi\|=1$. Then, using Lemma 2.8(vi),

$$
\begin{aligned}
\mid G(p & , b)(\varphi)-G(q, c)(\varphi)|=| b(\bar{g}(p, \varphi))-c(\bar{g}(q, \varphi)) \mid \\
& \leq|b(\bar{g}(p, \varphi)-\bar{g}(q, \varphi))|+|(b-c)(\bar{g}(q, \varphi))| \\
& \leq|\|b|\|\cdot|\|\bar{g}(p, \varphi)-\bar{g}(q, \varphi)\||+\| b-c\|\cdot\| \bar{g}(q, \varphi) \| \\
& \leq j \cdot k \cdot k_{\varphi} \cdot|p-q|+\|b-c\| \cdot(k+2) \cdot\|\varphi\| \\
& \leq j \cdot(k+2) \cdot(|p-q|+\|b-c\|) .
\end{aligned}
$$

This proves $(\mathrm{v})$.

It remains to prove (vi). Let $\mu$ be the probability measure on $C$ such that for each Borel set $U \subset C, \mu(U)$ is the probability of the set $\{(\gamma, x) \in$ $\Gamma \times C: x+g(\gamma, p, x) \in U\}$ with respect to the product of the counting measure on $\Gamma$ and the measure $b$ on $C$. Then $\mu$ is a Borel probability measure on $C$, and since $g$ is linearly bounded on $C, \mu \in \mathbf{M}$. For any $\varphi \geq 0$ in $\mathbf{L}$, $\mu(\varphi)=b(\theta)$, where $\theta(x)=E_{\Gamma}[\varphi(x+g(\cdot, p, x))]$. It follows that

$$
\begin{aligned}
(b+\alpha \cdot G(p, b))(\varphi) & =b(\varphi)+\alpha \cdot b(\bar{g}(p, \varphi)) \\
& =b(\varphi)+\alpha \cdot b\left(E_{\Gamma}[\varphi(x+g(\cdot, p, x))-\varphi(x)]\right) \\
& =(1-\alpha) \cdot b(\varphi)+\alpha \cdot b(\theta)=(1-\alpha) \cdot b(\varphi)+\alpha \cdot \mu(\varphi) .
\end{aligned}
$$

Therefore

$$
b+\alpha \cdot G(p, b)=(1-\alpha) \cdot b+\alpha \cdot \mu,
$$

and hence $b+\alpha \cdot G(p, b)$ is a convex combination of elements of $\mathbf{M}$ and thus belongs to $\mathbf{M}$ by Lemma 2.2 .

Our definitions of $F(p, b)$ and $G(p, b)$ are meaningful even when $b \in \mathbf{B}$ instead of $b \in \mathbf{B}_{+}$. However, the proofs that $F$ and $G$ are Lipschitz on $P \times \mathbf{B}_{+}$ use the triple norm and do not carry over to $P \times \mathbf{B}$.

Theorem 2.1 follows from Lemmas 2.5, 2.6, 2.9, and 2.10. 


\section{SOLUTIONS AND APPROXIMATIONS}

In this section we shall consider the differential equation $b^{\prime}(t)=G(p(t), b(t))$ which describes the path of the "expected commodity state." We shall show in Theorem 3.7 that if $b(0)$ is a measure supported by a bounded subset of $C$, and $p(t)$ has the form $H(t, b(t))$, where $H$ is Lipschitz, then this equation has a unique solution $b(t) \in \mathbf{M}$ for all $t \geq 0$. The difficulty is that the set $\mathbf{M}$ with the \|\| -norm is not compact, and we need to find compact subsets of $\mathbf{M}$ which contain approximate solutions.

Another difficulty which will arise in the next section is that the spaces $(\mathbf{L},\|\|)$ and $(\mathbf{B},\|\|)$ are not separable. At the end of this section we shall prove a lemma concerning relatively dense finite subsets of $\mathbf{L}$ which will serve to some extent as a substitute for separability.

We begin by introducing subspaces of $\mathbf{L}$ and $\mathbf{B}$ which come from bounded subsets of the commodity space $C$. Let $q$ be a positive real number. For each $x \in C$, we define

$$
x^{\wedge} q= \begin{cases}x & \text { if }|x| \leq q, \\ x \cdot q /|x| & \text { if }|x| \geq q .\end{cases}
$$

For each $x \in C, \varphi \in \mathbf{L}$, and $b \in \mathbf{B}$, we define

$$
\left(\varphi^{\wedge} q\right)(x)=\varphi\left(x^{\wedge} q\right), \quad\left(b^{\wedge} q\right)(\varphi)=b\left(\varphi^{\wedge} q\right) .
$$

Moreover, we let

$$
x^{\triangle} q=x-x^{\wedge} q, \quad \varphi^{\triangle} q=\varphi-\varphi^{\wedge} q, \quad b^{\triangleright} q=b-b^{\wedge} q
$$

and

$$
\mathbf{L}^{\wedge} q=\{\widehat{\varphi} q: \varphi \in \mathbf{L}\}, \quad \mathbf{B}^{\wedge} q=\left\{b^{\wedge} q: b \in \mathbf{B}\right\} .
$$
tion.

In the next two lemmas we state some elementary properties of this trunca-

Lemma 3.1. Let $x, y \in C$ and $\varphi, \theta \in \mathbf{L}$. For all positive real numbers $q$,

(i) $\left|x^{\wedge} q-y^{\wedge} q\right| \leq|x-y|$.

(ii) $|x \sim q|=\min (|x|, q)$.

(iii) The mapping $\varphi \rightarrow \varphi^{\wedge} q$ is linear on $\mathbf{L}$.

(iv) If $q \leq r$ and $u$ belongs to either $C, \mathbf{L}$, or $\mathbf{B}$, then $\left(u^{\wedge} q\right)^{\wedge} r=u^{\wedge} q=$ $\left(u^{\wedge} r\right)^{\wedge} q$.

(v) $k_{\varphi^{\wedge} q},\left\|\mid \varphi^{\wedge} q\right\| \|$, and $\left\|\varphi^{\wedge} q\right\|$ are nondecreasing in $q$.

(vi) As $q \rightarrow \infty$, we have $k_{\varphi^{\wedge} q} \rightarrow k_{\varphi},\left\|\mid \varphi^{\wedge} q\right\|\|\rightarrow\| \varphi\|\|$, and $\left\|\varphi^{\wedge} q\right\| \rightarrow\|\varphi\|$.

(vii) $\mathbf{L}^{\wedge} q$ is a linear subspace of $\mathbf{L}$.

Proof. Parts (i)-(v) are elementary. An easy computation shows that for each $q, k_{\varphi^{\wedge} q} \leq k_{\varphi}$. To prove (vi), we suppose $r<k_{\varphi}$ and show that for some $q$, $r<k_{\varphi \wedge}^{\wedge}$. For some $x \neq y$,

$$
|\varphi(x)-\varphi(y)| /|x-y|>r .
$$

Let $q$ be such that $|x|<q$ and $|y|<q$. Then $x=x^{\wedge} q$ and $y=y^{\wedge} q$, so

$$
\begin{aligned}
r & <\left|\varphi\left(x^{\wedge} q\right)-\varphi\left(y^{\wedge} q\right)\right| /|x-y| \\
& =\left|\left(\varphi^{\wedge} q\right)(x)-\left(\varphi^{\wedge} q\right)(y)\right| /|x-y|,
\end{aligned}
$$

and $r<k_{\varphi^{\wedge} q}$. The proofs for the norms \|\| and $\|\mid\|$ are similar. Part (vii) follows from (iii)-(vi). 
Notice that $\left\|\operatorname{abs}^{\triangle} q\right\| \|=1$ and $\operatorname{abs}^{\triangle} q$ has Lipschitz bound 1 for each $q$. Thus abs $\widehat{q}$ does not approach abs in the Lipschitz norm and does not approach abs in the |||||| -norm as $q \rightarrow \infty$.

Lemma 3.2. Let $q$ be a positive real number.

(i) The mapping $b \mapsto b^{\wedge} q$ is linear on $\mathbf{B}$.

(ii) For each $b \in \mathbf{B},\left\|b^{\wedge} q\right\| \|$ and $\left\|b^{\wedge} q\right\|$ are nondecreasing in $q$.

(iii) For each $b \in \mathbf{B},\left\|b^{\wedge} q\right\|\|\leq\||| b\|\mid\|$ and $\left\|b^{\wedge} q\right\| \leq\|b\|$.

(iv) $(\mathbf{B} \wedge q,\|\|)$ is a Banach subspace of $(\mathbf{B},\|\|)$.

(v) If $b \in \mathbf{B}_{+}$, then $b^{\wedge} q \in \mathbf{B}_{+}$and $\left\|b^{\wedge} q\right\| \| \leq(\mathbf{1}) \cdot(1+q)$.

(vi) If $b \in \mathbf{M}$, then $b^{\wedge} q \in \mathbf{M}$ and $\left\|b^{\wedge} q\right\| \| \leq 1+q$.

(vii) A measure $b \in \mathbf{M}$ belongs to $\mathbf{B}^{\wedge} q$ if and only if the set $\{x \in C:|x| \leq q\}$ has $b$-measure one.

Proof. This follows easily from Lemma 3.1. As an illustration, we prove (ii) for $\left\|b^{\wedge} q\right\|$. We first observe that

$$
\left\|b^{\wedge} q\right\|=\sup \{|b(\varphi)| /\|\varphi\|: 0 \neq \varphi \in \mathbf{L} \prec q\},
$$

because

$$
\begin{aligned}
\sup \{ & \left.|b(\varphi)| /\|\varphi\|: 0 \neq \varphi \in \mathbf{L}^{\wedge} q\right\} \\
& =\sup \left\{\left|b\left(\varphi^{\wedge} q\right)\right| /\|\varphi\|: 0 \neq \varphi \in \mathbf{L}^{\wedge} q\right\} \\
& \leq \sup \left\{\left|b\left(\varphi^{\wedge} q\right)\right| /\|\varphi\|: 0 \neq \varphi \in \mathbf{L}\right\} \\
& =\sup \left\{\left|\left(b^{\wedge} q\right)(\varphi)\right| /\|\varphi\|: 0 \neq \varphi \in \mathbf{L}\right\}=\left\|b^{\wedge} q\right\| \\
& \leq \sup \left\{\left|b\left(\varphi^{\wedge} q\right)\right| /\left\|\varphi^{\wedge} q\right\|: 0 \neq \varphi^{\wedge} q, \varphi \in \mathbf{L}\right\} \\
& =\sup \left\{|b(\varphi)| /\|\varphi\|: 0 \neq \varphi \in \mathbf{L}^{\frown} q\right\} .
\end{aligned}
$$

If $q \leq r$, then $\mathbf{L}^{\wedge} q \subset \mathbf{L}^{\wedge} r$, and it follows that $\left\|b^{\wedge} q\right\| \leq\left\|b^{\wedge} r\right\|$.

Example. The analogue of Lemma 3.1(vi) does not hold for $b \in \mathbf{B}_{+}$; there exist $b \in \mathbf{B}_{+}$such that $\left\|b^{\wedge} q\right\|$ does not approach $\|b\|$ as $q$ approaches $\infty$. Let $x_{n}$, $n \in \mathbf{N}$, be a sequence of points in $C$ such that $\left|x_{n}\right| \rightarrow \infty$, let $U$ be a free ultrafilter over $\mathbf{N}$, and let $b$ be the functional $b(\varphi)=U-\operatorname{limit}\left(\varphi\left(x_{n}\right) /\left|x_{n}\right|: n \in\right.$ $\mathbf{N})$. Or, equivalently, let $x$ be an infinite element of ${ }^{*} C$ in a nonstandard universe and let $b(\varphi)$ be the standard part of ${ }^{*} \varphi(x) /|x|$. Then $b \in \mathbf{B}_{+}$and $\|b\|=1$, but $\left\|b^{\wedge} q\right\|=0$ for each finite $q$.

Our next lemma shows that for a measure $b \in \mathbf{M},\left\|b^{\wedge} q\right\|$ approaches $\|b\|$ as $n \rightarrow \infty$, and in fact $b^{\wedge} q$ approaches $b$ in the $\||\|| \mid-$ norm as $q \rightarrow \infty$.

Lemma 3.3. Let $b \in \mathbf{M}$. For each $q \geq 3$,

$$
\left(b^{\triangle} q\right)(\text { abs }) \leq\left\|b^{\triangle} q\right\| \leq\left\|b^{\triangle} q\right\| \mid \leq 4\left(b^{\triangle}(q / 3)\right)(\text { abs })
$$

and $\left\|b^{\triangle} q\right\| \| \rightarrow$ as $q \rightarrow \infty$.

Proof. $b$ is positive definite and abs $\widehat{q}$ increases monotonically to abs everywhere on $C$ as $q \rightarrow \infty$, so

$$
\left(b^{\triangle} q\right)(\mathrm{abs})=\left|\left(b^{\triangle} q\right)(\mathrm{abs})\right|=\left|b\left(\mathrm{abs}^{\triangle} q\right)\right|
$$

and $\left|b\left(\operatorname{abs}^{\triangle} q\right)\right| \rightarrow 0$ as $q \rightarrow \infty$. Since $\|\mathrm{abs}\|=1$,

$$
\left(b^{\triangle} q\right)(\text { abs }) \leq\left\|b^{\triangle} q\right\| \leq\left\|b^{\triangle} q\right\| \| .
$$


To prove the other inequality, let $\varphi \in \mathbf{L}$ and $\|\varphi\| \| \leq 1$, and let $1 \leq r=q / 3$. Then

$$
\begin{aligned}
\left|\left(b^{\triangle} q\right)(\varphi)\right| & =\left|b(\varphi)-b\left(\varphi^{\wedge} q\right)\right|=\left|\int \varphi(x)-\varphi\left(x^{\wedge} q\right) d b\right| \\
& =\left|\int_{|x| \geq q} \varphi(x)-\varphi\left(x^{\wedge} q\right) d b\right| \leq \int_{|x| \geq q}|\varphi(x)|+\left|\varphi\left(x^{\wedge} q\right)\right| d b \\
& \leq \int_{|x| \geq q}(\operatorname{abs}(x)+q+1) d b=\int_{|x| \geq q}(\operatorname{abs}(x)-(r+1))+(4 r+2) d b \\
& \leq 4 \int_{|x| \geq q}(\operatorname{abs}(x)-(r+1)) d b \leq 4\left(b\left(\operatorname{abs}^{\Delta} r\right)\right),
\end{aligned}
$$

as required.

It follows from Lemma 3.3 that for $b \in \mathbf{M},\left\|b^{\wedge} q\right\| \rightarrow\|b\|$ and $\left\|b^{\triangleleft} q\right\| \rightarrow 0$ as $q \rightarrow \infty$. We shall need more detailed information about the convergence of $b^{-} q$ to $b$.

Definition. Let $J$ be a positive real number. We define $\mathbf{M}(J)=\{b \in \mathbf{M}:\|b\| \leq$ $J$ and for all $\left.q \geq 1,\left\|b^{\triangle} q\right\| \leq J / q\right\}$.

Example. If $X$ maps $A$ into the ball of radius $r$, then $b_{X} \in \mathbf{M} \cap \mathbf{B} \frown r$. If $b \in \mathbf{M} \cap \mathbf{B} \wedge r$ and $r \geq 1$, then $b \in M(J)$, where $J=2 r \cdot(r+1)$.

Lemma 3.4. For each $J \geq 1$, the set $\mathbf{M}(J)$ is convex and compact in $(\mathbf{B},\|\|)$. Proof. It is easy to check that $\mathbf{M}(J)$ is convex. To show that $\mathbf{M}(J)$ is compact, we take a nonstandard universe and use the result of Robinson (see [SB]) that a set $Y$ in a metric space is compact if and only if every element of ${ }^{*} Y$ is infinitely close to a standard element of $Y$. We shall prove that every $B \in$ ${ }^{*} \mathbf{M}(J)$ is infinitely close to a standard element of $\mathbf{M}(J)$ with respect to the \|\| -norm. Given $B \in{ }^{*} \mathbf{M}(J)$, let $\mu$ be the Loeb measure on ${ }^{*} C$ induced by $B$ and let $b$ be the Borel measure on $C$ given by $b(U)=\mu\left(\mathrm{st}^{-1} U\right)$. When $|x| \geq q+1 \geq 2$, we have $\left(\operatorname{abs}^{\triangle} q\right)(x) \geq 1$ and hence, by Lemma 3.3,

$$
\mu\{x:|x| \geq q+1\} \leq B\left(\operatorname{abs}^{\triangle} q\right) \leq\left\|B^{\triangle} q\right\| \leq J / q,
$$

so $\mu$ is supported by $\mathrm{st}^{-1}(C)$ and $\mu\left(\mathrm{st}^{-1}(C)=1=b(C)\right.$. Moreover,

$$
b(\mathrm{abs}) \approx B\left({ }^{*} \mathrm{abs}\right) \leq J .
$$

Thus $b$ is a probability measure on $C$ for which the expected value of $|x|$ is finite, and hence $b \in \mathbf{M}$. Then for all $\varphi \in \mathbf{L}, b(\varphi) \approx B\left({ }^{*} \varphi\right)$ and

$$
\left|\left(b^{\triangle} q\right)(\varphi)\right|=\left|b\left(\varphi^{\triangle} q\right)\right| \approx\left|B\left({ }^{*} \varphi^{\triangle} q\right)\right|=\left|\left(B^{\triangle} q\right)\left({ }^{*} \varphi\right)\right| \leq J \cdot\|\varphi\| / q,
$$

so $\left\|b^{\triangle} q\right\| \leq J / q$. Therefore $b \in \mathbf{M}(J)$.

We must show that $\left\|B-{ }^{*} b\right\| \approx 0$. For each finite $q$,

$$
\begin{aligned}
\left\|B-{ }^{*} b\right\| & \leq\left\|B^{\triangle} q\right\|+\left\|B^{\wedge} q-{ }^{*}\left(b^{\frown} q\right)\right\|+\left\|b^{\triangle} q\right\| \\
& \leq 2 J / q+\left\|B^{\wedge} q-{ }^{*}\left(b^{\frown} q\right)\right\| .
\end{aligned}
$$

Let $\varphi \in{ }^{*} \mathbf{L}$ and $\|\varphi\| \leq 1$. Then for each finite $q,\left\|\varphi^{\wedge} q\right\| \leq 1$. Let $\theta$ be the standard function from $C$ into $R$ defined by $\theta(\operatorname{st}(x))=\operatorname{st}\left(\varphi\left(x^{\wedge} q\right)\right)$. Then $\theta \in \mathbf{L}^{\wedge} q$ and $\|\theta\| \leq 1$. For all $x \in{ }^{*} C$,

$$
\left(\varphi^{\wedge} q\right)(x)=\varphi(x \prec q) \approx{ }^{*} \theta(x \prec q)={ }^{*} \theta(x),
$$


so $\left\|\mid \varphi^{\wedge} q-{ }^{*} \theta\right\| \| \approx$. Therefore

$$
\begin{aligned}
& \left|\left(B^{\wedge} q-{ }^{*}\left(b^{\frown} q\right)\right)(\varphi)\right|=\left|B\left(\varphi^{\wedge} q\right)-{ }^{*} b\left(\varphi^{\wedge} q\right)\right| \\
& \leq\left|B\left(\varphi^{\wedge} q-{ }^{*} \theta\right)\right|+\left|B\left({ }^{*} \theta\right)-b(\theta)\right|+\left|{ }^{*} b\left({ }^{*} \theta-\varphi^{\wedge} q\right)\right| \\
& \leq(|||B|||+|||b|||) \cdot||\left|\varphi^{-} q-{ }^{*} \theta\right|||+\left|B\left({ }^{*} \theta\right)-b(\theta)\right| \\
& \leq 2 J \cdot\left\|\left|\varphi^{\wedge} q-{ }^{*} \theta \|\right|+\left|B\left({ }^{*} \theta\right)-b(\theta)\right| .\right.
\end{aligned}
$$

Since $B\left({ }^{*} \theta\right) \approx b(\theta)$, we conclude that

$$
\left|\left(B^{\wedge} q-{ }^{*}\left(b^{\frown} q\right)\right)(\varphi)\right| \approx 0,
$$

and therefore $\left\|B-{ }^{*} b\right\| \approx 0$.

Remark. The above proof can readily be extended to show that for every decreasing function $J:[1, \infty) \rightarrow[0, \infty)$ such that $J(q) \rightarrow 0$ as $q \rightarrow \infty$, the set

$$
\left\{b \in \mathbf{M}:\|b\| \leq J(1) \text { and for all } q \geq 1,\left\|b^{\triangle} q\right\| \leq J(q)\right\}
$$

is convex and compact.

Lemma 3.5. Let the function $g: \Gamma \times P \times C \rightarrow C$ be uniformly Lipschitz on $P \times C$ with bound $k$ and linearly bounded on $C$ with bound $k$, and let $G$ be the associated function $G: P \times \mathbf{B} \rightarrow \mathbf{B}$. Then there is a constant $c_{1}$ depending only on $k$ such that whenever $b \in \mathbf{M}(J)$, where $J>0, p \in P$, and $\alpha \in[0,1]$,

$$
b+\alpha \cdot G(p, b) \in \mathbf{M}\left(\left[1+\alpha \cdot c_{1}\right] \cdot J\right) .
$$

Proof. By Lemma 2.10(vi), $b+\alpha \cdot G(p, b)$ belongs to M. By 2.10(ii),

$$
\begin{aligned}
\|b+\alpha \cdot G(p, b)\| & \leq\|b\|+\alpha \cdot\|G(p, b)\| \\
& \leq\|b\| \cdot[1+\alpha \cdot(k+2)] \leq[1+\alpha \cdot(k+2)] \cdot J .
\end{aligned}
$$

From the proof of Lemma 2.10(vi),

$$
b+\alpha \cdot G(p, b)=(1-\alpha) \cdot b+\alpha \cdot \mu,
$$

where $\mu \in \mathbf{M}$ is defined by $\mu(\varphi)=b(\theta), \theta(x)=E_{\Gamma}[\varphi(x+g(\cdot, p, x))]$. Since $g$ has linear bound $k$, for each $\gamma \in \Gamma$ we have

$$
|g(\gamma, p, x)| \leq(|x|+1) \cdot k .
$$

Therefore whenever $q \geq 2 k, k \geq 1$, and $|x+g(\gamma, p, x)| \geq q$, we have

$$
|x| \geq(q-k) /(k+1) \geq q /(4 k) .
$$

By the argument used in the proof of Lemma 3.3, when $q \geq 12 k$ and $r=$ $q / 12 k$, we have

$$
\begin{aligned}
\left\|\mu^{\triangle} q\right\| & \leq\left\|\mid \mu^{\triangle} q\right\| \| \leq \int_{|y| \geq q} \operatorname{abs}(y)+q+1 d \mu \\
& \leq \int_{|x| \geq q / 4 k} E_{\Gamma}[\operatorname{abs}(x+g(\cdot, p, x))]+q+1 d b \\
& \leq(k+1) \cdot \int_{|x| \geq q / 4 k} \operatorname{abs}(x)+q+1 d b \leq 4(k+1) \cdot\left(b^{\triangle} r\right)(\text { abs }) \\
& \leq 4(k+1) \cdot\left\|b^{\triangle} r\right\| \leq 4(k+1) \cdot J / r=4(k+1) \cdot(12 k) \cdot J / q .
\end{aligned}
$$


On the other hand, whenever $1 \leq q \leq 12 k$,

$$
\begin{aligned}
\left\|\mu^{\triangle} q\right\| & \leq\|\mu\|+\left\|\mu^{\wedge} q\right\| \leq 2 \cdot\|\mu\| \\
& \leq 2(k+1) \cdot\|b\| \leq 2(k+1) \cdot J \\
& =2(k+1) \cdot(12 k) \cdot J / 12 k \leq 2(k+1) \cdot(12 k) \cdot J / q .
\end{aligned}
$$

Let $b_{1}=b+\alpha \cdot G(p, b)=(1-\alpha) \cdot b+\alpha \cdot \mu$. Then

$$
\begin{aligned}
\left\|b_{1}{ }^{\triangle} q\right\| & \leq(1-\alpha) \cdot J / q+\alpha \cdot\left\|\mu^{\triangle} q\right\| \\
& \leq(1-\alpha) \cdot J / q+\alpha \cdot 4(k+1) \cdot(12 k) \cdot J / q \\
& =[1+\alpha \cdot(4(k+1) \cdot(12 k)-1)] \cdot J / q .
\end{aligned}
$$

Thus $b_{1} \in \mathbf{M}\left(\left[1+\alpha \cdot c_{1}\right] \cdot J\right)$, where $c_{1}=4(k+1) \cdot(12 k)-1$.

Corollary 3.6. Let the function $G$ and constant $c_{1}$ be as in Lemma 3.5, and let $0<\Delta t \leq 1$ and $T=\{n \cdot \Delta t: n \in \mathbf{N}\}$. Let $b(0) \in M(J)$, where $J>0$, and let $H$ be a function mapping $T \times \mathbf{M}$ into the price space $P$. Define $b(t), t \in T$, by the difference equation

$$
b(t+\Delta t)=b(t)+G(H(t, b(t)), b(t)) \cdot \Delta t .
$$

Then for each $t \in T$,

$$
b(t) \in \mathbf{M}\left(\exp \left(c_{1} \cdot t\right) \cdot J\right) .
$$

Proof. Apply Lemma 3.5 repeatedly with $\alpha=\Delta t$.

Theorem 3.7. Let $G$ and $c_{1}$ be as in Lemma 3.5, let $b_{0} \in \mathbf{M}(J)$, where $J>0$, and let $H:[0, \infty) \times \mathbf{B}_{+} \rightarrow P$.

(i) (Existence) If $H$ is continuous then the initial value problem

$$
b(0)=b_{0}, \quad b^{\prime}(t)=G(H(t, b(t)), b(t))
$$

has a solution $b$ such that $b(t) \in M\left(\exp \left(c_{1} \cdot t\right) \cdot J\right)$ for each $t \in[0, \infty)$.

(ii) (Uniqueness) If $H$ is uniformly Lipschitz on $[0, \infty) \times S$ where $S \subseteq \mathbf{B}_{+}$, then problem (1) has at most one solution $b:[0, t) \rightarrow S$ for each $t \leq \infty$.

Proof. (i) The method of the classical Peano existence proof with approximations by difference equations goes through. Use Corollary 3.6 to show that the solutions of the difference equations belong to $\mathbf{M}\left(\exp \left(c_{1} \cdot t\right) \cdot J\right)$, and use the compactness from Lemma 3.4 to show that as $\Delta t \rightarrow 0$ they approach a solution of (1) which belongs to $\mathbf{M}\left(\exp \left(c_{1} \cdot t\right) \cdot J\right)$.

(ii) This is proved in the usual way by finding an exponential bound for the difference between two solutions.

In the above theorem, the existence holds even if the function $H$ is defined only on $[0, \infty) \times \mathbf{M}$.

We now prove a companion result (Proposition 3.9) concerning the commodity state $B(\omega, t)$ for a finite exchange process, which will be needed in $\S 5$.

Lemma 3.8. Let $(p, X)$ be a finite exchange process with Lipschitz bound $k$ and the initial allocation $X(\cdot, 0)$ uniformly bounded by $k$. Let $a \in A$ and $t \in T$, and let $n(\omega, a, t)$ be the number of times that agent $a$ is chosen during the time interval $0 \leq u \leq t$.

(i) For each $n \in \overline{\mathbf{N}}$, the probability that $n(\omega, a, t)=n$ is at most $t^{n} / n !$. 
(ii) $\operatorname{abs}(X(\omega, a, t)) \leq(k+1)^{n(\omega, a, t)+1}$.

Proof. (i) Let $D=t / \Delta t$. Then

$$
\begin{aligned}
P_{\Omega}[n(\cdot, a, t)=n] & \leq\left(\begin{array}{l}
D \\
n
\end{array}\right) \cdot(\Delta t)^{n}=\left(D ! \cdot(\Delta t)^{n} /(n ! \cdot(D-n) !)\right. \\
& \leq(D \cdot \Delta t)^{n} / n !=t^{n} / n ! .
\end{aligned}
$$

(ii) We have $|X(a, 0)| \leq k$, so $\operatorname{abs}(X(\omega, a, 0)) \leq k+1$. If $a$ is chosen at time $u$, then

$$
\begin{aligned}
\operatorname{abs}(X(\omega, a, u+\Delta t)) & =|X(\omega, a, u+\Delta t)|+1 \\
& =|X(\omega, a, u)+g(\gamma, p(\omega, u), X(\omega, a, u))| \\
& \leq|X(\omega, a, u)|+1+k \cdot(|X(\omega, a, u)|+1) \\
& =(k+1) \cdot \operatorname{abs}(X(\omega, a, u)) .
\end{aligned}
$$

It follows by induction on $n(\omega, a, u)$ that for each $u \leq t$,

$$
\operatorname{abs}(X(\omega, a, u)) \leq(k+1)^{n(\omega, a, u)+1} \text {. }
$$

Proposition 3.9. Let $(p, X)$ be a finite exchange process with Lipschitz bound $k$, and suppose that the initial allocation $X(\omega, 0)$ is also uniformly bounded by $k$. Let $t \in T$. Then

(i) $E_{\Omega}[\max (\|B(\cdot, u)\|: u \leq t)] \leq(k+1) \cdot e^{(k+1) \cdot t}$.

(ii) There is a constant $c$ which depends only on $k$ such that for each $1 \leq$ $t \in T$ and $z \geq 1$,

$$
P_{\Omega}\left[\max (\|B(\cdot, u)\|: u \leq t) \geq z \cdot e^{c \cdot t}\right] \leq 1 /\left(z^{2} \cdot A\right) .
$$

Proof. (i) Let $n(\omega, a, t)$ be as in Lemma 3.8. Observe that $n(\omega, a, u)$ is nondecreasing in $u$. By Lemmas 2.3 and 3.8(ii),

$$
\begin{aligned}
\max (\|B(\omega, u)\|: u \leq t) & =\max (B(\omega, u)(\mathrm{abs}): u \leq t) \\
& =E_{A}[\max (\operatorname{abs}(X(\omega, \cdot, u)): u \leq t)] \\
& \leq E_{A}\left[(k+1)^{n(\omega, \cdot, t)+1}\right] .
\end{aligned}
$$

By Lemma 3.8(i), for each $a \in A$,

$$
E_{\Omega}\left[(k+1)^{n(\cdot, a, t)+1}\right] \leq \sum_{n=0}^{\infty}(k+1)^{n+1} t^{n} / n ! \leq(k+1) \cdot e^{(k+1) \cdot t} .
$$

Then

$$
E_{\Omega}[\max (\|B(\cdot, u)\|: u \leq t)] \leq E_{\Omega \times A}\left[(k+1)^{n(\cdot, \cdot, t)+1}\right] \leq(k+1) \cdot e^{(k+1) \cdot t} .
$$

(ii) Hold $t$ fixed. Let $\lambda=A \cdot \Delta t$. Then $\lambda \leq 1$. Our main difficulty is that the random variables $n(\omega, a, t), a \in A$, are not independent. The plan is to find a family of independent random variables $m(\omega, a)$ such that $n(\omega, a, t) \leq$ $m(\omega, a)$, and apply Chebyshev's inequality. We deal with the cases $\lambda \leq 1 / 2$ and $\lambda>1 / 2$ separately.

We first deal with the case $\lambda \leq 1 / 2$. The family of random variables $n(\cdot, a, t), a \in A$, may be represented in the following way on a sufficiently rich sample space. Let $\alpha=-\log (1-\lambda) / \lambda$. Then $\alpha$ increases with $\lambda$, and $\alpha \leq 2 \cdot \log 2$. Let $Y(\cdot, a, u), a \in A, u \leq t$, be a family of independent random variables each of which has value 1 with probability $\alpha \cdot \Delta t$ and value 
0 with probability $1-\alpha \cdot \Delta t$. For each $u$, the probability that there is at least one $a \in A$ such that $Y(\cdot, a, u)=1$ is

$$
1-(1-\alpha \cdot \Delta t)^{A}=1-(1-\alpha \cdot \Delta t)^{\lambda / \Delta t} \geq 1-e^{-\alpha \cdot \lambda}=\lambda .
$$

Whenever there is at least one $a \in A$ such that $Y(\omega, a, u)=1$, choose such an $a \in A$ at random using the counting measure on $A$. Let $n(\omega, a, t)$ be the number of times that $a$ is chosen for $u \leq t$. This family of random variables $n(\cdot, a, t)$ has the same joint distribution as the original family.

Now let $m(\omega, a)=\sum_{u \leq t} Y(\omega, a, u)$. The random variables $m(\cdot, a), a \in$ $A$, are independent and identically distributed, and $n(\omega, a, t) \leq m(\omega, a)$. As in the proof of Lemma 3.8, for each $a \in A$ and $n \in \mathbf{N}$,

$$
P[m(\cdot, a)=n] \leq(\alpha \cdot t)^{n} / n ! .
$$

Then as in the preceding paragraph,

$$
E\left[(k+1)^{m(\cdot, a)+1}\right] \leq(k+1) \cdot e^{\alpha \cdot(k+1) \cdot t}=M
$$

and

$$
E\left[\left((k+1)^{m(\cdot, a)+1}\right)^{2}\right] \leq L \cdot e^{\alpha \cdot L \cdot t}=S, \quad \text { where } L=(k+1)^{2} .
$$

By Chebyshev's inequality, for each $q$,

$$
P\left[\left(\sum_{a \in A}(k+1)^{m(\cdot, a)+1}\right) / A \geq M+q \cdot\left(S / A^{1 / 2}\right)\right] \leq 1 / q^{2} .
$$

Let $c=2 \cdot \log (2) \cdot(k+2)^{2}$. Taking $z \geq 1$ and $q=z \cdot A^{1 / 2}$, we have

$$
P\left[\left(\sum_{a \in A}(k+1)^{m(\cdot, a)+1}\right) / A \geq z \cdot e^{c \cdot t}\right] \leq z^{-2} \cdot A^{-1} .
$$

Since $n(\omega, a, t) \leq m(\omega, a)$, we see from the preceding paragraph that

$$
\max (\|B(\omega, u)\|: u \leq t) \leq \sum_{a \in A}\left((k+1)^{m(\omega, a)+1}\right) / A,
$$

and (ii) follows.

Now consider the case $1 / 2<\lambda \leq 1$. This time let $Y(\cdot, a, u), a \in A$, $u \leq t$, be a family of independent random variables each of which has value 1 with probability $1 / A$ and value 0 with probability $1-1 / A$. Let $r(\omega, a, t)=$ $\sum_{u \leq t} Y(\omega, a, u)$. For each $t$, the random variables $r(\omega, a, t), a \in A$, are independent. As in the first case of the proof, there is a constant $c$ depending only on $k$ such that for all $z \geq 1$,

$$
P\left[\left(\sum_{a \in A}(k+1)^{r(\cdot, a, 4 t)+1}\right) / A \geq z \cdot e^{c \cdot t}\right] \leq z^{-2} \cdot A^{-1} .
$$

Define $s(\omega, t)$ to be $\Delta t$ times the number of $u \leq t$ such that there exists $a \in A$ with $Y(\omega, a, u)=1$, and $v(\omega, t)=$ the least $u \in T$ such that $s(\omega, u)=t$. Then $v(\omega, u)<\infty$ almost surely. As in the first case of the proof, when $\lambda=1$ we may represent $n(\omega, a, t)$ by skipping times at which no agent is chosen and picking one agent from among those which are chosen otherwise. Then

$$
n(\omega, a, t) \leq r(\omega, a, v(\omega, t)) .
$$


Since $\lambda \geq 1 / 2$ and $t / \Delta t=t \cdot A / \lambda$, in general we have

$$
n(\omega, a, t) \leq r(\omega, a, v(\omega, 2 t)) .
$$

Each of the random variables $n(\cdot, a, t)$ is independent of $v(\cdot, 2 t)$. We have

$$
E[u-s(\cdot, u)]=(1-1 / A)^{A} \cdot u<u / e,
$$

so by Chebyshev's inequality,

$$
\begin{aligned}
P[v(\cdot, 2 t) \leq 4 t] & =P[4 t-s(\cdot, 4 t) \geq 2 t] \\
& \leq E[4 t-s(\cdot, 4 t)] / 2 t<2 / e .
\end{aligned}
$$

Whenever $v(\omega, 2 t) \leq 4 t$ we have $n(\omega, a, t) \leq r(\omega, a, 4 t)$ and therefore

$$
\begin{aligned}
& P\left[v(\cdot, 2 t) \leq 4 t \text { and }\left(\sum_{a \in A}(k+1)^{n(\cdot, a, t)+1}\right) / A \geq z \cdot e^{c \cdot t}\right] \\
& \quad \leq 2 e^{-1} \cdot z^{-2} \cdot A^{-1} .
\end{aligned}
$$

Since each $n(\cdot, a, t)$ is independent of $v(\cdot, 2 t)$, it follows that

$$
P\left[\left(\sum_{a \in A}(k+1)^{n \cdot(\cdot a, t)+1}\right) / A \geq z \cdot e^{c \cdot t}\right] \leq z^{-2} \cdot A^{-1},
$$

and again (ii) holds.

We shall conclude this section with results concerning relatively dense finite subsets of $\mathbf{L}^{\wedge} q$.

Lemma 3.10. For each $q$, the linear space $\left(\mathbf{L}^{\wedge} q,\||\||)\right.$ is a separable metric space, and for each constant $j>0$, the set $U_{j}$ of all $\varphi \in \mathbf{L}^{\wedge} q$ such that $\|\varphi\| \leq j$ is compact in $\left(\mathbf{L}^{\wedge} q,|||| \mid\right)$.

Proof. $(\mathbf{L} \sim q,|\||||)$ can be identified with the space of Lipschitz functions from the closed $q$-ball into $R$, and has the same topology as $\mathbf{L}^{\wedge} q$ with the sup norm. Separability follows from the Stone-Weierstrass theorem. The set $U_{j}$ is sequentially compact and therefore compact in $\left(\mathbf{L}^{\wedge} q,|||| \mid\right)$.

We will need the following sharpening of Lemma 3.10 giving a bound on the size of a finite subset of $\mathbf{L}^{\wedge} q$ which is dense up to a finite distance in the norm \|\| $\mid \|$. Let $\mathbf{L}^{\#}$ be the set of all $\varphi \in \mathbf{L}$ such that $\|\varphi\| \leq \sqrt{m}$ and $\|\varphi\| \mid \leq 1$, where $m$ is the dimension of the commodity space $C$.

Lemma 3.11. For each $q$ and each positive integer $K$, there is a finite set $H(q, K) \subset \mathbf{L}^{\#} \cap \mathbf{L}^{-} q$ such that

$$
\#(H(q, K)) \leq(2 K+1) \cdot 3^{(2 K q+1)^{m}},
$$

and for each $\varphi \in \mathbf{L}^{\wedge} q$ with $\|\varphi\| \leq 1$ there exists $\theta \in H(q, K)$ such that $\|\varphi-\theta \mid\| \leq(m+1) / K$.

Proof. Let $D$ be the set of $1 / K$-lattice points in $C$ of distance at most $q$ from the origin. Then $\#(D) \leq(2 K \cdot q+1)^{m}$. Let $I$ be the set of multiples of $1 / K$ in $[-1,1], I=\{i / K:-K \leq i \leq K, i \in Z\}$. Then $\#(I)=2 K+1$. There are at most

$$
\#(I) \cdot 3^{\#(D)}=(2 K+1) \cdot 3^{(2 q \cdot K+1)^{m}}
$$


functions $\theta: D \rightarrow I$ with Lipschitz bound 1 , because there are \#(I) possible values for $\theta(0)$ and each $\theta(x)$ has at most three possible values at the neighbors of $x$. Each such function $\theta$ can be extended to a function $\theta^{\prime}: C \rightarrow[-1,1]$ such that $\theta^{\prime} \in \mathbf{L}^{\#} \cap \mathbf{L}^{\wedge} q$ by first connecting the values along each line in the first dimension of $D$ by linear segments, then connecting the values at neighboring points in the second dimension by line segments, and so on. Let $H(q, K)$ be the set of functions $\theta^{\prime}$ formed in this way. Then $H(q, K) \subset \mathbf{L}^{\#}$ and $H(q, K)$ has cardinality at most $\#(I) \cdot 3^{\#(D)}$ as required. Consider any $\varphi \in \mathbf{L} \smile q$ with $\|\varphi\| \leq 1$. Let $\theta: D \rightarrow I$ be the function such that for each lattice point $x \in D$, $\theta(x)=[K \cdot \varphi(x)] / K$, where [.] is the greatest integer function. Let $\theta^{\prime}$ be the corresponding extension in $H(q, K)$. Then

$$
\left\|\left|\theta^{\prime}-\varphi \|\right| \leq(m+1) / K,\right.
$$

because $\varphi(x)=\varphi\left(x^{\wedge} q\right)$ and $\theta^{\prime}(x)=\theta^{\prime}\left(x^{\wedge} q\right)$, any point $x$ with $|x| \leq q$ is within $\sqrt{m} /(2 K)$ of a lattice point in $D, \theta^{\prime}$ has Lipschitz bound $\sqrt{m}, \varphi$ has Lipschitz bound $1 \leq \sqrt{m}$, and $\left|\varphi(x)-\theta^{\prime}(x)\right| \leq 1 / K$ at each lattice point $x \in D$.

Let $K$ be a positive integer, let $H(q, K)$ be the subset of $\mathbf{L}^{\#} \cap \mathbf{L}^{-} q$ from Lemma 3.11, and let $h: \mathbf{B} \rightarrow R^{H(q, K)}$ be the function defined by $(h b)(\varphi)=$ $b(\varphi), \varphi \in H(q, K)$. Give $R^{H(q, K)}$ the norm $\|y\|=\sup \{|y(\varphi)|: \varphi \in H(q, K)\}$.

Lemma 3.12. $h$ is a linear function and for all $b \in \mathbf{B}$,

$$
\|h b\| / \sqrt{m} \leq\left\|b^{\wedge} q\right\| \leq\|h b\|+\left\|b^{\wedge} q\right\| \| \cdot(m+1) / K .
$$

Proof. $h$ is obviously linear. Since $\|\varphi\| \leq \sqrt{m}$ for all $\varphi \in H(q, K)$,

$$
\begin{aligned}
\|h b\| / \sqrt{m} & =\sup \{|b(\varphi)| / \sqrt{m}: \varphi \in H(q, K)\} \\
& \leq \sup \left\{|b(\varphi)| /\|\varphi\|: 0 \neq \varphi \in \mathbf{L}^{`} q\right\} \\
& \leq \sup \left\{|b(\varphi)| /\left\|\varphi^{\wedge} q\right\|: 0 \neq \varphi \in \mathbf{L}^{\wedge} q\right\} \leq\left\|b^{\wedge} q\right\|,
\end{aligned}
$$

so $\|h b\| / \sqrt{m} \leq\left\|b^{\wedge} q\right\|$. To prove the other inequality, let $\varphi \in \mathbf{L}$ with $\|\varphi\|=1$ and let $\theta \in H(q, K)$ be such that $\left\|\theta-\varphi^{\wedge} q\right\| \| \leq(m+1) K$. Then

$$
\begin{aligned}
\left|\left(b^{\wedge} q\right)(\varphi)\right| & =\left|b\left(\varphi^{\wedge} q\right)\right| \leq|b(\theta)|+\left|b\left(\varphi^{\wedge} q-\theta\right)\right| \\
& =|h b(\theta)|+\left|b\left(\varphi^{\wedge} q-\theta\right)\right|=|h b(\theta)|+\left|\left(b^{\wedge} q\right)\left(\varphi^{\wedge} q-\theta\right)\right| \\
& \leq\|h b\|+\left|\left\|b ^ { \wedge } q \left|\left\|\cdot \left|\left\|\varphi^{\wedge} q-\theta\left|\|\leq\| h b\left\|+|| b^{\wedge} q\right\|\right| \cdot(m+1) / K,\right.\right.\right.\right.\right.\right.
\end{aligned}
$$

and therefore

$$
\left\|b^{`} q\right\| \leq\|h b\|+\left\|b^{`} q\right\| \| \cdot(m+1) / K .
$$

\section{THE HYPERFINITE PROCESS}

From this point on we shall work in an $\omega_{1}$-saturated nonstandard universe. By a starfinite exchange process we mean the transfer of the notion of a finite exchange process. We continue to write $A$ instead of $\#(A)$ for the number of elements of the set $A$. Thus $A$ is a hyperinteger.

We shall use the name hyperfinite exchange process for a starfinite exchange process which has the additional properties given by the following definition. 
Definition A. By a hyperfinite exchange process we shall mean a starfinite exchange process $(p, X)$ such that:

(a) The dimensions $d$ and $m$ are finite.

(b) The initial price $p_{0}$ and initial allocation $X_{0}(a), a \in A$, have finite uniform bound $k$.

(c) $(p, X)$ is Lipschitz with finite bound $k$.

(d) The number of agents $A$ is an infinite hyperinteger.

(e) $\delta=A^{-l}$ for some positive real number $l \in(0,1)$.

For convenience we take the same number $k$ in both conditions (b) and (c). In condition (e), $\delta$ is the price adjustment parameter of the exchange process. Recall that the definition of an exchange process includes the condition that $\Delta t \leq 1 / A$, where $\Delta t$ is the time interval length, so $\Delta t$ is infinitesimal. The sets $A$ of agents and $\Gamma$ of choices will be starfinite sets, and the price adjustment function $f$ and excess demand function $g$ will be internal functions. The sample space $\Omega$, the time line $T=\left\{n \cdot \Delta t: n \in{ }^{*} \mathbf{N}\right\}$, and the functions $F$ and $G$ corresponding to a starfinite exchange process will also be internal.

The state space $\mathbf{B}$ will be standard because it depends only on the standard dimensions $m$ and $d$. $\mathbf{B}$ is given the \|\| -metric, so that two points $b, c$ of ${ }^{*} \mathbf{B}$ are said to be infinitely close, $b \approx c$, if $\|b-c\|$ is infinitesimal, and a standard part $\operatorname{st}(b)$ of a point $b \in{ }^{*} \mathbf{B}$ is a point of $\mathbf{B}$ which is infinitely close to $b$.

Throughout this section we shall assume that $(p, X)$ is a hyperfinite exchange process.

Definition. The stochastic processes $M$ and $N$ are defined by

$$
\begin{array}{cc}
M(\omega, 0)=0, & \Delta p(\omega, t)=F(p(\omega, t), B(\omega, t)) \cdot \delta \cdot A \cdot \Delta t+\Delta M(\omega, t), \\
N(\omega, 0)=0, & \Delta B(\omega, t)=G(p(\omega, t), B(\omega, t)) \cdot \Delta t+\Delta N(\omega, t) .
\end{array}
$$

By Lemma 2.5, $M$ is a $*$-martingale with respect to $\mathbf{F}_{t}$. Similarly, by Lemma 2.9, $N$ is a $*$-martingale with respect to $\mathbf{F}_{t}$. Since $\delta \cdot A$ is infinite, we regard $p(\omega, t)$ as a fast component and $B(\omega, t)$ as a slow component in the above stochastic difference equations. The martingales $M(\omega, t)$ and $N(\omega, t)$ are the differences between the expected and actual changes in $p(\omega, t)$ and $B(\omega, t)$ at time $t$ given $\mathbf{F}_{t}$.

We now introduce some notation which we shall use throughout this section.

Definition. Let $\lambda=A \cdot \Delta t, \varepsilon=1 /(\delta \cdot A)$, and $\beta=\delta \cdot \log (1 / \varepsilon)$. Since $\Delta t \leq 1 / A$, $\lambda \leq 1 . \lambda$ is the probability that some $a \in A$ is chosen at time $t$, that is, $\omega_{A}(t+\Delta t) \in A$. By Definition $\mathrm{A}(\mathrm{e}), \delta$ and $\varepsilon$ are infinitesimal. It follows that

$$
\beta=\delta \cdot \log (A \cdot \delta) \leq A^{-l} \cdot \log (A) \approx 0,
$$

and thus $\beta$ is infinitesimal.

The next definition provides a convenient framework for keeping track of the rate at which the probability of a set approaches one.

Definition. Let $0<j \leq 1$. A set $Y \subset \Omega$ is $j$-almost sure if for each $n \in \mathbf{N}$ there is an internal set $Z \subset V$ of internal measure at least $1-j / n$.

If $j$ is standard, a set is $j$-almost sure if and only if it has Loeb measure one. The notion of $j$-almost sure becomes stronger as $j$ decreases. In this paper we 
shall come across $j$-almost sure sets where $j$ is an infinitesimal depending on $A$, such as $\left(A^{-1}\right)$-almost sure and $\exp (-A)$-almost sure. In standard terms, we shall keep track of the rate at which probabilities approach one as $A$ approaches $\infty$. This will facilitate the computation of the expected value of a hyperfinite exchange process in $\S 5$.

In this section we shall prove the following theorem.

Theorem 4.1. Let $(p, X)$ be a hyperfinite exchange process. Then the following holds $\left(A^{-n}\right)$-almost surely for each $n \in \mathbf{N}$ : For all pairs $r<s$ in $T$ such that $s$ is finite and $(s-r) / \varepsilon$ is finite,

$$
|M(\omega, s)-M(\omega, r)| \approx 0 \text { and }\|N(\omega, s)-N(\omega, r)\| \cdot \varepsilon^{-1} \approx 0 .
$$

The theorem will follow from a series of lemmas.

Lemma 4.2. Suppose $0<j \leq 1$. Then a countable intersection of $j$-almost sure sets is $j$-almost sure.

Proof. The proof is similar to the proof that Loeb measure is countably additive. Let $Y_{n}, n \in \mathbf{N}$, be a sequence of sets which are $j$-almost sure, and let $Y$ be their intersection. Let $h$ be a positive integer. For each $n$ let $Z_{n}$ be an internal subset of $Y_{n}$ of internal measure at least $1-j / 2^{h+n}$, and let $U_{n}$ be the complement of $Z_{n}$. Then for each $n \in \mathbf{N}, U_{n}$ has measure at most $j / 2^{h+n}$. By $\omega_{1}$-saturation the sequence $U_{n}, n \in \mathbf{N}$, can be extended to an internal sequence of sets $U_{n}, n \in{ }^{*} \mathbf{N}$, such that each $U^{n}$ is a subset of $\Omega$ of measure at most $j / 2^{h+n}$. Let $K$ be an infinite hyperinteger and let $U$ be the union of $U_{n}, n \leq K$. Then $U$ is an internal set of measure at most $j / 2^{h-1}$. The complement of $U$ is an internal subset of $Y$ of measure at least $1-j / 2^{h-1}$, as required.

We shall first consider processes in which the commodity holding $X(\omega, a, t)$ is uniformly bounded, and then deal with the general case.

Definition. The hyperfinite exchange process $(p, X)$ is said to be uniformly bounded by a hyperreal number $Q$ if $|X(\omega, a, t)| \leq Q$ for all $\omega, a$, and $t$. The truncation of $(p, X)$ at $Q$ is the process obtained by replacing the excess demand function $g$ by the new function

$$
g^{\wedge}(\gamma, p, x)=[x+g(\gamma, p, x)]^{\wedge} Q-x
$$

and replacing the initial allocation $X(a, 0)$ by $X(a, 0)^{\wedge} Q$.

The hyperreal number $Q$ may be finite or infinite. Note that if $(p, X)$ is uniformly bounded by $Q$ then $\|B(\omega, t)\| \leq Q+1$ for all $\omega$ and $t$, because

$$
|||B(\omega, t)| \| \mid=B(\omega, t)(\mathrm{abs})=E_{A}[|X(\omega, \cdot, t)|+1] \leq Q+1 .
$$

For any hyperfinite exchange process $(p, X)$, the truncation $\left(p^{\wedge}, X^{\wedge}\right)$ of $(p, X)$ at $Q$ is again a hyperfinite exchange process. $\left(p^{\wedge}, X^{\wedge}\right)$ is uniformly bounded by $Q$, because we always have $\left|x+g^{\wedge}(\gamma, p, x)\right| \leq Q$. Moreover, if $\omega, t$ are such that $|X(\omega, a, s)| \leq Q$ for all $a \in A$ and all $s \leq t$, then $X(\omega, a, s)=X^{\wedge}(\omega, a, s)$ and $p(\omega, s)=p^{\wedge}(\omega, s)$ for all $a \in \bar{A}$ and all $s \leq t$.

For $i \leq d$, let $M_{i}$ be the $i$ th coordinate of $M$. 
Lemma 4.3. Suppose that $(p, X)$ is uniformly bounded by $Q$. Then for each $s \in T$ and $\omega \in \Omega$ and each coordinate $i \leq d$,

(i) $|\Delta M(\omega, s)| \leq k \cdot(Q+1) \cdot \delta \cdot(1+\lambda)$.

(ii) $E_{\Omega}\left[\left(\Delta M_{i}(\cdot, s)\right)^{2} \mid \mathbf{F}_{t}\right](\omega) \leq[k \cdot(Q+1) \cdot \delta]^{2} \cdot \lambda$.

Proof. (i) If an agent $a \in A$ is chosen at time $t$, then

$$
\begin{aligned}
|\Delta p(\omega, t)| & =|f(\omega, p(\omega, t), X(\omega, a, t)) \cdot \delta| \\
& \leq k \cdot(|X(\omega, a, t)|+1) \cdot \delta \leq k \cdot(Q+1) \cdot \delta .
\end{aligned}
$$

Otherwise $\Delta p(\omega, t)=0$. In either case

$$
\begin{aligned}
|\Delta M(\omega, t)| & \leq|\Delta p(\omega, t)|+|F(p(\omega, t), B(\omega, t)) \cdot \delta \cdot \lambda| \\
& \leq k \cdot(Q+1) \cdot \delta+k \cdot(Q+1) \cdot \delta \cdot \lambda=k \cdot(Q+1) \cdot \delta \cdot(1+\lambda),
\end{aligned}
$$

by Lemma 2.6. Part (ii) is shown by the following computation:

$$
\begin{aligned}
E_{\Omega}[ & \left.\left(\Delta M_{i}(\cdot, t)\right)^{2} \mid \mathbf{F}_{t}\right](\omega) \\
\quad & =E_{\Omega}\left[\left(\Delta p_{i}(\cdot, t)\right)^{2} \mid \mathbf{F}_{t}\right](\omega)-F_{i}(p(\omega, t), B(\omega, t))^{2} \cdot(\delta \cdot \lambda)^{2} \\
& \leq E_{\Omega}\left[\left(\Delta p_{i}(\cdot, t)\right)^{2} \mid \mathbf{F}_{t}\right](\omega) \leq[k \cdot(Q+1) \cdot \delta]^{2} \cdot \lambda .
\end{aligned}
$$

Definition. Let $U$ be the set of all pairs $(r, s) \in T \times T$ such that $r \leq s, s$ is finite, and $s-r \leq \varepsilon$.

Lemma 4.4. Let $v$ be a real number between 0 and $1 / 2$ and let $Q \geq 1$ be hyperreal. Let $(p, X)$ be uniformly bounded by $Q$. Then there is a real number $j$ (depending only on $l$ and $v$ ) such that the following holds $\exp \left(-A^{j}\right)$-almost surely: For all $(r, s) \in U$,

$$
|M(\omega, s)-M(\omega, r)| \leq Q \cdot \beta^{v} .
$$

Proof. Let $\alpha \in(0,1), \alpha^{\prime}=\log (2-\alpha), y=2 k \cdot(Q+1) \cdot \delta$, and $\sigma=k \cdot(Q+$ 1) $\cdot \delta \cdot \sqrt{A}$. By Lemma $4.3,|\Delta M(\omega, s)| \leq y$ and

$$
E_{\Omega}\left[\left|\left(\Delta M_{i}(\cdot, s)\right)^{2}\right| \mathbf{F}_{s}\right](\omega) \leq[k \cdot(Q+1) \cdot \delta]^{2} \cdot \lambda=\sigma^{2} \cdot \Delta t
$$

for all $s \in T$ and $\omega \in \Omega$. Let $r \in T$ and let $s=r+\varepsilon$. By Bernstein's Inequality, whenever $0<z<\sigma \cdot \alpha^{\prime} \cdot y^{-1} \cdot \sqrt{\varepsilon}=\alpha^{\prime} / 2 \sqrt{\delta}$, we have

$$
P_{\Omega}\left[\max _{r \leq u \leq s}|M(\omega, u)-M(\omega, r)| \geq z \cdot \sigma \cdot \sqrt{\varepsilon}\right] \leq 2 e^{-\alpha z^{2} / 2} .
$$

Let $\gamma$ be infinitesimal and $\gamma \geq \beta$, and let

$$
z^{2}=(4 \gamma) /(\delta \cdot \alpha)=4 \log \left(\varepsilon^{-1}\right) \cdot \gamma /(\beta \cdot \alpha) .
$$

Then

$$
z=2 \sqrt{\gamma} \cdot(\delta \cdot \alpha)^{-1 / 2} .
$$

Since $\gamma \approx 0, z$ is small enough so that Bernstein's Inequality above is applicable whenever $s-r \geq \varepsilon$. Moreover, from the definition of $z$ we see that $z$ is positive infinite. Since $z$ is positive infinite and $\alpha$ is positive real,

$$
\begin{gathered}
\log \left[\varepsilon^{-1} \cdot \exp \left(-\alpha \cdot z^{2} / 2\right)\right]=\log \left(\varepsilon^{-1}\right)-\alpha \cdot z^{2} / 2 \\
=\log \left(\varepsilon^{-1}\right) \cdot(1-2 \gamma / \beta) \leq \log \left(\varepsilon^{\gamma / \beta}\right) .
\end{gathered}
$$


For each positive integer $n$, any pair $(r, u)$ such that $0 \leq r \leq u \leq n$ and $u-r \leq \varepsilon / 2$ is covered by one of the $2 n / \varepsilon$ intervals $[K \cdot \varepsilon / 2, K \cdot \varepsilon / 2+\varepsilon]$, where $K$ is a hyperinteger below $n / \varepsilon$. It follows from Bernstein's inequality that the probability that there exists a pair $(r, s)$ in $T$ with $r \leq s \leq n$ and $s-r \leq \varepsilon$ such that

$$
|M(\omega, s)-M(\omega, r)| \geq 2 z \cdot \sigma \cdot \sqrt{\varepsilon}=4 k \cdot(Q+1) \cdot \sqrt{\gamma} / \sqrt{\alpha}
$$

is at most

$$
4 n \cdot \varepsilon^{-1} \cdot \exp \left(-\alpha \cdot z^{2} / 2\right) \leq 4 n \cdot \varepsilon^{\gamma / \beta} .
$$

Now take $\gamma=\beta^{w}$, where $2 v<w<1$. Then any finite multiple of $\sqrt{\gamma}$ is less than $\beta^{v}$, but

$$
\begin{aligned}
4 n \cdot \varepsilon^{\gamma / \beta} & \leq \exp (-\gamma / \beta)=\exp \left(-\beta^{w-1}\right)=\exp \left(-(\delta \cdot \log (1 / \varepsilon))^{w-1}\right) \\
& \leq \exp \left(-A^{-(c / 2) \cdot(w-1)}\right)=\exp \left(-A^{h}\right),
\end{aligned}
$$

where $h=-l \cdot(w-1) / 2$ is a positive real number. Thus, for any $j<h$, the inequality in the lemma holds up to time $n \exp \left(-A^{j}\right)$-almost surely. By Lemma 4.2, the inequality holds for all finite times $\exp \left(-A^{j}\right)$-almost surely.

We remark that if we weaken the hypothesis $\delta=A^{-l}$ to $\delta \approx \varepsilon \approx \beta \approx 0$, then $4 n \cdot \varepsilon^{\gamma / \beta}$ is still infinitesimal, and therefore Lemma 4.4 still holds $\exp \left(-\beta^{-j}\right)$ almost surely for some positive real $j$.

The next lemma gives bounds for $\Delta N(\omega, t)(\varphi)$ for a single function $\varphi \in \mathbf{L}$.

Lemma 4.5. Suppose that $(p, X)$ is uniformly bounded by $Q$. Let $\varphi \in \mathbf{L}$, $t \in T$, and $\omega \in \Omega$. Then

(i) $|\Delta N(\omega, t)(\varphi)| \leq(k+2) \cdot(Q+1) \cdot \varepsilon \cdot \delta \cdot(1+\lambda) \cdot\|\varphi \mid\|$.

(ii) $E_{\Omega}\left[(\Delta N(\cdot, t)(\varphi))^{2} \mid \mathbf{F}_{t}\right](\omega) \leq \lambda \cdot\left((k+2)(Q+1) \cdot \varepsilon \cdot \delta \cdot|\|\varphi \mid\|)^{2}\right.$.

Proof. (i) If an agent $a \in A$ is chosen at time $t$, then putting $\left.\gamma=\omega_{\Gamma}(t+\Delta t)\right)$,

$$
\begin{aligned}
\mid \Delta B & (\omega, t)(\varphi)|=| \varphi(X(\omega, a, t+\Delta t))-\varphi(X(\omega, a, t)) \mid / A \\
& =|\varphi(X(\omega, a, t)+g(\gamma, p(\omega, t), X(\omega, a, t)))-\varphi(X(\omega, a, t))| / A \\
& \leq[|\varphi(X(\omega, a, t)+g(\gamma, p(\omega, t), X(\omega, a, t)))|+|\varphi(X(\omega, a, t))|] / A \\
& \leq(k+2) \cdot(Q+1) \cdot|||\varphi||| / A .
\end{aligned}
$$

If no agent is chosen at time $t$, then $\Delta B(\omega, t)=0$. The probability that some agent is chosen at time $t$ is $\lambda$, so by Lemma 2.9

$$
\begin{aligned}
& |G(p(\omega, t), B(\omega, t))(\varphi) \cdot \Delta t|=\left|E_{\Omega}\left[\Delta B(\cdot, t)(\varphi) \mid \mathbf{F}^{t}\right](\omega)\right| \\
& \quad \leq(k+2) \cdot(Q+1) \cdot|||\varphi|\|\cdot \lambda / A=(k+2) \cdot(Q+1) \cdot\||||| \mid \cdot \Delta t .
\end{aligned}
$$

Suppose some agent is chosen at time $t$. Then

$$
\begin{aligned}
|\Delta N(\omega, t)(\varphi)| & \leq|\Delta B(\omega, t)(\varphi)|+|G(p(\omega, t), B(\omega, t))(\varphi) \cdot \Delta t| \\
& \leq(k+2) \cdot(Q+1) \cdot|||\varphi||| \cdot(1 / A+\Delta t) \\
& =(k+2) \cdot(Q+1) \cdot \varepsilon \cdot \delta \cdot(1+\lambda) \cdot|||\varphi||| .
\end{aligned}
$$

Now suppose no agent is chosen at time $t$. Then

$$
\begin{aligned}
|\Delta N(\omega, t)(\varphi)| & =|G(p(\omega, t), B(\omega, t))(\varphi) \cdot \Delta t| \\
& \leq(k+2) \cdot(Q+1) \cdot|\| \varphi||| \cdot \Delta t \\
& =(k+2) \cdot(Q+1) \cdot \varepsilon \cdot \delta \cdot \lambda \cdot|\|\varphi \mid\| .
\end{aligned}
$$

In each case, (i) holds. 
(ii)

$$
\begin{aligned}
E_{\Omega}[ & \left.(\Delta N(\cdot, t)(\varphi))^{2} \mid \mathbf{F}_{t}\right](\omega) \\
& =E_{\Omega}\left[(\Delta B(\cdot, t)(\varphi))^{2} \mid \mathbf{F}_{t}\right](\omega)-[G(p(\omega, t), B(\omega, t))(\varphi) \cdot \Delta t]^{2} \\
& \leq[(k+2) \cdot(Q+1) \cdot\|\| \varphi \mid \| / A]^{2} \cdot \lambda \\
& =[(k+2) \cdot(Q+1) \cdot\|\varphi \mid\| \cdot \varepsilon \cdot \delta]^{2} \cdot \lambda .
\end{aligned}
$$

The next lemma shows that if the hyperfinite exchange process is uniformly bounded by $Q$ and $s$ is finite and within $\varepsilon$ of $r$, then $\|N(\omega, s)-N(\omega, r)\|$ has order $Q \cdot \varepsilon$. Later on we shall show that when $s$ is finite and within $\varepsilon$ of $r,\|N(\omega, s)-N(\omega, r)\|$ is infinitesimal compared to $\varepsilon$.

Lemma 4.6. There is a positive real number $c_{0}$ which depends only on $k$ such that if $(p, X)$ is uniformly bounded by $Q$ and $Q \geq 1$ then the following holds $\exp \left(-A^{j}\right)$-almost surely for every real $j<l$ : For all $(r, s) \in U,\|\| N(\omega, s)-$ $N(\omega, r) \mid \| \leq c_{0} \cdot Q \cdot \varepsilon$.

Proof. By the proof of Lemma 4.5,

$$
\|\Delta N(\omega, s)\| \mid \leq(k+2) \cdot(Q+1) \cdot \varepsilon \cdot \delta \cdot(1+\lambda)
$$

if some agent $a \in A$ is chosen at time $s$, which happens with probability $\lambda$, and

$$
\|\Delta N(\omega, s)\| \mid \leq(k+2) \cdot(Q+1) \cdot \varepsilon \cdot \delta \cdot \lambda
$$

otherwise.

Fix an element $u \in T$. Let $Y(\omega, s), s \geq u$, be the number of $r \in T$ such that $u \leq r<s$ and some agent $a \in A$ is chosen at time $r$. The process $Y(\omega, s)$ has independent increments and $E_{\Omega}\left[\Delta Y(\cdot, s) \mid \mathbf{F}_{s}\right]=\lambda$. For each $s \geq u$ we have

$$
\begin{aligned}
& \|N(\omega, s)-N(\omega, u)\| \\
& \quad \leq(k+2) \cdot(Q+1) \cdot \varepsilon \cdot \delta \cdot[(1+\lambda) \cdot Y(\omega, s)+\lambda \cdot((s-u) / \Delta t-Y(\omega, s))] \\
& \quad=(k+2) \cdot(Q+1) \cdot \varepsilon \cdot \delta \cdot[Y(\omega, s)+A \cdot(s-u)] .
\end{aligned}
$$

Let $Z(\omega, s), u \leq s$, be the $\mathbf{F}_{s}$-martingale $Z(\omega, s)=Y(\omega, s)-A \cdot(s-u)$. Then for each $s$ we have $|\Delta Z(\omega, s)| \leq 1$ and

$$
\begin{aligned}
E_{\Omega}\left[(\Delta Z(\omega, s))^{2} \mid \mathbf{F}_{s}\right] & \leq \lambda^{2} \cdot(1-\lambda)+(1-\lambda)^{2} \cdot \lambda \\
& =\lambda \cdot(1-\lambda) \leq \lambda .
\end{aligned}
$$

Let $\alpha \in(0,1)$ and $\alpha^{\prime}=\log (2-\alpha)$. By Bernstein's inequality, if $\lambda \leq \sigma^{2} \cdot \Delta t$ and $0<z \leq \alpha^{\prime} \cdot \sigma \cdot \sqrt{\varepsilon}$, then

$$
P_{\Omega}\left[\max _{u \leq s \leq u+\varepsilon}|Z(\omega, s)| \geq z \cdot \sigma \cdot \sqrt{\varepsilon}\right] \leq 2 e^{-\alpha z^{2} / 2} .
$$

Thus, putting $\sigma^{2}=A$ and $z=\alpha^{\prime} \cdot \sigma \cdot \sqrt{\varepsilon}=\alpha^{\prime} / \sqrt{\delta}$, we have $\lambda=\sigma^{2} \cdot \Delta t$ and

$$
P_{\Omega}\left[\max _{u \leq s \leq u+\varepsilon}|Z(\omega, s)| \geq \alpha^{\prime} / \delta\right] \leq 2 e^{-\alpha^{\cdot} \alpha^{\prime 2} /(2 \delta)} .
$$

Whenever $u \leq s \leq u+\varepsilon$ and $|Z(\omega, s)| \leq \alpha^{\prime} / \delta$ we have

$$
Y(\omega, s) \leq \alpha^{\prime} / \delta+A \cdot \varepsilon=\left(1+\alpha^{\prime}\right) / \delta
$$


and therefore

$$
\begin{aligned}
\|N(\omega, s)-N(\omega, u)\| & \leq(k+2) \cdot(Q+1) \cdot \varepsilon \cdot \delta \cdot[Y(\omega, s)+A \cdot \varepsilon] \\
& \leq(k+2) \cdot(Q+1) \cdot \varepsilon \cdot \delta \cdot\left[\left(1+\alpha^{\prime}\right) / \delta+1 / \delta\right] \\
& =(k+2) \cdot(Q+1) \cdot \varepsilon \cdot\left(2+\alpha^{\prime}\right) .
\end{aligned}
$$

Since $\delta=A^{-l}$ and $\varepsilon=A^{l-1}$, we have

$$
\varepsilon^{-1} \cdot e^{-\alpha \cdot \alpha^{\prime 2} /(2 \delta)} \leq e^{-A^{j}}
$$

for any positive real number $j<l$. Let $t \in T$ be finite. There are $2 t / \varepsilon$ subintervals of $[0, t]$-length $\varepsilon$ starting from a multiple of $\varepsilon / 2$, and any pair $u \leq s \leq t$ with $t-u \leq \varepsilon / 2$ is contained on one of these intervals. Therefore, for every real $j<l$, the following holds $\exp \left(-A^{j}\right)$-almost surely: Whenever $u \leq s \leq t$ with $s-u \leq \varepsilon$, we have

$$
\|N(\omega, s)-N(\omega, u)\| \mid \leq 4(k+2) \cdot(Q+1) \cdot \varepsilon \cdot\left(2+\alpha^{\prime}\right) .
$$

Thus, by Lemma 4.2 , the result holds with $c_{0}=8(k+2) \cdot\left(2+\alpha^{\prime}\right)$.

We remark that as in the case of Lemma 4.4, if we weaken the hypothesis $\delta=A^{-l}$ to $\delta \approx \varepsilon \approx \beta \approx 0$, then Lemma 4.6 still holds $\exp \left(-\beta^{-j}\right)$-almost surely for some positive real $j$.

Lemma 4.7. Suppose that $(p, X)$ is uniformly bounded by $Q$. Let $H$ be a *-finite subset of $\mathbf{L}$ such that $\|\varphi\| \| \leq 1$ for all $\varphi \in H$. Suppose that $r<s$ in $T$ and $0<z \leq 0.2 \cdot[(s-r) \cdot A]^{1 / 2}$. Then the probability that there exists $\varphi \in H$ and $u \in T$ such that $r \leq u \leq s$ and

$$
|(N(\omega, u)-N(\omega, r))(\varphi)| \geq z \cdot(k+2) \cdot(Q+1) \cdot[(s-r) / A]^{1 / 2}
$$

is at most $2 \cdot \#(H) \cdot \exp \left(-z^{2} / 4\right)$.

Proof. Let $\varphi \in H$. By Lemma 4.5, for all $\omega \in \Omega$ and $s \in T$,

$$
|\Delta N(\omega, s)(\varphi)| \leq(k+2) \cdot(Q+1) \cdot \varepsilon \cdot \delta \cdot(1+\lambda) \leq 2(k+2) \cdot(Q+1) / A
$$

and

$$
\begin{aligned}
& E_{\Omega}\left[(\Delta N(\cdot, s))^{2} \mid \mathbf{F}_{s}\right](\omega) \\
& \quad \leq \lambda \cdot[(k+2) \cdot(Q+1) \cdot \varepsilon \cdot \delta]^{2}=[(k+2) \cdot(Q+1)]^{2} \cdot \Delta t / A .
\end{aligned}
$$

Take $\alpha=1 / 2, \alpha^{\prime}=\log (2-\alpha)>0.4$, and $\sigma=(k+2) \cdot(Q+1) / \sqrt{A}$. By Bernstein's inequality, we have

$$
P_{\Omega}\left[\max _{r \leq u \leq s}|N(\omega, u)(\varphi)-N(\omega, r)(\varphi)| \geq z \cdot \sigma \cdot \sqrt{s-r}\right] \leq 2 e^{-\alpha z^{2} / 2} .
$$

The result now follows by substituting the values of $\alpha$ and $\sigma$ in the above inequality, and applying the inequality once for each $\varphi \in H$.

Lemma 4.8. Suppose that $(p, X)$ is uniformly bounded by $Q$. Let $q=(Q+$ $1) \cdot(k+1)$. Then for all $\omega$ and $t$,

(i) $B(\omega, t)=B(\omega, t) \wedge Q$.

(ii) $G(p(\omega, t), B(\omega, t))=G(p(\omega, t), B(\omega, t))^{\wedge} q$.

(iii) $N(\omega, t)=N(\omega, t) \wedge q$. 
Proof. For brevity we shall write $p=p(\omega, t), X(a)=X(\omega, a, t)$, and $B=$ $B(\omega, t)$.

(i) For each $a \in A$ and $\varphi \in \mathbf{L}$ we have

$$
X(a)=X(a)^{\wedge} Q, \quad \varphi(X(a))=\varphi\left(X(a)^{\wedge} Q\right)=\left(\varphi^{\wedge} Q\right)(X(a)),
$$

and therefore

$$
B(\varphi)=E_{A}[\varphi(X(\cdot))]=E_{A}\left[\left(\varphi^{\wedge} Q\right)(X(\cdot))\right]=B\left(\varphi^{\wedge} Q\right)=\left(B^{\wedge} Q\right)(\varphi) .
$$

(ii) For each $a \in A$ and $\gamma \in \Gamma$,

$$
\begin{aligned}
& |g(\gamma, p, X(a))| \leq k \cdot(|X(a)|+1) \leq k \cdot(Q+1), \\
& |X(a)+g(\gamma, p, X(a))| \leq(k+1) \cdot(Q+1)=q .
\end{aligned}
$$

Then, for each $\varphi \in \mathbf{L}$,

$$
\varphi(X(a)+g(\gamma, p, X(a)))=\left(\varphi^{\wedge} q\right)(X(a)+g(\gamma, p, X(a)))
$$

and

$$
\varphi(X(a))=\left(\varphi^{\wedge} q\right)(X(a)) .
$$

Then

$$
\begin{aligned}
\bar{g}(p, \varphi)(X(a)) & =E_{\Gamma}[\varphi(X(a)+g(\cdot, p, X(a)))-\varphi(X(a))] \\
& =E_{\Gamma}\left[\left(\varphi^{\wedge} q\right)(X(a)+g(\cdot, p, X(a)))-\left(\varphi^{\wedge} q\right)(X(a))\right] \\
& =\bar{g}\left(p, \varphi^{\wedge} q\right)(X(a)) .
\end{aligned}
$$

It follows that for each $\varphi$,

$$
\begin{aligned}
G(p, B)(\varphi) & =B(\bar{g}(p, \varphi))=B\left(\bar{g}\left(p, \varphi^{\wedge} q\right)\right) \\
& =G(p, B)(\varphi \prec q)=(G(p, B) \prec q)(\varphi) .
\end{aligned}
$$

Since the mapping $b \rightarrow b^{\wedge} q$ is linear, (iii) follows from (i) and (ii) and the equation

$$
N(\omega, t)=B(\omega, t+\Delta t)-B(\omega, t)-G(p, B(\omega, t)) \cdot \Delta t .
$$

The next lemma gives a bound for $\|N(\omega, s)-N(\omega, r)\|$. Using the bounds for $\Delta N(\omega, t)(\varphi)$ in Lemma 4.5, we could get a bound for $\mid N(\omega, s)(\varphi)-$ $N(\omega, r)(\varphi) \mid$ for each function $\varphi \in \mathbf{L}$ by using Bernstein's inequality. However, since the space $(\mathbf{B},\|\|)$ is not separable, we cannot go directly from a bound for single functions $\varphi$ to a bound for the norm $\|N(\omega, s)-N(\omega, r)\|$. Instead we use Lemma 4.7 and the approximation lemmas from $\S 3$. As before, let $U$ be the set of all $(r, s) \in T \times T$ such that $r<s, s$ is finite, and $(s-r) \leq \varepsilon$. Recall that $m$ is the dimension of the commodity space $C$.

Lemma 4.9. Let $v, w$, and $j$ be positive real numbers such that $v+w<$ $1 /(m+2)$ and $j<l \cdot m /(m+2)$. If $(p, X)$ is uniformly bounded by $Q$ and $1 \leq Q \leq \beta^{-v}$, then the following holds $\exp \left(-A^{j}\right)$-almost surely: For each $(r, s) \in U,\|N(\omega, s)-N(\omega, r)\| \leq \varepsilon \cdot Q \cdot \beta^{w}$.

Proof. By Lemma 4.6, there is a positive real $c_{1}$ such that for real $j<l$, the following holds $\exp \left(-A^{j}\right)$-almost surely: For all $(r, s) \in U$,

$$
\|N(\omega, s)-N(\omega, r)\| \mid \leq c_{1} \cdot(Q+1) \cdot \varepsilon .
$$


Let $q=(k+2) \cdot(Q+1), z=\beta^{1 /(m+2)} / \sqrt{\delta}$, and $K=\beta^{v-1 /(m+2)}$. Since $\beta$ is infinitesimal, $K$ is infinite. Let $H(q, K)$ be the subset of $\mathbf{L}^{\#} \cap \mathbf{L}^{\sim} q$ from Lemma 3.11. Then

$$
\#(H(q, K)) \leq(2 K+1) \cdot 3^{(2 K \cdot q+1)^{m}} .
$$

We wish to apply Lemma 4.7. Suppose $s-r=2 \varepsilon$. Since $\beta \approx 0$ we have

$$
0<z<0.2 \cdot \sqrt{2} / \sqrt{\delta}=0.2 \cdot[2 \varepsilon \cdot A]^{1 / 2}=0.2 \cdot[(s-r) \cdot A]^{1 / 2}
$$

as required in the hypothesis of Lemma 4.7. By Lemma 4.7, the probability that

$$
\begin{aligned}
|(N(\omega, u)-N(\omega, r))(\varphi)| & \leq z \cdot q \cdot[(s-r) / A]^{1 / 2} \\
& =\sqrt{2} \cdot q \cdot \varepsilon \cdot \beta^{1 /(m+2)}
\end{aligned}
$$

for all $r \leq u \leq s$ and all $\varphi \in H(q, K)$ is at least

$$
1-2 \cdot \#(H(q, K)) \cdot \exp \left(-z^{2} / 4\right) \text {. }
$$

Let $n$ be a positive integer. Then the probability that

$$
|(N(\omega, s)-N(\omega, r))(\varphi)| \leq 2 \sqrt{2} \cdot q \cdot \varepsilon \cdot \beta^{1 /(m+2)}
$$

for all $r<s \leq n$ in $T$ with $s-r \leq \varepsilon$ and all $\varphi \in H(q, K)$ is at least

$$
1-2 n \cdot \varepsilon^{-1} \cdot \#(H(q, K)) \cdot \exp \left(-z^{2} / 4\right) \text {. }
$$

Let $h: \mathbf{B} \rightarrow R^{H(q, K)}$ be the linear function introduced in Lemma 3.12. Since the norm in the Euclidean space $R^{H(q, K)}$ is the maximum of the absolute values of the coordinates, the probability that

$$
\|h(N(\omega, s)-N(\omega, r))\| \leq 2 \sqrt{2} \cdot q \cdot \varepsilon \cdot \beta^{1 /(m+2)}
$$

for all $r<s \leq n$ in $T$ with $s-r \leq \varepsilon$ is at least the value of (2).

We now estimate the probability (2). We show that the logarithm of $\varepsilon^{-1}$. $\#(H(q, K)) \cdot \exp \left(-z^{2} / 4\right)$ is less than $-A^{j}$. Put $L=\log \left(\varepsilon^{-1}\right)$. We have

$$
\begin{aligned}
\log \left(\varepsilon^{-1} \cdot \#(H(q, K)) \cdot \exp \left(-z^{2} / 4\right)\right) \\
\quad=L-z^{2} / 4+\log (\#(H(q, K))) \\
\quad \leq L-z^{2} / 4+3^{m} \cdot K^{m} \cdot q^{m} \cdot \log (3) \\
\quad=L-\beta^{2 /(m+2)} / 4 \delta+c_{2} \cdot \beta^{m \cdot(v-1 /(m+2))} \cdot q^{m} \\
\quad \leq L-\beta^{2 /(m+2)} / 4 \delta+c_{3} \cdot \beta^{m \cdot(v-1 /(m+2))} \cdot \beta^{-m \cdot v} \\
\quad=L \cdot \beta^{2 /(m+2)} \cdot L / 4 \beta+c_{3} \cdot \beta^{-m /(m+2)} \\
\quad=L-\beta^{-m /(m+2)} \cdot L / 4+c_{3} \cdot \beta^{-m /(m+2)} \\
\quad<-\beta^{-m /(m+2)}=-\left(A^{-l} \cdot \log \left(A^{1-l}\right)\right)^{-m /(m+2)}<-A^{j},
\end{aligned}
$$

where $c_{2}=3^{m} \cdot \log (3)$ and $c_{3}=c_{2} \cdot 2^{m} \cdot(k+2)^{m}$, because $L$ is infinite and $c_{3}$ is finite. Hence the probability (2) is at least $1-\exp \left(-A^{j}\right)$. Since this holds for all positive integers $n$, it follows (using Lemma 4.2) that $\exp \left(-A^{j}\right)$-almost surely, for all $(r, s) \in U$, we have

$$
\|h(N(\omega, s)-N(\omega, r))\| \leq 2 \sqrt{2} \cdot q \cdot \varepsilon \cdot \beta^{1 /(m+2)} .
$$


By Lemma 4.8, $N(\omega, t)=N(\omega, t)^{\wedge} q$ for all $\omega, t$. Then by (1), (3), and Lemma 3.12, the following holds $\exp \left(-A^{j}\right)$-almost surely: For all $(r, s) \in U$ we have

$$
\begin{aligned}
& \|N(\omega, s)-N(\omega, r)\|=\left\|N(\omega, s)^{\wedge} q-N(\omega, r)^{\wedge} q\right\| \\
& \quad \leq 2 \sqrt{2} \cdot q \cdot \varepsilon \cdot \beta^{1 /(m+2)}+\left\|N(\omega, s)^{\wedge} q-N(\omega, r)^{\wedge} q\right\| \cdot(m+1) / K \\
& \quad \leq 2 \sqrt{2} \cdot q \cdot \varepsilon \cdot \beta^{1 /(m+2)}+c_{1} \cdot(Q+1) \cdot \varepsilon \cdot(m+1) / K \\
& \quad \leq \varepsilon \cdot Q \cdot c_{4} \cdot \beta^{1 /(m+2)-v} \leq \varepsilon \cdot Q \cdot \beta^{w} .
\end{aligned}
$$

This completes the proof.

At this point we can prove the analogue of Theorem 4.1 with the additional hypothesis that the hyperfinite exchange process $(p, X)$ has a finite uniform bound but with the hypothesis $\delta=A^{-l}$ weakened to $\delta \approx \varepsilon \approx \beta \approx 0$. As with earlier lemmas, under these hypotheses Lemma 4.9 holds $\exp \left(-\beta^{-j}\right)$-almost surely for some positive real $j$.

Theorem 4.10. Let $(p, X)$ be a hyperfinite exchange process but with Definition $\mathrm{A}(\mathrm{e})$ replaced by the condition that $(p, X)$ has a finite uniform bound and that $\delta, \varepsilon$, and $\beta$ are infinitesimal. Then there is a positive real $j$ such that the following holds $\exp \left(-\beta^{-j}\right)$-almost surely: For all pairs $r<s$ in $T$ such that $s$ is finite and $(s-r) / \varepsilon$ is finite,

$$
|M(\omega, s)-M(\omega, r)| \approx 0 \text { and }\|N(\omega, s)-N(\omega, r)\| \cdot \varepsilon^{-1} \approx 0 .
$$

Proof. Under the assumption $\beta \approx 0$, Lemmas 4.4 and 4.9 hold $\exp \left(-\beta^{-j}\right)$ almost surely for positive real $j$. By the triangle inequality, it suffices to prove that $\exp \left(-\beta^{-j}\right)$-almost surely, for all pairs $(r, s) \in U$,

$$
|M(\omega, r)-M(\omega, s)| \approx 0 \text { and }\|N(\omega, s)-N(\omega, r)\| / \varepsilon \approx 0 .
$$

By Lemma 4.4 with a finite uniform bound $Q$,

$$
|M(\omega, r)-M(\omega, s)| \approx 0
$$

for all pairs $(r, s) \in U \exp \left(-\beta^{-j}\right)$-almost surely for some positive real $j$. Let $w$ be a real number between 0 and $1 /(m+2)$. By Lemma 4.9, there is a positive real $j$ such that $\exp \left(-\beta^{-j}\right)$-almost surely, for all $(r, s) \in U$,

$$
\|N(\omega, s)-N(\omega, r)\| \leq \varepsilon \cdot Q \cdot \beta^{w},
$$

and hence $\|N(\omega, s)-N(\omega, r)\| / \varepsilon \approx 0$.

We now deal with the unbounded case. Our next lemma shows that we can find a hyperreal number $Q$ which is not too large such that $\left(A^{-j}\right)$-almost surely, the given process will be the same as the process truncated at $Q$ for all finite times.

Lemma 4.11. Let $Q=A^{q}$, where $q$ is a positive real number. Then for each finite $j$, the following holds $\left(A^{-j}\right)$-almost surely:

$$
|X(\omega, a, s)| \leq Q \text { for all } a \in A \text { and all finite } s \in T .
$$

Proof. Take a positive real number $j$. Let $t \in T$ and let $n(\omega, a, t)$ be the number of times that trader $a$ is chosen during the time interval $0 \leq u \leq t$. By Lemma 3.8(i), whenever $n \geq 2 t$ we have

$$
P_{\Omega}[n(\cdot, a, t) \geq n] \leq \sum_{i=n}^{\infty} t^{i} / i ! \leq 2 \cdot t^{n} / n ! .
$$


Now let $n$ be the least hyperinteger such that $(k+1)^{n+1} \geq Q+1$. Then the probability that $(k+1)^{n(\cdot, a, t)+1} \geq Q+1$ for some $a \in A$ is at most $A \cdot 2 t^{n} / n !$. $n$ is infinite, so $t^{n} / n$ ! is infinitesimal for all finite $t$. However, the finite number $s=(k+1)^{2 / q}$ has the property that

$$
s^{n}=(k+1)^{2 n / q}>(k+1)^{(n+1) / q}>Q^{1 / q}=A .
$$

It follows that $A \cdot 2 t^{n} / n !<A^{-j}$ for all finite $t$. Therefore, the following holds $\left(A^{-j}\right)$-almost surely:

$$
(k+1)^{n(\cdot, a, t)+1}<Q+1 \text { for all } a \in A .
$$

By Lemma 3.8(ii),

$$
\operatorname{abs}(X(\omega, a, u)) \leq(k+1)^{n(\omega, a, u)+1} \leq(k+1)^{n(\omega, a, t)+1}
$$

for all $u \leq t$. Therefore for each finite $t \in T$,

$$
|X(\omega, a, s)|<Q \text { for all } a \in A \text { and all } s \leq t,
$$

$\left(A^{-j}\right)$-almost surely. By Lemma 4.2, this holds for all finite $t \in T\left(A^{-j}\right)$-almost surely, as required.

We are now ready to prove the theorem.

Proof of Theorem 4.1. Let $n \in \mathbf{N}$. We must show that the following holds $\left(A^{-n}\right)$-almost surely:

$$
|M(\omega, s)-M(\omega, r)| \approx 0 \text { for all }(s, r) \in U
$$

and

$$
\|N(\omega, s)-N(\omega, r)\| / \varepsilon \approx 0 \text { for all }(s, r) \in U
$$

Let $y$ be a real number such that $0<y<1 /(2 m+4)$. Let $Q=A^{l \cdot y}$, where $l$ is the exponent such that $\delta \leq A^{-l}$ in Definition $\mathrm{A}(\mathrm{e})$. Let $\left(p^{\wedge}, X^{\wedge}\right)$ be the truncation of the original hyperfinite exchange process $(p, X)$ at $Q$. If $|X(\omega, a, s)| \leq Q$ for all $a \in A$ and all finite $s \in T$, then

$$
X(\omega, a, s)=X^{\wedge}(\omega, a, s) \text { and } p(\omega, s)=p^{\wedge}(\omega, s)
$$

for all $a \in A$ and all finite $s \in T$. By Lemma 4.11, (3) holds $\left(A^{-n}\right)$-almost surely. We have $\delta \leq A^{-l}, 1 / \varepsilon=A \cdot \delta \leq A^{1-l}$, and $\beta \leq A^{-l} \cdot \log \left(A^{1-l}\right)$, and therefore for any real $v$ between $y$ and $1 /(2 m+4)$,

$$
y+v<1 /(m+2)
$$

and

$$
Q \cdot \beta^{v} \leq A^{l \cdot(y-v)} \cdot\left(\log \left(A^{1-l}\right)\right)^{v} \approx 0 .
$$

Then $\left(A^{-n}\right)$-almost surely, (1) holds by Lemma 4.4 and (2) holds by Lemma 4.9.

\section{LAWS OF LARGE NUMBERS}

In this section we shall apply the preceding results to obtain laws of large numbers for exchange processes. 
Definition. Let $(p, X)$ be a hyperfinite exchange process. By the central path of the process, denoted by $\left(p_{1}(t), B_{1}(t)\right), t \in T$, we mean the solution of the pair of difference equations

$$
\begin{array}{cc}
p_{1}(0)=p_{0}, & \Delta p_{1}(t)=F\left(p_{1}(t), B_{1}(t)\right) \cdot \Delta t / \varepsilon, \\
B_{1}(0)=B_{0}, & \Delta B_{1}(t)=G\left(p_{1}(t), B_{1}(t)\right) \cdot \Delta t,
\end{array}
$$

where $F$ is the expected price change function and $G$ is the expected state change function. We call $p_{1}(t)$ the central price and $B_{1}(t)$ the central state at time $t$.

We wish to show that under appropriate hypotheses, the hyperfinite exchange process $(p(\omega, t), B(\omega, t))$ will be infinitely close to its central path with Loeb probability one.

Proposition 5.1. Let $(p, X)$ be a hyperfinite exchange process. Then there is a standard continuous function $b:[0, \infty) \rightarrow \mathbf{M}$ such that for all finite $t \in T$, $b(\operatorname{st}(t))$ is infinitely close to the central state at $t, B_{1}(t) \approx b(\mathbf{s t}(t))$.

Proof. By a result of Robinson [Ro], it suffices to prove that for each finite $t \in T, B_{1}(t)$ has a standard part in $\mathbf{M}$, and that whenever $t$ is finite and $t \approx u$ in $T, B_{1}(t) \approx B_{1}(u)$. (Then the function $b(\operatorname{st}(t))=\operatorname{st}\left(B_{1}(t)\right.$ ) is well defined and continuous for finite $t$.)

Let $k$ be a finite Lipschitz bound for $(p, X)$ and also a finite uniform bound for $X(\cdot, 0)$. Then $B_{1}(0) \in \mathbf{M}(J)$, where $J=2 k \cdot(k+1)$. By Corollary 3.6, there is a real constant $c$ such that $B_{1}(t) \in{ }^{*} \mathbf{M}(\exp (c \cdot t) \cdot J)$ for all $t \in T$. By Lemma 3.4, for each real $K>0$ the set $\mathbf{M}(K)$ is compact. Therefore for each finite $t \in T$ and real $K>\exp (c \cdot t) \cdot J, B_{1}(t)$ has a standard part in $\mathbf{M}(K)$ and hence in $\mathbf{M}$. Since $G$ has linear bound $k$, whenever $t<u$ in $T$ we have

$$
\begin{aligned}
\left\|B_{1}(u)-B_{1}(t)\right\| & \leq k \cdot\left(1+\sup \left(\left\|B_{1}(s)\right\|: s \leq t\right)\right) \cdot(t-u) \\
& \leq k \cdot(1+\exp (c \cdot t) \cdot J) \cdot(t-u) .
\end{aligned}
$$

Thus when $t$ is finite and $t \approx u$ in $T$,

$$
\left\|B_{1}(u)-B_{1}(t)\right\| \approx 0 \text {. }
$$

The price component $p_{1}(t)$ of the central path moves on a fast time scale where $t \in T$ is replaced by $t / \varepsilon$. It is possible for $p_{1}(t)$ to be infinite even when $t$ is finite. Starting at a finite time $s$ such that $p_{1}(s)$ is finite, we may state an analogue of Proposition 5.1.

Proposition 5.2. Let $(p, X)$ be a hyperfinite exchange process, and suppose $s \in T$ is finite and the central price $p_{1}(s)$ is finite. Then there is a standard continuous function $q_{1}:[0, \infty) \rightarrow P$ such that $p_{1}(t) \approx q_{1}(\operatorname{st}((t-s) / \varepsilon))$ whenever $s \leq t$ in $T$ and $(t-s) / \varepsilon$ is finite.

Proof. As in the preceding proof, there is a finite constant $c$ such that $\left\|B_{1}(t)\right\| \leq$ $c$ for all $t$ between $s$ and $s+1$. Then for all $p \in{ }^{*} P$ and all $t$ between $s$ and $s+1$,

$$
\left\|G\left(p, B_{1}(t)\right)\right\| \leq k \cdot(c+1) .
$$

Therefore whenever $s \leq t<u \leq s+1$,

$$
\left|p_{1}(u)-p_{1}(t)\right| \leq k \cdot(c+1) \cdot(u-t) / \varepsilon,
$$

and the result follows as before. 
Definition. Let $(p, X)$ be a starfinite exchange process and let $t_{1}$ be a positive real number or $\infty .(p, X)$ is said to be exponentially stable until $t_{1}$ if for all $t \in T$ with $\operatorname{st}(t)<t_{1}, F$ is $*$-exponentially stable for ${ }^{*} \mathbf{B}_{+}$near the central path $\left(p_{1}(t), B_{1}(t)\right)$ with positive real parameters.

If $V$ is a subset of $P \times \mathbf{B}$, we say that $F$ is exponentially stable on $V$ with parameters $b$ and $c$ if $F$ is exponentially stable for $\mathbf{B}_{+}$near every point of $V$ with parameters $b$ and $c$.

We shall be interested in the case that $(p, X)$ is exponentially stable until some positive time (real or infinite). Before proceeding to our main results, we give one case where that happens. A more general case is given in Theorem 5.7.

Proposition 5.3. Let $(p, X)$ be a hyperfinite exchange process such that the choice set $\Gamma$ is finite and the price adjustment function $f$ is standard. Suppose that $F$ is exponentially stable on a set $P \times W$, where $P$ is the price space and $W$ is an open neighborhood of $\operatorname{st}\left(B_{0}\right)$ in $\left(\mathbf{B}_{+},\|\|\right)$. Let $t_{1}$ be the exit time of the central state $B_{1}(t)$ from $W$, that is, the supremum of all real numbers st $(t)$ such that $\operatorname{st}\left(B_{1}(t)\right) \in W$. Then $t_{1}>0$ and $(p, X)$ is exponentially stable until time $t_{1}$.

Proof. If the standard part of $B_{1}(t)$ belongs to $W$, then $B_{1}(t)$ belongs to ${ }^{*} W$, and therefore $F$ is $*$-exponentially stable near $B_{1}(t)$ with the original real parameters for exponential stability on $W$.

We show that $t_{1}>0$. Let $b_{0}$ be the standard part of $B_{0}$. Since $b_{0} \in W$, there is a real $\sigma>0$ such that the closed $\sigma$-neighborhood of $b_{0}$ in $\mathbf{B}_{+}$is included in $W$. As in the proof of Proposition 5.1, there are real constants $c$ and $J$ such that for all $t \in T$ we have

$$
\left\|B_{1}(t)-b_{0}\right\| \leq k \cdot(1+\exp (c \cdot t) \cdot J) \cdot t .
$$

Therefore $\operatorname{st}\left(B_{1}(t)\right)$ is within $\sigma$ of $b_{0}$ and hence in $W$ whenever

$$
k \cdot(1+\exp (c \cdot t) \cdot J) \cdot t \leq \sigma .
$$

It follows that $t_{1}>0$.

We now come to the first main theorem.

Theorem 5.4. Let $(p, X)$ be a hyperfinite exchange process and suppose that $(p, X)$ is exponentially stable until time $t_{1}$. Then the following hold $(1 / A)$ almost surely:

(i) For all $t \in T$ such that $\mathrm{st}(t)<t_{1}$, the price is infinitely close to the central price, $p(\omega, t) \approx p_{1}(t)$.

(ii) For all $t \in T$ such that $\mathrm{st}(t)<t_{1}$, the commodity state is infinitely close to the central state, $B(\omega, t) \approx B_{1}(t)$.

Proof. Let $t_{2}$ be a positive real number less than $t_{1}$. By Lemma 4.2, it suffices to prove the result for each $t \leq t_{2}$ in $T$. Let $\omega \in \Omega$. We apply Theorem 1.17 with $X(t)=p(\omega, t)$ and $Y(t)=B(\omega, t)$ for all $t \leq t_{2}$, and $T$ equal to the set of multiples of $\Delta t$ less than $t_{2}$. We must verify that the hypotheses of Theorem 1.17 hold $(1 / A)$-almost surely. By definition, $(\bar{a}(t), \bar{b}(t))=\left(p_{1}(t), B_{1}(t)\right)$ is the central path. For each $\omega \in \Omega$, hypothesis (a) holds trivially because $p(\omega, 0)=$ $p_{1}(0)=p_{0}$ and $B(\omega, 0)=B_{1}(0)=B_{0}$. Hypothesis (c) holds because $(p, X)$ is exponentially stable until time $t_{1}$. By Theorem 4.1, hypothesis (d) holds for 
all $\omega$ in a $(1 / A)$-almost sure set $V$. Recall that the set $\mathbf{B}_{j}$ of all $b \in \mathbf{B}_{+}$such that $\|b\| \leq j$ is closed in B. By Lemmas 2.6 and 2.10, for each positive real $j>0$, the uniform Lipschitz hypothesis (b) and linear boundedness property (e) hold on the closed set $P \times \mathbf{B}_{j}$. By Corollary 3.6, there is a real $J$ such that $B_{1}(u) \in{ }^{*} \mathbf{M}(J) \subset{ }^{*} \mathbf{B}_{j}$ for all $u \leq t_{2}$. For each $j \in{ }^{*} \mathbf{N}$ let $W(j)$ be the set of all $\omega \in \Omega$ such that $B(\omega, t) \in{ }^{*} \mathbf{B}_{j}$ for all $t \leq t_{2}$. By Proposition 3.9(ii), there is a finite $c$ such that for all $j \geq c$, the internal probability of the set $W(j)$ is at least $1-1 /(A \cdot j)$. Let $j$ be finite and $j \geq c$. By Lemma 3.2, $b=b^{\wedge} j$ for all $b \in \epsilon^{*}\left(\mathbf{B}_{j} \cap \mathbf{M}\right)$. It follows from Lemmas 2.6 and 2.10 that the functions $F^{0}(p, b)=F\left(p, b^{-} j\right)$ and $G^{0}(p, b)=G\left(p, b^{-} j\right)$ are uniformly Lipschitz on ${ }^{*}(P \times \mathbf{B})$ and agree with $F$ and $G$ on ${ }^{*}\left(P \times \mathbf{B}_{j} \cap M\right)$. Let $\omega \in V \cap W(j)$ and define the functions $a^{\circ}(t), b^{\circ}(t), \bar{a}^{\circ}(t), \bar{b}^{\circ}(t)$ for $t \in T$ by

$$
\begin{gathered}
a^{\circ}(0)=p(0), \quad \Delta a^{\circ}(t)=F^{\circ}\left(a^{\circ}(t), b^{\circ}(t)\right) \cdot \Delta t / \varepsilon+\Delta M(\omega, t), \\
b^{\circ}(0)=B(0), \quad \Delta b^{\circ}(t)=G^{\circ}\left(a^{\circ}(t), b^{\circ}(t)\right) \cdot \Delta t+\Delta N(\omega, t), \\
\bar{a}^{\circ}(0)=p(0), \quad \Delta \bar{a}^{\circ}(t)=F^{\circ}\left(\bar{a}^{\circ}(t), \bar{b}^{\circ}(t)\right) \cdot \Delta t / \varepsilon, \\
\bar{b}^{\circ}(0)=B(0), \quad \Delta \bar{b}^{\circ}(t)=G^{\circ}\left(\bar{a}^{\circ}(t), \bar{b}^{\circ}(t)\right) \cdot \Delta t .
\end{gathered}
$$

All the hypotheses of Theorem 1.17 hold for the above difference equations, so by Theorem 1.17 we have

$$
a^{\circ}(t) \approx \bar{a}^{\circ}(t) \text { and } b^{\circ}(t) \approx \bar{b}^{0}(t)
$$

for all $t \leq t_{2}$ in $T$. Since $\bar{B}_{1}(t) \in{ }^{*} \mathbf{B}_{j}$ and $B(\omega, t) \in{ }^{*} \mathbf{B}_{j}$ for all $t \leq t_{2}$, $p(\omega, 0)=p_{1}(0)=p_{0}$ and $B(\omega, 0)=B_{1}(0)=B_{0}$, we see by internal induction that for all $t \leq t_{2}$ in $T$,

$$
\begin{aligned}
p_{1}(t) & =\bar{a}^{\circ}(t), & & B_{1}(t)=\bar{b}^{\circ}(t), \\
p(\omega, t) & =a^{\circ}(t), & & B(\omega, t)=b^{\circ}(t) .
\end{aligned}
$$

Thus

$$
p(\omega, t) \approx p_{1}(t), \quad B(\omega, t) \approx B_{1}(t)
$$

for all $\omega$ in the set $V \cap W(j)$ and all $t \geq t_{2}$ in $T$. Since $V$ is $(1 / A)$-almost sure and each $W(j)$ has internal probability at least $1-1 /(A \cdot j)$, the union of the sets $V \cap W(j), c \leq j \in \mathbf{N}$, is $(1 / A)$-almost sure.

Here is a translation of Theorem 5.4 into standard terms.

Theorem 5.5 (standard form of Theorem 5.4). For all positive integers $d, m$, and $j$ and positive real numbers $k_{1}, k_{2}, k_{3}, k_{4}, l, t_{1}$, and $\alpha$ such that $l \in$ $(0,1)$ there exists a positive real naumber $A_{0}$ such that the following holds: Let $(p, X)$ be a finite exchange process with parameters $f, g, \Gamma, \delta$, and $\Delta t$. Assume that

(a) The price space $P$ and commodity space $C$ have dimensions $d$ and $m$.

(b) The initial price $p(0)$ and allocation $X(a, 0)$ are uniformly bounded by $k_{1}$.

(c) $(p, X)$ is Lipschitz with bound $k_{2}$.

(d) The number of agents $A$ is at least $A_{0}$.

(e) $\delta=A^{-l}$. 
(f) $F$ is exponentially stable with parameters $k_{3}, k_{4}$ near $\left(p_{1}(t), B_{1}(t)\right)$ for all $t \leq t_{1}$ in $T$.

Then with probability at least $1-1 /(A \cdot j)$, for all $t \leq t_{1}$ in $T$ we have

$$
\left|p(\omega, t)-p_{1}(t)\right| \leq \alpha \text { and }\left\|B(\omega, t)-B_{1}(t)\right\| \leq \alpha .
$$

The notion of an $\left(A^{-j}\right)$-almost sure set was introduced in $\S 4$ in order to prove the next theorem.

Theorem 5.6. Let $(p, X)$ be a hyperfinite exchange process and suppose that $(p, X)$ is exponentially stable until $t_{1}$. Then for all $t \in T$ with $\operatorname{st}(t)<t_{1}$, the central path of $(p, X)$ is infinitely close to the expected value of $(p(\omega, t)$, $B(\omega, t))$,

$$
p_{1}(t) \approx E_{\Omega}[p(\cdot, t)] \text { and } B_{1}(t) \approx E_{\Omega}[B(\omega, t)] .
$$

In fact, whenever $\operatorname{st}(t)<t_{1}$,

$$
E_{\Omega}\left[\max \left(\left|p(\cdot, u)-p_{1}(u)\right|: u \leq t\right)\right] \approx 0
$$

and

$$
E_{\Omega}\left[\max \left(\left\|B(\cdot, u)-B_{1}(u)\right\|: u \leq t\right)\right] \approx 0 .
$$

Proof. We first observe that since $B(\omega, t)$ is $\mathbf{F}_{t}$-measurable, the expected value of $B(\omega, t)-B_{1}(t)$ is a $*$-finite sum, and by the triangle inequality we have

$$
\begin{aligned}
\left\|E_{\Omega}\left[B(\cdot, t)-B_{1}(t)\right]\right\| & \leq E_{\Omega}\left[\left\|B(\cdot, t)-B_{1}(t)\right\|\right] \\
& \leq E_{\Omega}\left[\max \left(\left\|B(\cdot, u)-B_{1}(u)\right\|: u \leq t\right)\right] .
\end{aligned}
$$

Let $t \in T$ and $\operatorname{st}(t) \leq t_{1}$. Define $N(\omega)=\max (n(\omega, a, t): a \in A)$. We have $\max (\operatorname{abs}(X(\omega, a, u)): a \in A$ and $u \leq t) \leq(k+1)^{N(\omega)+1}$

and therefore

$$
\max (\|B(\omega, u)\|: u \leq t) \leq(k+1)^{N(\omega)+1} .
$$

Also, since the price adjustment function has linear bound $k$ on $C$,

$$
\max (|p(\omega, u)|: u \leq t) \leq(k+1)^{N(\omega)+2} / \varepsilon .
$$

By Corollary 3.6 there is a real constant $c_{1}$ such that for all $u \leq t,\left\|B_{1}(u)\right\| \leq$ $c_{1}$. Thus

$$
\max \left(\left\|B(\omega, u)-B_{1}(u)\right\|: u \leq t\right) \leq c_{1}+(k+1)^{N(\omega)+1} .
$$

Moreover,

$$
\max \left(\left|p_{1}(u)\right|: u \leq t\right) \leq(k+1) \cdot\left(c_{1}+1\right) \cdot t / \varepsilon
$$

so

$$
\max \left(\left|p(\omega, u)-p_{1}(u)\right|: u \leq t\right) \leq c_{2} / \varepsilon+(k+1)^{N(\omega)+2} / \varepsilon
$$

where $c_{2}=(k+1) \cdot\left(c_{1}+1\right) \cdot t$. Let $L$ be the greatest element of ${ }^{*} \mathbf{N}$ such that $(k+1)^{L+1}<A$. By the proof of Lemma 4.11, $N(\omega) \leq L\left(A^{-j}\right)$-almost surely for each finite $j$. On the set $\{\omega: N(\omega)<L\}$, we have

$$
\max \left(\left\|B(\omega, u)-B_{1}(u)\right\|: u \leq t\right) \leq 2 A
$$

and

$$
\max \left(\left|p(\omega, u)-p_{1}(u)\right|: u \leq t\right) \leq 2 A \cdot(k+1) / \varepsilon=2 A^{2-l} \cdot(k+1)
$$


It follows from Theorem 5.4 that the set $\{\omega: N(\omega) \leq L\}$ makes an infinitesimal contribution to each of the expected values

$$
E_{\Omega}\left[\max \left(\left\|B(\omega, u)-B_{1}(u)\right\|: u \leq t\right)\right]
$$

and

$$
E_{\Omega}\left[\max \left(\left|p(\omega, u)-p_{1}(u)\right|: u \leq t\right)\right] .
$$

By Lemma 3.8, for each $n$ we have $P_{\Omega}[N(\cdot)=n] \leq A \cdot t^{n} / n$ !. Therefore for each $n \geq L$, the set $\{\omega: N(\omega)=n\}$ makes a contribution of at most

$$
2 A \cdot(k+1) \cdot(t \cdot(k+1))^{n} / n ! \leq 2 \cdot\left(t \cdot(k+1)^{2}\right)^{n+1} / n !
$$

to the expected value $E_{\Omega}\left[\max \left(\left\|B(\omega, u)-B_{1}(u)\right\|: u \leq t\right)\right]$. Summing over $n$, we see that the set $\{\omega: N(\omega) \geq L\}$ makes a contribution of at most the infinitesimal quantity $4 \cdot\left(t \cdot(k+1)^{2}\right)^{L+1} / L$ ! to the expected value. This shows that

$$
E_{\Omega}\left[\max \left(\left\|B(\omega, u)-B_{1}(u)\right\|: u \leq t\right)\right] \approx 0 .
$$

By a similar argument,

$$
E_{\Omega}\left[\max \left(\left|p(\omega, u)-p_{1}(u)\right|: u \leq t\right)\right] \approx 0 .
$$

In the next result we obtain equations for the expected values of $p(\omega, t)$ and $B(\omega, t)$ in terms of the functions $F$ and $G$. For this purpose we shall take the price adjustment and excess demand functions $f$ and $g$ to be standard (or, in the formulation of Theorem 5.5, we take $f$ and $g$ to be fixed before choosing the parameters $A_{0}, A_{1}$, and $\delta_{0}$ ).

Theorem 5.7. Let $(p, X)$ be a hyperfinite exchange process. Suppose the choice set $\Gamma$ is finite, and the functions $f$ and $g$ are standard. Let $q_{0}=\operatorname{st}\left(p_{0}\right)$ and $b_{0}=\operatorname{st}\left(B_{0}\right)$ be the standard parts of the initial price and commodity state. Assume that

(i) The solution $q(s)$ of the differential equation $q(0)=q_{0}, q^{\prime}(s)=$ $F\left(q(s), b_{0}\right)$ approaches a limit $q(\infty)$ as $s \rightarrow \infty$.

(ii) $H$ is a uniformly Lipschitz function from an open subset $W$ of $\left(\mathbf{B}_{+},\|\|\right)$ into $P$ such that $b_{0} \in W, H\left(b_{0}\right)=q(\infty)$, and $F(H(b), b)=0$ for all $b \in W$.

(iii) $F$ is exponentially stable on an open set containing the graph of $H^{-1}$ in the space $P \times\left(\mathbf{B}_{+},\|\|\right)$.

Let $b(t), t<t_{1}$, be the solution of the standard initial value problem

$$
b(0)=b_{0}, \quad b^{\prime}(t)=G(H(b(t)), b(t)),
$$

where $t_{1}$ is the supremum of all $t$ for which the solution exists.

Then $t_{1}>0,(p, X)$ is exponentially stable until time $t_{1}$, and the central path $\left(p_{1}(t), B_{1}(t)\right)$ satisfies

$p_{1}(t) \approx q(t / \varepsilon)$ for all $t \approx 0$ in $T$;

$p_{1}(t) \approx H(b(\mathbf{s t}(t)))$ for all $t \in T$ such that $0<\operatorname{st}(t)<t_{1}$;

$B_{1}(t) \approx b(\operatorname{st}(t))$ for all $t \in T$ such that $\operatorname{st}(t)<t_{1}$.

Proof. Since $X(\cdot, 0)$ has a finite uniform bound, $b_{0}=\mathbf{s t}\left(B_{0}\right)$ belongs to $\mathbf{M}(J)$ for some finite $J$. By Theorem 3.7, the solution $b(t)$ exists uniquely as long as $b(t)$ belongs to $W$. Since $W$ is open, this happens up to some time $t_{1}>0$. 
It follows from Proposition 5.1 that whenever $t \approx 0$ in $T, B_{1}(t) \approx b_{0}$, and hence by the Lipschitz property of $F$,

$$
F\left(p_{1}(t), B_{1}(t)\right) \approx F\left(p_{1}(t), b_{0}\right) .
$$

Putting $s=t / \varepsilon$ and comparing the difference equation for $p_{1}(t)$ and the differential equation in (i) for $q(s)$, we see that for real $s$ the function $q_{1}(s)=$ $\operatorname{st}\left(p_{1}(s \cdot \varepsilon)\right)$ is a solution of the differential equation in (i). Since the solution is unique (because $F$ is locally Lipschitz) and approaches $q(\infty)$ as $s$ approaches $\infty$, we have $p_{1}(t) \approx q(t / \varepsilon)$ for $t \approx 0$ in $T$.

In particular, whenever $t \approx 0$ and $t / \varepsilon$ is infinite, $p_{1}(t) \approx q(\infty)$. Let $t_{0}, t_{2} \in$ $T$ be such that $0=\operatorname{st}\left(t_{0}\right)<\operatorname{st}\left(t_{2}\right)<t_{1}$ and $t_{0} / \varepsilon$ is infinite. By (iii), $F$ is exponentially stable on an open set $V$ containing the graph of $H^{-1}$ with positive real parameters $a$ and $a^{\prime}$. Since $b$ and $H$ are continuous, the set

$$
S=\left\{(H(b(u)), b(u)): 0 \leq u \leq \operatorname{st}\left(t_{2}\right)\right\}
$$

is a compact subset of $V$. Choose a positive real number $\alpha$ small enough so that every point in $P \times \mathbf{B}_{+}$within $\alpha$ of some point in $S$ in both the $P$ and $\mathbf{B}_{+}$components belongs to $V$.

Let $B_{2}(u)$ be given by the difference equation

$$
B_{2}\left(t_{0}\right)=b_{0}, \quad \Delta B_{2}(u)=G\left(H\left(B_{2}(u)\right), B_{2}(u)\right) \Delta t .
$$

Let $p_{2}(u)=H\left(B_{2}(u)\right)$. The pair of functions $p_{2}(u)$ and $B_{2}(u)$ satisfies the pair of difference equations

$$
\begin{gathered}
\Delta p_{2}(u)=F\left(p_{2}(u), B_{2}(u)\right) \cdot \Delta t / \varepsilon+\Delta M_{2}(u), \\
\Delta B_{2}(u)=G\left(p_{2}(u), B_{2}(u)\right) \cdot \Delta t,
\end{gathered}
$$

where

$$
F\left(p_{2}(u), B_{2}(u)\right)=0, \quad \Delta M_{2}(u)=H\left(B_{2}(u+\Delta t)\right)-H\left(B_{2}(u)\right) .
$$

$B_{2}(t)$ satisfies the difference equation corresponding to the differential equation $b^{\prime}(t)=G(H(b(t)), b(t))$. Also, $B_{2}\left(t_{0}\right)=b_{0}=b(0) \approx b\left(t_{0}\right)$.

Since $G$ and $H$ are locally Lipschitz and $\Delta t \approx 0$, we have

$$
b\left(\operatorname{st}(t) \approx b(t) \approx B_{2}(t) \text { and } H(b(\operatorname{st}(t))) \approx H(b(t)) \approx p_{2}(t)\right.
$$

for all $t \in T$ between $t_{0}$ and $t_{2}$. Since $b$ and $H$ are continuous, for each $t \in T$ between $t_{0}$ and $t_{2}$ the standard part of $\left(p_{2}(t), B_{2}(t)\right)$ belongs to $S$, so any point within $\alpha / 2$ of $\left(p_{2}(t), B_{2}(t)\right)$ belongs to ${ }^{*} V$. By transfer, $F$ is *-exponentially stable near any point within $\alpha / 2$ of $\left(p_{2}(t), B_{2}(t)\right)$ with parameters $a$ and $a^{\prime}$. Thus to prove the theorem it suffices to prove that $p_{1}(t) \approx p_{2}(t)$ and $B_{1}(t) \approx B_{2}(t)$ for all $t \in T$ between $t_{0}$ and $t_{2}$.

We now show that whenever $t_{0} \leq t \leq t_{2}$ and

$$
p_{1}(u) \text { and } B_{1}(u) \text { are within } \alpha / 2 \text { of } p_{2}(u) \text { and } B_{2}(u)
$$

for all $t_{0} \leq u<t$, we have

$$
p_{1}(u) \approx p_{2}(u) \text { and } B_{1}(u) \approx B_{2}(u)
$$

for all $t_{0} \leq u<t$. Let $t_{0} \leq t \leq t_{2}$ and suppose (2) holds for all $t_{0} \leq u<t$. Then $F$ is *-exponentially stable near $\left(p_{1}(u), B_{1}(u)\right)$ for $t_{0} \leq u<t$ with parameters $a$ and $a^{\prime}$. 
We wish to apply Theorem 1.17 to the pairs of functions $\left(p_{1}, B_{1}\right)$ and $\left(p_{2}, B_{2}\right)$ for $t_{0} \leq u<t$. Since $H$ has Lipschitz bound $k$,

$$
\begin{aligned}
\left|\Delta M_{2}(u)\right| & \leq k \cdot\left\|B_{2}(u+\Delta t)-B_{2}(u)\right\| \\
& \leq k \cdot\left\|G\left(p_{2}(u), B_{2}(u)\right)\right\| \cdot \Delta t \leq k \cdot(k+2) \cdot \Delta t .
\end{aligned}
$$

Thus whenever $|s-u| \leq \varepsilon$ we have $M_{2}(s) \approx M_{2}(u)$. By Theorem 1.17, (3) holds for all $t_{0} \leq u<t$.

We now use internal induction to show that (2) holds for all $t_{0} \leq u \leq t_{2}$. We have

$$
p_{1}\left(t_{0}\right) \approx q(\infty)=H\left(b_{0}\right)=p_{2}\left(t_{0}\right), \quad B_{1}\left(t_{0}\right) \approx b_{0}=B_{2}\left(t_{0}\right),
$$

so (2) holds for $u=t_{0}$. Suppose $t_{0}<t \leq t_{2}$ and (2) holds for all $t_{0} \leq u<t$. Then by the preceding paragraph, (3) holds for all $t_{0} \leq u<t$. Since all the terms of the difference equations are infinitesimal, we have

$$
p_{1}(t) \approx p_{1}(t-\Delta t) \approx p_{2}(t-\Delta t) \approx p_{2}(t)
$$

and

$$
B_{1}(t) \approx B_{1}(t-\Delta t) \approx B_{2}(t-\Delta t) \approx B_{2}(t) .
$$

Hence (2) holds when $u=t$. This completes the induction.

It follows that (3) holds for all $t_{0} \leq u<t_{2}$. This completes the proof of the theorem.

Remark. Both Proposition 5.3 and Theorem 5.7 can be improved slightly by replacing $\mathbf{B}_{+}$by the union of $\mathbf{M}(J)$ for finite $J$.

By using Theorem 4.10 instead of Theorem 4.1, we obtain "bounded forms" of Theorems 5.4-5.7, with Definition $\mathrm{A}(\mathrm{e})$ replaced by the condition that $\delta, \varepsilon$, and $\beta$ are infinitesimal and the functions $f$ and $g$ have finite uniform bounds. Here is the bounded form of Theorem 5.4.

Theorem 5.8 (bounded form of Theorem 5.4). Let $(p, X)$ be a hyperfinite exchange process but with condition (e) of Definition A replaced by :

$\left(\mathrm{e}^{\prime}\right) \delta, \varepsilon=1 /(A \cdot \delta)$, and $\beta=\delta \cdot \log (A \cdot \delta)$ are infinitesimal, and the ranges of $f$ and $g$ have a finite uniform bound.

Suppose that $(p, X)$ is exponentially stable until $t_{1}$. Then for some positive real $q$ the following holds $\exp \left(-\beta^{-q}\right)$-almost surely:

$$
p(\omega, t) \approx p_{1}(t) \text { and } B(\omega, t) \approx B_{1}(t)
$$

for all $t \in T$ such that $\operatorname{st}(t)<t_{1}$.

The results of this paper can readily be generalized to the case that the price adjustment function $f$ and excess demand function $g$ depend on the time $t$ and directly on the argument $b$ in $\mathbf{B}_{+}$as well as on $p \in P$ and $x \in C$. Thus

$$
\begin{aligned}
& f: \Gamma \times P \times C \times \mathbf{B}_{+} \times[0, \infty) \rightarrow P, \\
& g: \Gamma \times P \times C \times \mathbf{B}_{+} \times[0, \infty) \rightarrow C .
\end{aligned}
$$

We shall assume that $f$ and $g$ are uniformly Lipschitz in all arguments and linearly bounded on $C$.

For $p \in P, b \in \mathbf{B}_{+}, t \in[0, \infty)$, and $\varphi \in \mathbf{L}$, the auxiliary functions $\bar{f}(p, b, t): C \rightarrow P$ and $\bar{g}(p, b, t, \varphi): C \rightarrow R$ are defined by

$$
\begin{gathered}
\bar{f}(p, b, t)(x)=E_{\Gamma}[f(\cdot, p, x, b, t)] \\
\bar{g}(p, b, t, \varphi)(x)=E_{\Gamma}[\varphi(x+g(\cdot, p, x, b, t))]-\varphi(x) .
\end{gathered}
$$


Then the functions

$$
F: P \times \mathbf{B}_{+} \times[0, \infty) \rightarrow P, \quad G: P \times \mathbf{B}_{+} \times[0, \infty) \rightarrow \mathbf{B}
$$

are defined by

$$
F(p, b, t)=b(\bar{f}(p, b, t)), \quad G(p, b, t)(\varphi)=b(\bar{g}(p, b, t, \varphi)) .
$$

The central path $\left(p_{1}(t), B_{1}(t)\right)$ is defined by the difference equations

$$
\begin{gathered}
p_{1}(0)=p_{0}, \quad \Delta p_{1}(t)=F\left(p_{1}(t), B_{1}(t), t\right) \cdot \Delta t / \varepsilon, \\
B_{1}(0)=B_{0}, \quad \Delta B_{1}(t)=G\left(p_{1}(t), B_{1}(t), t\right) \cdot \Delta t .
\end{gathered}
$$

In this setting, we say that the hyperfinite exchange process $(p, X)$ is exponentially stable until time $t_{1}$ if for all $t \in T$ with $\operatorname{st}(t)<t_{1}$, the function $F_{t}: P \times \mathbf{B}_{+} \rightarrow P$ defined by $F_{t}(p, b)=F(p, b, t)$ is $*$-exponentially stable near $\left(p_{1}(t), B_{1}(t)\right)$ with positive real parameters.

Theorems 5.4-5.8 all hold in this more general setting.

In the special case that the price adjustment function $f$ is identically zero, there is no longer a fast and slow component and the price adjustment parameter $\delta$ plays no role. In this case the result reduces to a theorem of Oelschlager [O] on "weakly interacting particle systems," and when the function $g$ has a finite range, to an earlier theorem of Kurtz [Ku].

Our results can also be generalized to the case where the elements of the choice set $\Gamma$ are chosen with probabilities which depend on $p$ and $x$, rather than with uniform probability. Let $h$ be a function $h: \Gamma \times P \times C \rightarrow[0,1]$ which is uniformly Lipschitz on $P \times C$ and such that for each $p \in P$ and $x \in C$,

$$
\sum_{\gamma \in \Gamma} h(\gamma, p, x)=1 .
$$

We may consider a modified finite exchange process in which the sample space $\Omega$ has the probability measure such that for each $\omega \in \Omega, a \in A, \gamma \in \Gamma$, and $t \in T$, the probability that $\omega(t+\Delta t)=(a, \gamma)$ given $\omega \mid t$ is equal to $h(\gamma, p(\omega, t), X(\omega, a, t)) \cdot \Delta t$. The function $F$ and $G$ are defined as before but with expected values taken with respect to the new measure on $\Omega$. One can readily verify that $F$ and $G$ still have the properties proved in $\S 2$, with appropriate changes in the bounds given in Lemmas 2.4 and 2.8. The remaining results are proved as before.

A special case which arises naturally in many examples from economics is the case where the probability of choosing an agent $a \in A$ depends on the price $p(\omega, t)$ and commodity vector $X(\omega, a, t)$. This case can be obtained by putting a special element $\circ$ into $\Gamma$ such that $f(\circ, p, x)=0$ and $g(\circ, p, x)=$ 0 . When $\omega_{\Gamma}=0, p$ and $X$ do not change. This is equivalent to the process where the element $\circ$ is left out of $\Gamma$ but each agent $a$ is chosen with probability

$$
1-h(\circ, p(\omega, t), X(\omega, a, t)) \cdot \Delta t \text {. }
$$

\section{REFERENCES}

[AFHL] S. Albeverio, J. E. Fenstad, R. Hoegh-Krohn, and T. Lindstrom, Nonstandard methods in stochastic analysis and mathematical physics, Academic Press, 1986.

[Au] R. J. Aumann, Market with a continuum of traders, Econometrica 32 (1964), 39-50. 
[B] S. N. Bernstein, Probability theory, Moscow, 1946. (Russian)

[BR] D. J. Brown and A. Robinson, Nonstandard exchange economies, Econometrica 43 (1975), 41-55.

[CL] E. A. Coddington and N. Levinson, Theory of ordinary differential equations, McGraw-Hill, 1955.

[EK] S. Ethier and T. Kurtz, Markov processes, Wiley, 1986.

[G] Douglas Gale, Bargaining and competition. I, II, Econometrica 54 (1986), 785-818.

[Ha] F. Hahn, Stability, Chapter 16, Handbook of Mathematical Economics, vol. II (K. J. Arrow and M. D. Intriligator, eds.), North-Holland, 1982.

[Hi] W. Hildenbrand, Core and equilibria of a large economy, Princeton Univ. Press, 1974.

[K1] H. J. Keisler, An infinitesimal approach to stochastic analysis, Mem. Amer. Math. Soc. No. 297 (1984).

[K2] _ A price adjustment model with infinitesimal traders, Models of Economic Dynamics (H. Sonnenschein, ed.), Springer-Verlag, 1986, pp. 69-87.

[K3] _ Decentralized markets with fast price adjustment (to appear).

[Ku] T. Kurtz, Strong approximation theorems for density dependent Markov chains, Stochastic Process. Appl. 6 (1978), 223-240.

[L] T. M. Liggett, Interacting particle systems, Springer-Verlag, 1985.

[O] K. Oelschlager, A martingale approach to the law of large numbers for weakly interacting stochastic processes, Ann. Probab. 12 (1984), 458-479.

[OM] Robert E. O’Malley, Jr., Introduction to singular perturbations, Academic Press, 1974.

[Ra] R. Radner, Equilibrium under uncertainty, Chapter 20, Handbook of Mathematical Economics, vol. II (K. J. Arrow and M. D. Intriligator, eds.), North-Holland, 1982.

[Ro] A. Robinson, Nonstandard analysis, North-Holland, 1966.

[Sm] S. Smale, A convergent process of price adjustment and global Newton methods, J. Math. Econom. 3 (1976), 1-14.

[SB] K. D. Stroyan and J. M. Bayod, Foundations of infinitesimal stochastic analysis, NorthHolland, 1986.

[VDB] Imme van den Berg, Nonstandard asymptotic analysis, Lecture Notes in Math., vol. 1249, Springer-Verlag, 1987.

[W] W. Wasow, Asymptotic expansions for ordinary differential equations, Wiley, 1965.

Department of Mathematics, University of Wisconsin, Madison, Wisconsin 53706

E-mail address: keisler@math.wisc.edu 